\title{
Occupational Safety Review Of High Technology Facilities
}

L. C. Cadwallader

January 2005

Idaho National Engineering and Environmental Laboratory Bechtel BWXT Idaho, LLC 
INEEL/EXT-05-02616

\title{
OCCUPATIONAL SAFETY REVIEW OF HIGH TECHNOLOGY FACILITIES
}

\author{
L. C. Cadwallader
}

Published January 2005

Idaho National Engineering and Environmental Laboratory

Bechtel BWXT Idaho, LLC

PO Box 1625

Idaho Falls, Idaho 83415-3860

Prepared for the

U.S. Department of Energy, Idaho Operations Office

Under Contract No. DE-AC07-99ID13727 


\begin{abstract}
This report contains reviews of operating experiences, selected accident events, and industrial safety performance indicators that document the performance of the major US DOE magnetic fusion experiments and particle accelerators. These data are useful to form a basis for the occupational safety level at matured research facilities with known sets of safety rules and regulations. Some of the issues discussed are radiation safety, electromagnetic energy exposure events, and some of the more widespread issues of working at height, equipment fires, confined space work, electrical work, and other industrial hazards. Nuclear power plant industrial safety data are also included for comparison.
\end{abstract}




\section{SUMMARY}

An issue of interest with the International Thermonuclear Experimental Reactor (ITER) design is the safety of the personnel who will operate and maintain the facility. Since the ITER machine is much larger than existing experiments and will handle higher power levels and input energies than present tokamaks, there is a concern that the personnel will be at higher risk than the presently accepted levels of risk seen in the existing fusion experiments. The existing US tokamaks have not had any personnel fatalities, and severe occupational accidents have also been rare. Similar operating experiences with the largest US particle accelerators have had fatalities during construction, but have experienced any fatalities in operation. The accelerators have had a few severe occupational accidents. The accelerators and fusion experiments have had occupational injury rates that were somewhat higher than other DOE research and development facilities. In recent years, the fission power plants have had lower occupational accident/injury rates than either fusion or the accelerators, but the power plant accident severity may be greater, and the power plants have had some occupational fatalities while fusion and accelerator operations have not. These data, along with safety performance statistics, have been documented in this report. The case history descriptions presented here are useful to understand the types of occupational events that have occurred and the energy sources involved in the events. These data will support occupational safety analysis of ITER operations, especially a room-by-room analysis of energies and hazards that ITER workers will experience during plant operation and maintenance. The safety performance statistics presented here highlight areas for improvement in occupational safety at fusion and accelerator facilities; ITER can use these data to form more robust occupational safety programs. The statistics also support setting occupational safety goals for ITER operation. Due to its size and power levels, ITER is envisioned to be greater than existing fusion experiments but less than a nuclear fission power plant, and safety goals can be set accordingly. 


\section{CONTENTS}

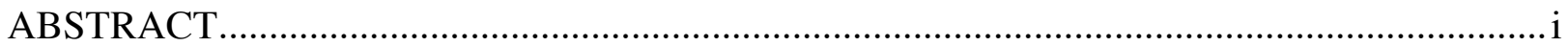

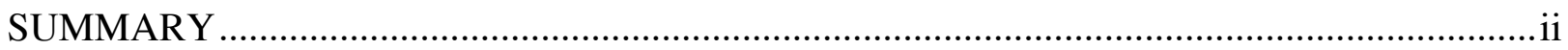

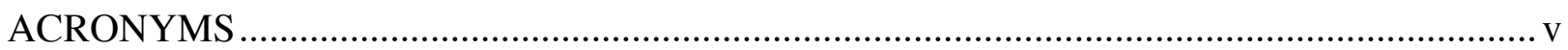

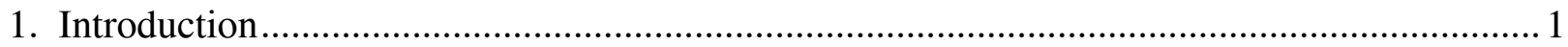

2. Magnetic Fusion Experiment Occupational Safety .................................................... 3

2.1 Princeton Plasma Physics Laboratory .................................................................. 3

2.2 Other Fusion Machines .................................................................................... 16

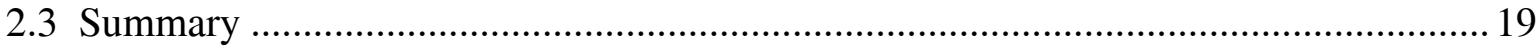

3. Particle Accelerator Occupational Safety ................................................................... 25

3.1 Stanford Linear Accelerator Center ..................................................................... 25

3.2 Fermi National Accelerator Laboratory .............................................................. 44

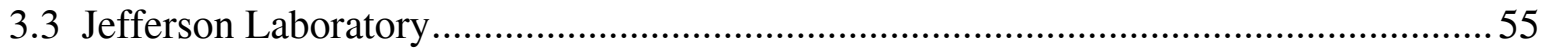

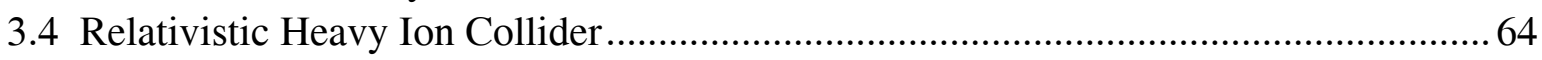

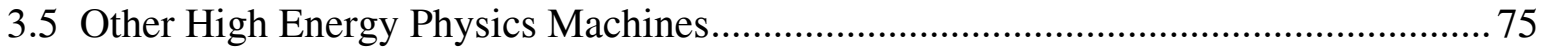

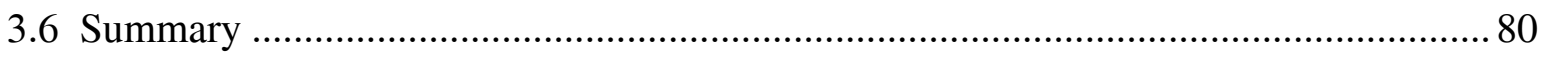

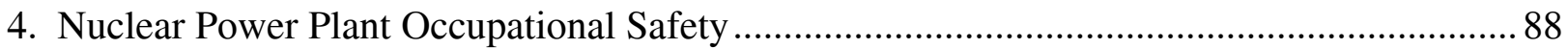

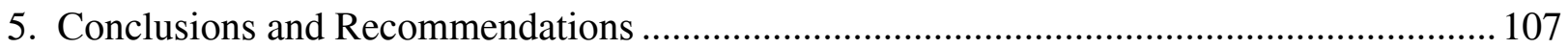




\section{TABLES}

1. Occupational Safety Data for US Industrial Workers............................................... 21

2. Occupational Safety Data for the Electric, Gas, and Sanitary Services in the US. ............. 22

3. Operational Events at Particle Accelerators. ............................................................. 76

4. Early Values of Occupational Safety Rates for the Nuclear Fission Industry .....................89

5. Recent Power Reactor Events of Industrial Safety Concern ........................................ 98

\section{FIGURES}

1. Plots of PPPL Industrial Safety Performance from 1993-2003 ........................................5

2. PPPL Industrial Injury Distributions from 1981-2003 …....................................... 6

3. Plots of SLAC Industrial Safety Performance from 1993-2003 ..................................... 27

4. SLAC Industrial Injury Distributions from 1981-2003 ............................................. 28

5. Plots of FNAL Industrial Safety Performance from 1993-2003 .................................... 45

6. FNAL Industrial Injury Distributions from 1981-2003 ............................................ 46

7. Plots of CEBAF Industrial Safety Performance from 1993-2003 ..................................56

8. CEBAF Industrial Injury Distributions from 1988-2003 ….......................................57

9. Plots of All DOE Research Industrial Safety Performance from 1993-2003 _....................81 


\section{ACRONYMS}

AGS Alternating Gradient Synchrotron at Brookhaven National Laboratory

ATR AGS-to-RHIC transfer line

BLS Bureau of Labor Statistics, US Department of Labor

BWR boiling water reactor

CAIRS Computerized Accident/Incident Reporting System

CEBAF Continuous Electron Beam Accelerator Facility

D\&D decontamination and decommissioning

D-T deuterium-tritium

DOE Department of Energy

ES\&H Environment, Safety and Health

FNAL Fermi National Accelerator Laboratory

HP health physics

IAEA International Atomic Energy Agency

IDLH Immediately Dangerous to Life or Health

INPO Institute of Nuclear Power Operations

ITER International Thermonuclear Experimental Reactor

Jlab Thomas Jefferson National Accelerator Laboratory (formerly CEBAF)

LER Low Energy Ring

LER Licensee Event Report

LLNL Lawrence Livermore National Laboratory

LN2 liquid nitrogen

LPG liquefied petroleum gas

LWC Lost Work Case

LWD Lost Work Day

MG motor-generator

MCC motor control center

NCSX National Compact Stellarator Experiment

NRC Nuclear Regulatory Commission

NSTX National Spherical Torus Experiment 


\begin{tabular}{|c|c|}
\hline $\mathrm{ODH}$ & oxygen deficiency hazard \\
\hline ORPS & Occurrence Reporting and Processing System \\
\hline OSHA & Occupational Safety and Health Administration \\
\hline PBX & Princeton Beta Experiment \\
\hline PBX-M & Princeton Beta Experiment-Modification \\
\hline PDX & Poloidal Divertor Experiment \\
\hline PEL & permissible exposure limit \\
\hline PEP & Positron Electron Project at SLAC \\
\hline PLT & Princeton Large Torus \\
\hline PPE & personal protective equipment \\
\hline PPPL & Princeton Plasma Physics Laboratory \\
\hline PWR & pressurized water reactor \\
\hline rem & roentgen-equivalent-man \\
\hline RF & radiofrequency \\
\hline RHIC & Relativistic Heavy Ion Collider at Brookhaven National Laboratory \\
\hline SLAC & Stanford Linear Accelerator Center \\
\hline SPEAR & Stanford Positron-Electron Asymmetric Ring \\
\hline SSRL & Stanford Synchrotron Radiation Laboratory \\
\hline $\mathrm{Sv}$ & Sievert \\
\hline TFTR & Tokamak Fusion Test Reactor \\
\hline TRC & Total Recordable Cases \\
\hline $\mathrm{V}$ & volts \\
\hline VESDA & very early smoke detection apparatus \\
\hline WANO & World Association of Nuclear Operators \\
\hline
\end{tabular}




\section{OCCUPATIONAL SAFETY REVIEW OF HIGH TECHNOLOGY FACILITIES}

\section{$\mathbf{1 . 0}$ INTRODUCTION}

The International Thermonuclear Experiment Reactor (ITER) project proposes to build a large tokamak experiment that will burn a deuterium-tritium plasma. The machine will be larger and will handle more power and fuel than existing experiments. Consequently, the personnel safety concerns are also greater with this machine than with past machines. The ITER team has examined occupational safety and set limits for exposure to hazardous energies and materials, including ionizing radiation, non-ionizing radiation, and beryllium. The ITER team has also identified other industrial hazards associated with ITER, including high voltage electricity, cryogens, confined spaces, fires, chemical hazards, mechanical hazards, rotating machinery, and lifting equipment (e.g., cranes) (IAEA, 2002). This report contains an occupational safety review of US Department of Energy (DOE) research facilities that are similar to ITER - mainly fusion experiments and particle accelerators. The experiences of these facilities should be indicative of the hazards that ITER is likely to encounter during operation. The results of this review can be used to support the ITER occupational safety assessment, which the host country is expected to request as part of the licensing process. The US DOE directs that fusion facility workers shall be protected such that the risks to which they are exposed at a fusion facility are no greater than those to which they would be exposed at a comparable industrial facility (DOE, 1996). The ITER host country is anticipated to have a similar requirement for the safety of workers.

This report also contains information about US nuclear fission power plant occupational safety. Power plants are included because ITER is a much larger experiment than the currently existing tokamaks in the world; ITER is approaching a small power plant in size and scale. ITER cooling systems operate at moderate pressures and temperatures ( 4 $\mathrm{MPa}$ and $100^{\circ} \mathrm{C}$ ), at parameters greater than existing fusion machines but somewhat less than fission power plant parameters. In that respect, nuclear power plant occupational safety should provide an upper bound of the ITER safety envelope. Another fact to consider is that ITER will have frequent maintenance sessions for both refurbishment of the machine and systems and for equipment/diagnostics upgrades or modifications. This is expected, as it is the nature of experimental facilities. Power plants operate continuously over long times and have short duration maintenance outages with few upgrades or modifications; consequently the power plant workers have more time at risk during plant operations and generally shorter, more hurried maintenance sessions. Such factors must be taken into account for occupational safety. 
Chapter 1 references

DOE, 1996. Safety of Magnetic Fusion Facilities: Requirements, DOE-STD-6002-96, US Department of Energy (May 1996).

IAEA, 2002. ITER Technical Basis, ITER EDA Documentation Series No. 27, International Atomic Energy Agency, Vienna (2002) chapter 5. 


\subsection{MAGNETIC FUSION EXPERIMENT OCCUPATIONAL SAFETY}

This chapter presents descriptions of occupational accidents in US fusion experiments. Some statistics are also presented regarding the rates of injuries and types of injuries sustained.

\subsection{Princeton Plasma Physics Laboratory}

The major US fusion site that has operated the most experiments is also the best known site - the Princeton Plasma Physics Laboratory (PPPL) in Princeton, New Jersey. This site also has safety data available from the DOE. As a single purpose site, the PPPL data are easily retrieved from the appropriate DOE data bases. PPPL has about 400 total employees and an annual budget of approximately $\$ 55 \mathrm{M}$. PPPL was the first fusion laboratory in the US; in the 1950's and 1960's, stellarators were used to study fusion. In the 1970's, tokamaks became the leading fusion research machines. There have been several tokamaks of interest, including the Princeton Large Torus (PLT), which was operated in the 1970's and 1980's, and the Tokamak Fusion Test Reactor (TFTR), which was operated in the 1980's and 1990's. The PLT studied radiofrequency (RF) heating and current drive, neutral beam injection heating, and other aspects of tokamak physics. The PLT is regarded as a direct predecessor to the TFTR. The Poloidal Divertor Experiment (PDX) was a medium sized experiment in the 1970's to study the effectiveness of poloidal magnetic divertors to control impurities in fusion plasmas. The PDX was modified and became the Princeton Beta Experiment (PBX). The PBX investigated advanced tokamak regimes, such as indented plasmas with high beta factors, and the second stability regime. The PBX was later modified and became PBX-M, to investigate higher plasma pressure operation. Only the TFTR used tritium (T) fuel; the other machines were fueled with hydrogen, hydrogen-deuterium, or deuterium (D). Using the smaller hydrogen isotopes was safety conservative since they are not radioactive, and their reactions do not emit the very high energy neutrons like D-T reactions. Low neutron fluence over machine life kept the radiation fields manageable.

Newer experiments are the National Spherical Torus Experiment (NSTX) (Neumeyer, 1999) and the National Compact Stellarator Experiment (NCSX) (Nelson, 2003). A time line of some of the major experiments at PPPL (Tanner, 1982) is:

Princeton Large Torus (PLT), R = 1.32 m, a = 0.4 m, I = 1.6 MA (Hosea, 1985)

Construction 1972-1975

First discharge December 20, 1975

Poloidal Divertor Experiment (PDX), $\mathrm{R}=1.4 \mathrm{~m}, \mathrm{a}=0.45 \mathrm{~m}, \mathrm{I}=500 \mathrm{kA}$

Construction 1975-1978

First discharge November 29, 1978; converted to "PBX" by 1985 
Princeton Beta Experiment (PBX), R = $1.4 \mathrm{~m}, \mathrm{a}=0.45 \mathrm{~m}, \mathrm{I}=500 \mathrm{kA}$ (Bol, 1985)

Construction PDX conversion to PBX began in mid-1983 (Bol, 1983),

PBX operated 1985-1989, and the conversion to

PBX-Modification began in 1987

First discharge $\quad$ PBX operated in 1985; the PBX-M operated 1989-1994.

Tokamak Fusion Test Reactor (TFTR), $\mathrm{R}=2.65 \mathrm{~m}, \mathrm{a}=1.1 \mathrm{~m}, \mathrm{I}=2.5 \mathrm{MA}$

Construction 1977-1982

First discharge December 24, 1982 (Machalek, 1983)

National Spherical Torus Experiment (NSTX), $\mathrm{R}=0.85 \mathrm{~m}, \mathrm{a}=0.67, \mathrm{I}=1.0 \mathrm{MA}$

Construction 1997-1999

First discharge February 12, 1999 (Neumeyer, 2000)

National Compact Stellarator Experiment (NCSX), $\mathrm{R}=1.4 \mathrm{~m}$, average $\mathrm{a}=0.3$, plasma beta $=4 \%, \mathrm{I} \sim 50 \mathrm{kA}$ (see ncsx.pppl.gov)

Construction final design review May 2004, fabrication in late 2004, magnet construction 2005-06, NCSX construction 2006-08

First discharge scheduled for late 2008

It is also notable that the TFTR machine ceased operation in April 1997 and after a period of safe shutdown in 1997-1999, was decommissioned between late 1999 to 2002 (Perry, 1999; Raftopoulos, 2002). The staff dealt with many commonplace hazards, including power tool injuries, falls, lacerations, oxygen deficient spaces, electrical hazards, and hoisting and rigging hazards (Raftopoulos, 2002). The lead oxide on shielding bricks to be decommissioned and the beryllium dust chemical contamination from machine operations both posed hazards until the particulates were removed with strippable coatings (Lumia, 2002; Lumia, 2003).

The industrial safety performance of the PPPL staff over the last decade has been obtained from the DOE Computerized Accident/Incident Reporting System (CAIRS) and plotted in Figures 1 and 2. Overall, the data in Figure 1 show a positive trend of decreasing the lost work days, which means PPPL is working on reducing the number and severity of accidents. This is positive since the PPPL values are higher than the overall R\&D values presented later this report. The PPPL peak values in 1994, 1997, and 1999-2002 are explained by the activities under way at the lab during those years. In 1994, the PBX-M was shut down and was placed into safe, cold shut down mode. In 1997, the TFTR ceased its D-T operations and entered a safe shut down mode, which entailed a great deal of work with all the tokamak subsystems. In 1999, the NSTX machine began operations, but, more importantly, the TFTR began decontamination and decommissioning (D\&D). That was a significant task, demanding large efforts from the staff in tasks that they were not as familiar with as operations. The PLT and PBX-M machines were dismantled and removed from their test cell building in the same time 


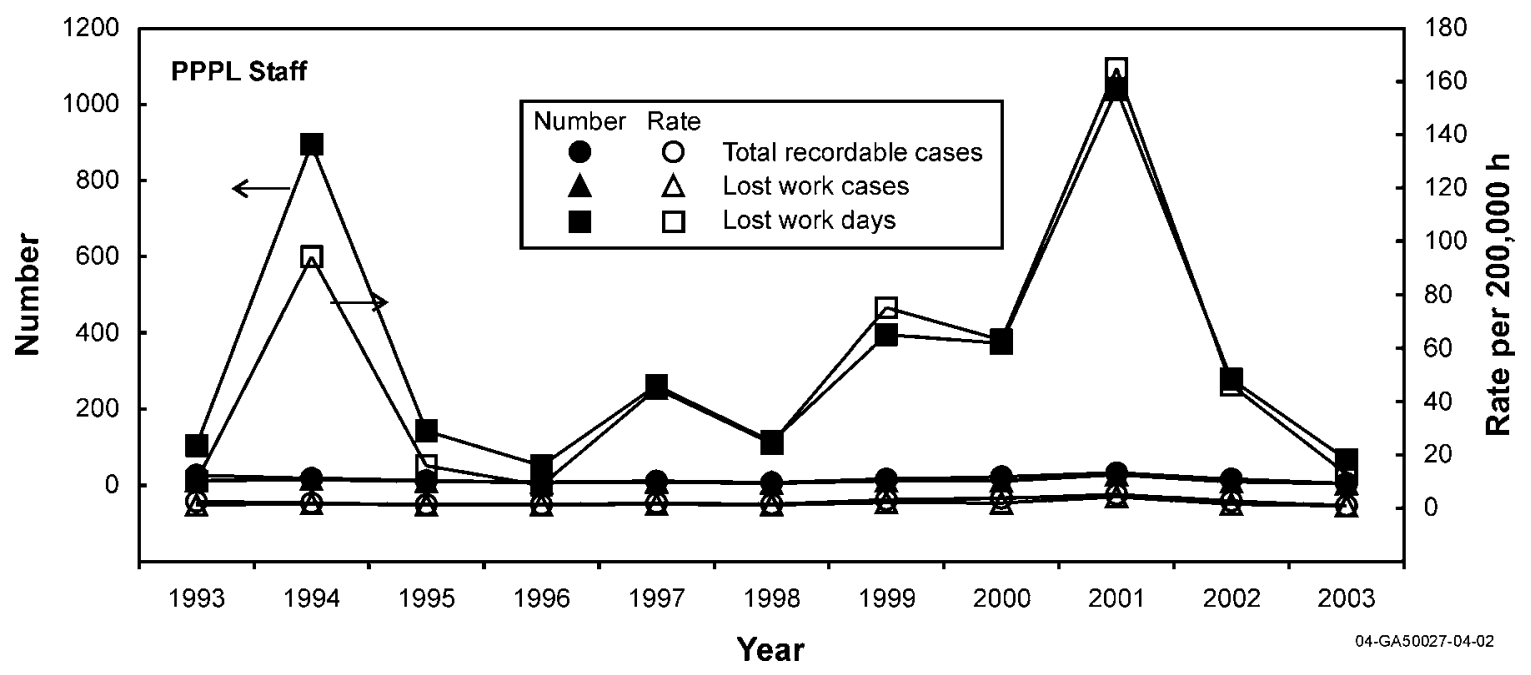

note: The DOE data have shown that no staff or other workers have suffered a work-related fatality while working at PPPL.

Figure 1. Plots of PPPL Industrial Safety Performance from 1993-2003.

period as TFTR D\&D. Their removal made way for the NCSX device, which is currently under construction. The NCSX will use neutral beam, vacuum pumping, and water cooling systems from the PBX-M, and power supplies from the TFTR. The occupational injury distribution results are presented in Figure 2. The first chart shows that lacerations are frequent. The 'other injuries' category includes punctures, dislocations, cumulative trauma, dermatitis, eye injuries, insect bites, hernia, injuries "not otherwise classified", and other types of injuries that individually were small in number but summed together comprise almost a third of all reported injuries. The second chart in Figure 2 shows that finger injuries are the most common, which is to be expected with the large amount of hands-on work involved with tokamak maintenance. The third chart shows that technicians have the highest number of injuries incurred in their work, this is because the technicians are continually closest to the tokamak systems and perform the majority of the hands-on tasks. The data plotted in Figure 1 can be compared to the research facility totals that will be presented later in this report (see Figure 9). The charts from Figure 2 can be compared to the similar charts given for accelerator facilities. Note that PPPL did not report construction contractor or services subcontractor injuries. Some sites report their construction contractor and services (electricians, technicians, custodians, etc.) injuries, especially when the contracts involve significant amounts of manpower.

There have been several published events of industrial safety concern at PPPL. The most noteworthy events are discussed here. On September 12, 1970, employees were conducting a scheduled power outage. After completing scheduled maintenance work, an attempt was made to restore normal power. Immediately after closing the main $138 \mathrm{kV}$ 


\section{PPPL Staff Injuries by Type}

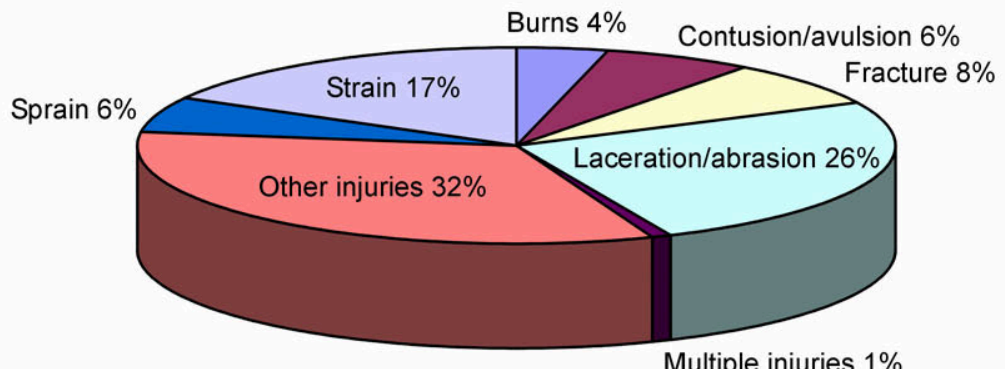

Multiple injuries $1 \%$

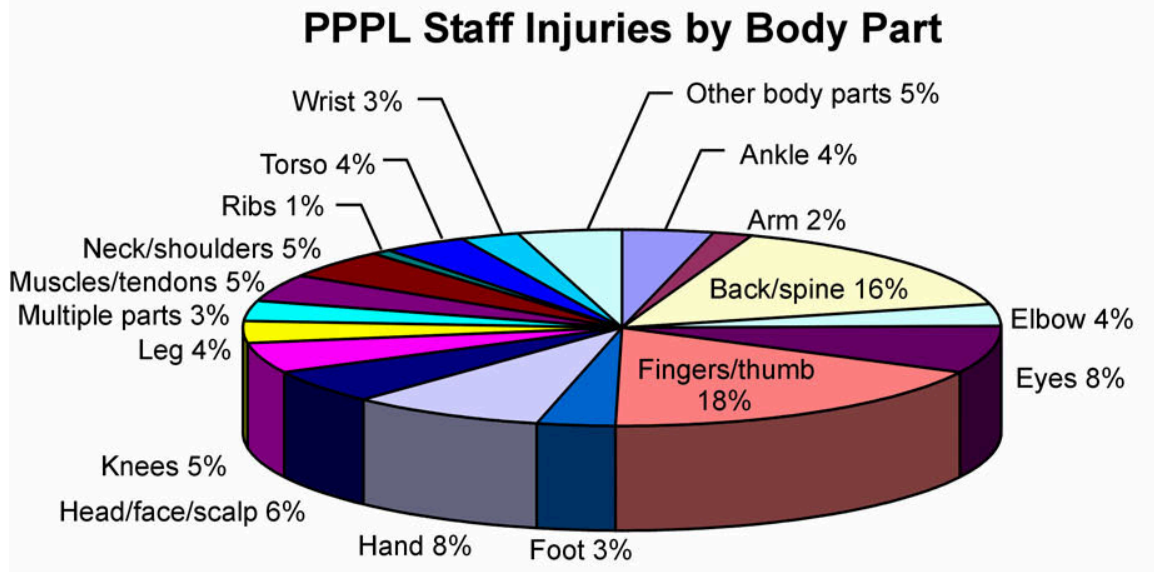

\section{PPPL Staff Injuries by Occupation}

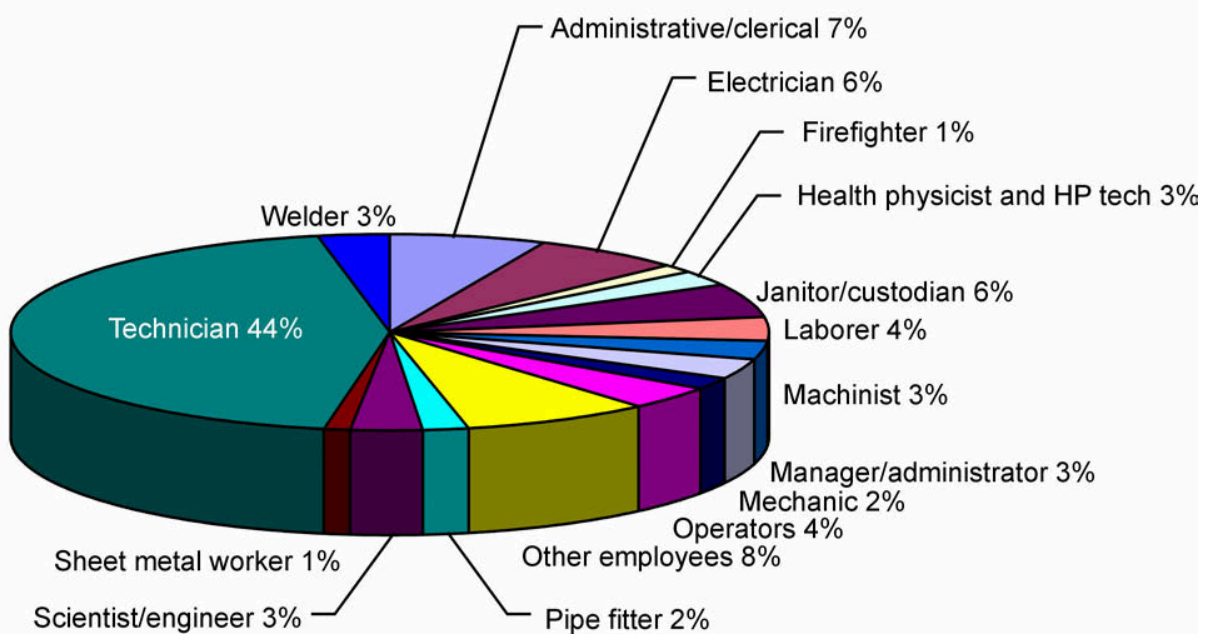

Figure 2. PPPL Industrial Injury Distributions from 1981-2003. 
circuit breaker, a short circuit occurred on the secondary side of the power system in the 4160-V circuit breaker cubicles. The fault was not cleared by the protective relays tripping the $138 \mathrm{kV}$ breaker, but rather remained on for approximately 30 seconds before the breaker was manually opened. The electrical flashover in the 4160-V switchgear cubicle caused the resulting fire and damage to three units of switchgear. This damage could probably have been less if the $138 \mathrm{kV}$ circuit breaker on the incoming service line had properly tripped open to clear the fault condition. Roughly half of the $\$ 51 \mathrm{k}$ loss was due to replacement costs of the three switchgear units. The remaining cost was labor to remove damaged parts and install the replacement parts. There were no personnel injuries in this event (WASH, 1975). Princeton documentation also describes this event in more detail (Tanner, 1982). The fire was described as the most damaging fire in the first 30 years of laboratory operations. An eyewitness recounted the following events:

"After a few hours of outage for routine maintenance, difficulty was experienced in closing the automatic circuit breaker XB-1, and after about two hours of troubleshooting they decided to attempt closure using manual activation of the closure mechanism. They followed the manufacturer's instructions to manually close the breaker. The oil pressure in the breaker was raised to $2,800 \mathrm{psi}$, which is adequate for closure. The breaker closed. Then one of the employees saw that the ground resistors of the two neutrals on the step down transformer were smoking. He called to the other workers to manually open the breaker. When he looked back at the ground resistors, they were in flames. He ran to a telephone and notified a guard to call the municipal fire department. He returned to help the other employees fight the fire with hand-held fire extinguishers. They limited the fire to one extensively damaged breaker and heavy damage to the adjacent two breakers. The PPPL fire truck was deployed and relieved the employees using hand-held extinguishers with the truck-fed extinguishing equipment. When the municipal fire department arrived, the flames were extinguished and they provided cooling to the fire damaged equipment to prevent re-ignition." \{Fortunately, there were no personnel injuries and the personnel were trained in the proper use of fire extinguishers.

The DOE Occurrence Reporting and Processing System (ORPS) lists 197 off-normal events for PPPL between 1990 and 2003. Some of these events are environmental release issues, noncompliances, security issues, equipment leaks and failures that did not endanger personnel, several events of receipt of counterfeit and substandard replacement parts, and a few events of on-site digging that severed underground telephone, electrical, sewer, and natural gas lines. While the natural gas line breach events did pose an obvious combustion hazard, there were no adverse consequences from the gas leaks. The ORPS events related to industrial safety are described below. These descriptions provide the details of energy sources that harmed workers and the progression of accident events. 
On September 10, 1990, a maintenance subcontractor attempted to lift the 16-ton stator of a motor-generator (MG) set with 75-ton crane. Unknown to the subcontract workers, two hidden 1.5-inch bolts were still fastened. These two bolts were broken during the lift. The direct cause of this incident was the failure of subcontractor personnel to recognize and remove two bolts holding the upper half of the field yoke assembly. The subcontractor personnel made assumptions that all the bolting was removed. Effective investigation during the lift was not performed when the field yoke assembly did not react the way it was expected as the lift proceeded. PPPL personnel failed to research all of the available documentation and hence failed to locate and use an existing drawing which showed the bolt pattern. The root cause involved the failure of the subcontractor foreman to perform a detailed investigation of all possible mounting bolts prior to initiating the lift (ORPS CH-PA-PPPL-PPPL-1990-0001).

On March 28, 1991, two personnel performing maintenance on the D-Site MG crane traversed the room by walking on a 25 to $30 \mathrm{~cm}$ building frame I-beam about $18 \mathrm{~m}$ above the ground. They were not wearing safety harnesses. No one was injured. The workers were ordered to don safety harnesses. Several of the issues associated with this occurrence were identified in the Tiger Team review earlier this year and are in the process of being addressed (ORPS CH-PA-PPPL-PPPL-1991-0028).

On the morning of April 12, 1991, a work assignment was started which would involve entry into TFTR Neutral Beam line \#4, which is a confined space. During the work authorization process, two individuals were found to be in the confined space for which the 'Confined Space Entry Work Permit' had expired. The entry safety checklist prerequisites (air monitoring, ventilation, electrical isolation, safety watch) were implemented and followed; however, the job had not yet been checked by the Environment, Safety \& Health (ES\&H) Office, nor the permit authorized by the ES\&H Office prior to entry. At the time the incident took place, efforts were underway to obtain the necessary authorization; however, the employees assumed an expired permit, still posted at the entry point, was the new authorized permit. A contributing cause for this occurrence involved the fact that no one had been specifically assigned the responsibility to remove permits once they expire. In this case, two expired permits (covering two sequential time periods) were posted at the entry point. A permit can only remain valid for 30 days. The employees had requested a new permit, and were following the confined space rules for air monitoring, ventilation, electrical isolation, safety watch, etc. However, the ES\&H Office had not yet issued a permit before the workers entered the space (ORPS CH-PA-PPPL-PPPL-1991-1002).

On May 1, 1991, a PPPL employee noticed a manhole grate with a water hose protruding from the manhole. He did not see a posted Confined Space Entry Permit. Upon further investigation, he found that a maintenance technician had entered the well (manhole access) to make a water connection. (The maintenance technician was performing cleanup work on the exterior of the QA office trailers at the time). A confined Space Entry Permit had not been obtained. This is a violation of the PPPL procedure on Confined Space Entry. The maintenance technician was counseled on the need for a confined space entry permit. The technician had worked at PPPL for over 29 years and had not 
needed such a permit in the past. In January 1991, PPPL identified and labeled over 500 confined spaces at the facility (ORPS CH-PA-PPPL-PPPL-1991-1004).

On May 16, 1991, two PPPL employees were allowed to enter the TFTR Test Cell without a film badge dosimeter. This is a violation of the established rules for entry into the Test Cell. At no time were these individuals in a radiation area. The two individuals were counseled on the requirement for wearing dosimeters in the TFTR Test Cell and the TFTR Test Cell Basement (ORPS CH-PA-PPPL-PPPL-1991-1006).

On December 5, 1991, the water pump motor 806 (a 600 horsepower, 4160 Volt motor in the D site pump house) failed electrically by a turn-to-turn short circuit in the motor coils. A ground fault was created which caused a power outage in portions of PPPL's "B" and "C" sites. The extent of the power outage was determined. Upon the report of the pump motor failure, the Emergency Services Unit responded to the Pump House. An active fire condition did not exist. TFTR and PBX-M terminated operations, and the "C" and "D" site motor generators were brought to a halt. A small fire was discovered and immediately extinguished in a vacuum pump motor. Electrical service to PPPL was restored within approximately 60 minutes. The on-line spare pump was brought into service after power was restored. TFTR experienced a 4-hour delay in operations, and PBX-M was not restarted; operations re-scheduled the week as a maintenance week (ORPS CH-PA-PPPL-PPPL-1991-1035).

On January 21 and 22, 1992, a technician was assigned to cut lead blocks for a diagnostic on the PBX-M Device. Prior to the work, the PPPL Industrial Hygienist was contacted for advice. At his advice, the employee was outfitted with a belt mounted air sampling unit during the cutting operation. On February 21, 1992, the lab results from the sampling unit were received and found to be 139 and 169 micro-grams per cubic meter, while the permissible limit for personnel exposure is 50 micro-grams per cubic meter. The work was stopped and the employee had blood work done to determine if his lead uptake was significant. The test results showed he did not have elevated lead concentration in his blood. The exact cause of apparent exposure is not known. It may have been via the lead oxide loosened during the handling of the lead or it may have been only a sampling error caused by the employee adjusting the position of the sampling tube wearing the gloves that had been handling the lead (ORPS CH-PA-PPPL-PPPL-19920003). A related event occurred at the Microwave Tokamak Experiment in 1992 when shielding bricks were moved and restacked; the exposure is discussed later in this chapter.

On March 9, 1992, an employee caught his foot on table leg while rising from the desk in his office. The employee fell on his hip. X-rays indicated a broken femur. The office was found to be a safe environment and was not cluttered. This was considered to be a highly unusual, unique event (ORPS CH-PA-PPPL-PPPL-1992-0007).

On April 6, 1992, a subcontractor employee who was performing maintenance on the PPPL C-Site Cooling Tower sustained an injury due to a fall from a ladder. The worker fell with the ladder as the ladder fell from the scaffolding near the tower. The victim was 
transported by ambulance to the hospital. The work crew had abandoned the original scaffolding as being too dangerous and they had designed their own scaffolding, but this scaffolding did not have rails or toeboards, and used inadequate ladders (ORPS CH-PAPPPL-PPPL-1992-0012).

On August 14, 1992, a halon system discharge occurred when a subcontractor was working in the computer Tape Vault. It is believed that the sensor was tripped while the technician was replacing ceiling tiles, possibly due to a defective sensor. Investigation indicates that the thermal detector that senses a fire and activates the halon system was not properly installed (it was placed above the ceiling tiles instead of protruding through it). It also appears that the detector was defective. In addition, investigation revealed that the sensor was inappropriately relocated to a new position above the ceiling tile at some time in the past as part of an unrelated maintenance activity. About $8.6 \mathrm{~kg}$ of halon were discharged. The subcontractor apparently was not injured in this event (ORPS CH-PAPPPL-PPPL-1992-0023).

On October 20, 1992, at approximately 1550 hours, it was discovered that an individual had apparently exceeded the PPPL TFTR radiation exposure limit of $25 \mathrm{mrem} / \mathrm{day}$ $(0.25 \mathrm{mSv} /$ day $)$. The individual operating the crane had received a dose of $39 \mathrm{mrem}$ $(0.39 \mathrm{mSv})$, as determined by the individual's digital dosimeter, within a two hour time period. The individual receiving the dose was assisting a team of technicians in the installation of the alpha charge exchange analyzer. The other technicians involved in the work task did not exceed the administrative limit. The direct cause for this event involved failure to follow established requirements for access to the Test Cell with respect to calculating stay times and monitoring of the self-reading dosimeter. A contributing cause was determined to be the content of the previously administered radiation safety training (for the $25 \mathrm{mrem} /$ day $[0.25 \mathrm{mSv} /$ day] administrative limit). Although this requirement had apparently been discussed in training sessions, it was not formally included in the course material. Another contributing cause was determined to be the fact that a radiation survey was not performed of the crane area to determine the dose rate in the elevated portion of the Test Cell. The root cause was the inadequacy of the administrative procedure used to control access to the Test Cell following high power operations (ORPS CH-PA-PPPL-PPPL-1992-0028).

On December 7, 1992, an electrical deficiency caused a minor fire in a computer connection cabinet. Four circuit boards burned. An electrical duplex outlet was found to be defective. Other units in 150 offices were inspected and modified as needed. When the Walker box (a junction box) was swiveled to allow outlets to be accessible, the 120 Volt ac hot wire insulation was damaged and contacted the Walker box wall, putting the case at 120 Vac. This voltage was applied to the personal computer and to the communication electronics in the room, resulting in the fire. No one was injured in this fire (ORPS CH-PA-PPPL-PPPL-1992-0032).

On January 15, 1993, a subcontract employee was assisting the vendor in a test of TFTR tritium seal dampers. The individual attempted to close fire damper within a duct and became entrapped in the inspection port due to its size $(20 \times 30 \mathrm{~cm})$. Confined space 
procedures were violated. The PPPL Emergency Service Unit (ESU) responded. The victim freed himself from the duct work before the ESU arrived. ESU personnel evaluated the victim. The individual refused medical treatment. Work inside of the ducts was stopped until the event was evaluated. The individual had entered the confined space without a hazard evaluation and a confined space permit by the Industrial Hygienist. There was a lack of adequate supervision of the individual performing the work. His supervisor did not acknowledge safety responsibility for the individual who entered the confined space (ORPS CH-PA-PPPL-PPPL-1993-0002).

On Friday, April 2, 1993, a custodial staff member stepped over a yellow safety rope with a danger sign in the PPPL C-Site Motor Generator Building and approached to within approximately $30 \mathrm{~cm}$ of a $50-\mathrm{kV}$ hipot machine. Hipotting of ground sticks was in progress at the time, although the machine was not energized at the time of the occurrence. (note: hipotting is a 'high potential difference' voltage test, a type of electrical insulation test, performed every 2 years as per 29CFR1910.269) Test operations personnel intercepted the custodian and escorted him out of the area. The custodian was counseled on the hazards of ignoring the safety barriers (ORPS CH-PAPPPL-PPPL-1993-0011).

On April 20, 1993, during work to activate a new modular building at the PPPL C-Site, a subcontract technician entered a confined space [a telephone manhole] and was not confined space trained. The individual entered momentarily (after explicitly being told not to enter the confined space by a PPPL Industrial Hygienist) to retrieve a roll of fish line used to pull cable for utility installation. The subcontract technician and the Facilities Engineering Representative were strongly reprimanded on the failure to obey instructions (ORPS CH-PA-PPPL-PPPL-1993-0017).

On June 30, 1993, a subcontractor employee of a construction company was discovered working without fall protection on the RF stair tower. The subcontractor was completing outstanding construction work on the stair tower. The work consisted of placing flashing on the edge of the top surface. Upon discovery that the individual was working without fall protection, the work activity was terminated. General terms and conditions of the contract call for compliance with OSHA regulations and PPPL safety standards. In this case the employee willingly chose to disregard both as an act of convenience to complete his portion of the work scope. Apparently, he rationalized the potential risk of working without fall protection. Obviously, it is a safety requirement that should not be rationalized away. The contract agency was immediately notified that the employee was in violation of PPPL policy and OSHA regulations and he was further requested to immediately and permanently remove the employee from the project site. The contract agency complied with this request. No one was injured although the potential for a serious accident existed (ORPS CH-PA-PPPL-PPPL-1993-0022).

On August 11,1997 a 480 Volt three-phase electrical line was cut during mechanical cutting operations of a $35 \mathrm{~cm}$ concrete floor in D-Site Mock-Up Building East. The floor cut was being made to install a jib crane foundation. Although a Digging Permit had been obtained for the cutting operation, an obvious discrepancy existed, because the 
drawing utilized to issue the permit indicated no conduit traversing the area. The alternating current power group did not participate in the permitting process due to limited available staff. On his own cognition the operator wore rubber boots, leather gloves, and had insulating handles on the saw. When the building lights went out during the cut, he stopped work. No injuries occurred during this incident, although the potential for injury was present (ORPS CH-PA-PPPL-PPPL-1997-0002).

On August 28, 1997, work to disassemble a glovebox was in progress. A piece of copper pipe with a valve and a cap, which was determined to be clean (external) by a tritium technician was carried out of the Tritium Area by another technician to a shop for rework. The copper pipe was not surveyed by a health physics technician. The tritium technician took the copper pipe to a welding area and soldered a coupling to the pipe. Next, he took the pipe to the vacuum prep area to test leak tightness. After that he took the pipe to the neutral beam machine shop where he performed other work. Then he was called away and he left the pipe in the machine shop. The health physics technicians stated that they had requested the technicians to "hold all line breaks" until they could finish other tasks in other parts of the facility and return to the Tritium Area. The tritium technicians determined that they could continue with some tasks despite the lack of health physics technicians. When the next shift began, the new health physics technician noted that there was tritium contamination in the area due to the open end of the copper pipe. The pipe end was covered and 'elephant trunked' while the pipe piece was located. Subsequent surveys have determined that the piece of pipe that was carried out of the area was contaminated with tritium (15.5 million $\mathrm{dpm} / 100 \mathrm{~cm}^{2}$ internal). No personnel and no areas were surface contaminated, but the leak detector coupling was contaminated. The health physics manager evaluated the radiological data for the event and determined that there may have been some personnel contamination, particularly on the technician who soldered the pipe. Bio-assays performed on the individuals involved in this incident indicated a maximum individual dose of 1.1 mrem (11 microSv), a minimal worker exposure (ORPS CH-PA-PPPL-PPPL-1997-0004).

On July 31, 1998, four PPPL personnel were making preparations for the removal of a mechanical pump from the Neutral beam Vacuum Pumping system in the TFTR Test Cell Basement. TFTR was in a caretaking mode at that time, and the pump was being removed permanently. Due to the configuration of the pump and hardware system, the workers found it necessary to reposition the horizontal section of flexible corrugated hose that led to the blower pump, still attached a tee, to allow them access to attach blank flanges. In the process of repositioning the corrugated hose in the exhaust line of the pump, an estimated $50 \mathrm{~cm}^{3}$ of contaminated oil dripped out from a horizontal section of the exhaust line where oil had accumulated. The oil dripped onto the pump and an oil pan below the pump. The tritium vapors from the contaminated oil set off the room's area monitor, at which time the pump and exhaust line openings were blanked off. The workers then proceeded to clean up the oil until instructed by a health physics representative to go to the Safety Check Area. The workers were swiped by health physics personnel and it was determined that they were contaminated at levels between approximately 5,900 and $11,300 \mathrm{dpm} / 100 \mathrm{~cm}^{2}$. The basement was put into full purge mode, the area was decontaminated and then secured. The workers were 
decontaminated. Workers must be retrained on the response required when a tritium alarm is activated (ORPS CH-PA-PPPL-PPPL-1998-0006).

On January 28, 1999, a maintenance worker entered a water utility pit to complete work that had started the day before. This entry into a confined space was done with a confined space permit, which had expired the previous day. In addition, the worker entered the confined space without using the full body harness and retrieval gear specified by the permit because the Emergency Services Unit was unable to locate this gear the day before. The task to be performed was simple and brief, plugging in a cord, and the worker believed that the process of finding the appropriate personnel and equipment, the necessity of entering the space to have enough clearance to don the harness, then donning the gear, was more time consuming than the task itself. He did follow the atmosphere monitoring procedure before entering the pit. He was counseled on following the rules and all maintenance workers were informed that if they have disputes with the rules that they need to discuss them with their supervisor for resolution instead of violating the rules (ORPS CH-PA-PPPL-PPPL-1999-0001).

On June 22, 2000, a health physics technician was identified as having exceeded the contamination level of $10,000 \mathrm{dpm} / 100 \mathrm{~cm}^{2}$. The level was identified through routine smears required by the Radiological Work Permit. The contamination was identified on the technician's face. This incident was caused by the technician accidentally touching his face after touching the equipment that was being worked on. The technician was wearing personal protective equipment (PPE), but was not wearing the appropriate PPE to contact the equipment. The manager conducted a review of the work practices associated with the incident. Upon review of the data from the smears on all personnel, along with the bioassay results, and interviews of the three technicians working in the containment tent, it is believed that this incident was isolated and caused by a direct action of the technician touching the equipment being worked on and then inadvertently touching his face. At the time the technician was wearing PPE, but not the correct PPE that would have allowed him to contact the equipment directly. There was no health consequence of this short duration contamination exposure. The technicians were reminded to use caution when doffing their PPE to avoid skin contamination (ORPS CHPA-PPPL-PPPL-2000-0005).

On August 15, 2000, three employees were assigned a task to cut a 20-cm diameter pipe in the TFTR building. After trying two other methods, the employees selected a hydraulically operated cutting tool (a pincer type tool) in an attempt to cut the pipe. The pipe was too large to fit completely between the cutting jaws of the tool. The workers then tried to cut the pipe by working inward through the pipe. They used the tips of the tool jaws to compress the pipe, enabling the cutter to reach further onto the pipe. During this process, the cutting tool was continuously twisting due to side loading of the blades. During the second cut of this pipe with the cutting tool, the continual side-loading of the blade caused the tip of the blade to fracture, propelling it approximately $6 \mathrm{~m}$ across the room until it impacted a tool chest. The cause of this incident was that the individuals operated the equipment without the required practice or hands-on experience with the equipment. In addition, closer supervision should have been provided to ensure that the 
employees did not try to operate equipment with which they had limited experience or in a manner inconsistent with manufacturer's recommendations. No one was injured by the cutter blade (ORPS CH-PA-PPPL-PPPL-2000-0007).

On August 22, 2000, two technicians, and one HP technician, were in the process of removing RF antenna feedthroughs in the TFTR Test Cell area. At one hour into the job, routine nasal and facial samples were taken from each worker. The results of the analysis determined that the results for one technician was $11,696 \mathrm{dpm}$ per $100 \mathrm{~cm}^{2}$ by nasal sample. All other smears were significantly less than $10,000 \mathrm{dpm}$ per $100 \mathrm{~cm}^{2}$ for all other workers in the area. The workers in the area were consistently observed by an HP Technician and were in full compliance with the approved radiation work permit. The job was immediately stopped. The employee was decontaminated, and bio-assay samples were collected. The cause of this incident could not be positively verified. It appears that all requirements were met, and the radiological work permit was in compliance with expected environment in the area. The logical conclusion for this incident appears to be that the individual inadvertently touched his nose when removing the mask; however, this could not be validated. There was no health consequence due to this contamination event (ORPS CH-PA-PPPL-PPPL-2000-0008).

On December 5, 2001, while placing a concrete-filled TFTR Vacuum Vessel Segment in a Type "A" shipping container, the lid (approximately $681 \mathrm{~kg}$ ), which was leaning against the container, was pushed over when the Vacuum Vessel Segment came in contact with the lid. A health physics technician stopped to watch the lowering operation; then when the vessel segment appeared to be in the shipping container, the technician moved into the immediate area. The lid then fell in the vicinity of the health physics technician. Workers yelled and he quickly moved to evade the lid. The lid struck the toroidal field coil holding/disassembly fixture. The job was stopped and the lid was laid on the ground. A few days earlier, the container had been placed in the test cell and the lid removed. There was not enough open floor space available to lay the lid on the floor. The bottom of the lid was fastened to prevent it from sliding out, but the top of the lid was not fastened. When the vessel segment was being lowered into the container, the segment had to be repositioned. During repositioning, the segment contacted the lid, which resulted in the lid falling over. No one was injured in this event. Procedures were changed to better control the lifts (ORPS CH-PA-PPPL-PPPL-2001-0006).

On March 26, 2002, at approximately 0920 hours, the Laboratory Site Protection Division received a call, via the Emergency Notification System, that a subcontractor had been injured while working in a trench. Upon arrival the Emergency Services Unit determined that the individual was pinned, and the PPPL Mutual Aid Agreement was activated. The local fire company and ALS medical unit responded to the scene, and the county trench rescue team was automatically placed on stand-by, but was not required to respond to the incident. Upon arrival at the scene it was determined that a dense mass composed of clay, stone, soil, and sand had broken loose from the sidewall of the trench and fell onto the lower leg of a subcontractor working in the $>1.5 \mathrm{~m}$ deep trench. The weight of the mass pinned the individual in the trench. The individual was removed from 
the trench by rescue personnel and transported to the local medical center. The scene was secured, and an investigation of the incident commenced. The victim was then ground transported to the local medical center emergency room. Subsequently, it was learned that the patient sustained fractures to both the tibia and fibula of the right leg. Surgery was performed on the evening of March 26. The work in progress in the trenches was to replace canal water lines throughout the complex. Trenches were dug to $\sim 2 \mathrm{~m}$ and backfilled with $\sim 30 \mathrm{~cm}$ of sand to use as a bed for the new piping. Workers had to enter the trenches whenever the trenching operation encountered other buried services. The pinned worker was a foreman who was checking for a potential path around other services. Workers had noticed water accumulations and trickling water on the trench walls, but neither the contractor nor PPPL had the required competent person at the worksite to receive such a report. Part of the trench wall gave way at the foreman's location, and even over such a short distance struck him with enough force to fracture both leg bones. Management did not verify that the contractor was in compliance with OSHA regulations (ORPS CH-PA-PPPL-PPPL-2002-0001).

The DOE formed safety and environmental audit teams, referred to as Tiger Teams, in the early 1990's. These teams of professionals visited DOE sites to observe and audit all types of work activities. The PPPL was visited in late 1990 and early 1991 (DOE, 1991). The Tiger Team found a number of violations of occupational safety and health administration (OSHA) regulations. The break down of the violations were: $46 \%$ electrical, 23\% machine guarding, 7\% hazard communication, 5\% walking and working surfaces, $4 \%$ egress, $3 \%$ hand tools, $3 \%$ personal protective equipment, $3 \%$ welding, and $7 \%$ other issues. One of two important safety findings was that PPPL was not administratively controlling the Kirk keys (safety interlock keys) for engineered safety systems. Late returns of keys were being allowed, which presented a possible electrocution hazard. The other important safety finding was that PPPL did not have the ability to provide a timely and effective response to protect personnel in the event of an emergency requiring electrical isolation. PPPL also acknowledged that they had determined in a self-assessment that their confined space safety program was deficient; they worked to improve the program. In a US fusion experiment, the tokamak interior, the neutral beam lines, the motor-generator equipment accesses, and any equipment wells or underground tunnels are typically confined spaces and must be treated according to US safety regulations.

These occurrences have shown that most of the industrial safety concerns at PPPL are the same concerns shared by many DOE facilities and even those in other industries - the safety issues and concerns discussed in this section are not unique to fusion. Subcontractors do not always follow the DOE or OSHA safety rules although their contracts state that they will comply, temporary workers unfamiliar with the facility can make errors that jeopardize their own safety, and that facility management does not always verify that the subcontract personnel are meeting all aspects of their contractual obligations. Events of working without fall protection and without confined space 
permits, falls from height, severed underground lines, trench wall collapse, small fires, and crane events have occurred. The Tiger Team found that the staff was not always following OSHA safety rules, but PPPL has made efforts to improve their compliance.

\subsection{Other Fusion Machines}

This section presents some of the industrial safety-related events that have occurred at other fusion experiments in the US. These events serve to illustrate several safety-related issues, including the energies utilized in fusion experiments, the operating practices used at fusion facilities, and the nature of non-obvious hazards.

On November 13, 1978, six employees and one visiting scientist working on a direct energy conversion experiment at a magnetic fusion energy research facility in Livermore, California, were exposed to an unmonitored source of x-ray radiation. The direct conversion experiment produced intense x-rays, which had not been predicted by the experimenters or by the Hazards Control Group. The exposures occurred after several changes in the experimental equipment inadvertently eliminated inherent shielding. Three individuals did not observe the radiation source during the experiment, their dosimeters indicated low levels within permissible limits. The dose estimates for the eyes of the other four experimenters were 15, 5.7, 3.1, and 2 rem $(0.15,0.057,0.031$, and $0.02 \mathrm{~Sv}$ ) (DOE, 1980b).

On January 24 and 26, 1980, earthquakes struck near the Lawrence Livermore National Laboratory (LLNL) (Becker, 1982). The first earthquake measured 5.5 on the Richter scale, and the second measured 5.8. The first earthquake had aftershocks of 5.2 and 4.2 on the Richter scale at 53 and $97 \mathrm{~s}$ after the initial earthquake. Laboratory employees responded well, sought cover under furniture or in doorways. They evacuated buildings and trailers without any panic. Of the 65 reported injuries to personnel, 46 injuries were caused by falls or falling objects (note: Becker stated that there were a number of falling objects, including light fixtures, books and materials stored on shelves, etc.). Other injuries were back injuries, lacerations, sprains, contusions, and other minor injuries. The earthquakes did trip electrical circuit breakers and broke fuses, so most portions of the site were without power. The laser facilities appeared to have suffered damage, but it was mostly cosmetic damage that was easily repaired. There was slight optics damage and some structural damage. The laser was back in operation after a month of repairs. The Engineering Test Accelerator was misaligned by the earthquakes. Realignment and other repairs required several weeks at a cost of $\sim \$ 200 \mathrm{k}$.

On June 12, 1980, the Elmo Bumpy Torus fusion experiment at Oak Ridge National Laboratory experienced damage to six of the 24 copper magnet coils. The fusion experiment was located in the Y-12 plant, and was adjacent to a US Nuclear Regulatory Commission experiment. While separate, the two experiments shared the same power supply. The Elmo Bumpy Torus was shut down, with safety interlocks in place to 
preclude operation. Apparently, the electrical safety interlocks failed while the other experiment was in operation; as a result the Elmo Bumpy Torus magnet coils were energized by the shared power supply. The magnet cooling system was not in operation. The coils overheated and six coils were so badly damaged that they required replacement. The Elmo Bumpy Torus was expected to be out of service for two months while the replacement was made. Fortunately, no employees were injured during this unexpected magnet power-up event (DOE, 1981).

On February 13, 1992, a scientist visiting a US fusion experiment at Livermore, California, was rendered unconscious by inhaling nitrogen gas used to cool a diagnostic device. The scientist was trying to align optics on a laser-assisted particle probe spectroscopy diagnostic. As he looked through a hole at the top of a port to the fusion experiment, room light interfered with his work. He placed a black cloth over his head to block out the room light. Nitrogen coolant gas was slowly leaking from the device and it accumulated under the cloth. Other workers noticed that the scientist had passed out and summoned aid. He was transported to the hospital. The scientist was revived from the oxygen deficiency event. An accident investigation was held, and a number of factors were identified. The staff changed from nitrogen to dry air for experiment cooling (ORPS OAK--LLNL-LLNL-1992-0018).

On March 20, 1992, three technicians moved approximately 450 lead bricks to provide shielding from x-ray production on the Experimental Test Accelerator II (ETA-II) at Lawrence Livermore National Laboratory. It was decided to use the opportunity to measure the exposure to lead and/or lead oxide dust during such operations, and to establish data on such operations. The technicians were therefore fitted with air samplers for the entire duration (90 minutes) of the move. The air sampler results were reported as much higher than expected. Following subsequent corrections, the exposures averaged for an eight-hour day for the three technicians were: 104 (plus or minus 12) $\mu \mathrm{g} / \mathrm{m}^{3}, 90$ (plus or minus 11) $\mu \mathrm{g} / \mathrm{m}^{3}$, and 30 (plus or minus 4) $\mu \mathrm{g} / \mathrm{m}^{3}$. Two of these results were above the OSHA permissible exposure limit (PEL) of $50 \mu \mathrm{g} / \mathrm{m}^{3}$ averaged over an eighthour day. However, serious concerns were raised about the quality control on the measurement, both in the laboratory analysis of the samples and in possible contamination of the sample by the technicians involved (e.g., by dust from their gloves). The LLNL Medical Department was consulted. They advised that a one-time exposure at this level is not a hazard to health. The exposed workers were informed that a possible exposure had occurred. They were offered medical advice and tests if desired. A planned movement of 400 additional bricks on April 13, 1992 was used as an opportunity to make more careful measurements. Supervisory personnel were used as brick movers ("Handlers"), in protective gear to ensure that they were not exposed. Each wore two samplers. The person controlling the samplers ("Sampler") wore one, and an Area Sampler was placed $1.5 \mathrm{~m}$ from the lead pile and $1.5 \mathrm{~m}$ from the floor. The personnel were given careful instructions on preventing contamination of the sample. Bricks with visible oxidation were used, although they may not have been as heavily oxidized as the initial set of bricks. A third party reviewed the data and analysis. The results of the more careful measurement were (outside of the respirators worn by the lead brick handlers): 
Handler \#1 - 8 hour averaged exposure was 42 (plus or minus 5) $\mu \mathrm{g} / \mathrm{m}^{3}$

Handler \#2 - 8 hour averaged exposure was 58 (plus or minus 7) $\mu \mathrm{g} / \mathrm{m}^{3}$

Handler \#3 - 8 hour averaged exposure was 50 (plus or minus 6) $\mu \mathrm{g} / \mathrm{m}^{3}$

Sampler - $\quad 8$ hour averaged exposure was 12 (plus or minus 1 ) $\mu \mathrm{g} / \mathrm{m}^{3}$

Area Sample - 8 hour averaged exposure was 17 (plus or minus 2) $\mu \mathrm{g} / \mathrm{m}^{3}$

The quality assurance procedure was completed on April 16, 1992 at 1015 hours. It was concluded that the initial exposures on March 20 were probably in excess of OSHA standards, even though some contamination of the samples cannot be excluded. It was expected that the moving of lead bricks in the amount handled would not expose the workers to lead levels in excess of the OSHA limits. There was no information known to the program to indicate that conditions (e.g., lead oxide dust from brick surfaces) could be such as to permit exposure. These data have indicated a need for a re-evaluation of possible controls on handling lead (ORPS OAK--LLNL-LLNL-1992-0041).

On June 15, 1993, two contractor employees were repairing a cathode tip from the Particle Beam Fusion Accelerator experiment at Sandia when the cathode tip fell about $\sim 2 \mathrm{~m}$ to the floor. The cathode tip missed the two workers and there were no injuries. The cathode tip weighs about $159 \mathrm{~kg}$ and was situated on a lift platform. A working stand was above the cathode tip. One worker began using a chain fall and crane to remove the working stand, the other worker began pushing the lift platform toward the elevator so the cathode tip could be re-installed on the machine. The chain fall's hook caught the cathode tip as it went past and the cathode tip fell to the floor. It was not damaged. The incident was discussed and the procedure was rewritten (ORPS ALO-KOSNL-1000-1993-0008).

On April 10, 1995, a technician received an electrical shock to his left thumb while working at the Particle Beam Fusion Accelerator. He was working on a temporary RF power generator, tracing cables to verify proper system configuration during preoperational testing. As he grasped a cable while physically tracing its route, the cable detached from its connector, leaving the direct current center conductor exposed. The outer braid of the cable was at ground at all times due to the connection at the far end of the cable. The center conductor came into contact with the technician's thumb and he received an electrical shock. The power supply was immediately de-energized. The technician reported to medial, was examined, observed, and released to return to work. Cable examination revealed that the connector had not been adequately crimped, so when the technician grasped the cable, the cable and connector parted. The incident was discussed with the staff and the defective cable was replaced (ORPS ALO-KO-SNL1000-1995-0006).

A summer student was testing the electrical insulation of cables for the field reversed configuration experiment at Los Alamos on June 1, 2004. He was using a 'megger' type crank-operated insulation tester. He placed his left hand on a shelf unit for balance while 
he cranked, and he received an electrical shock. After applying the leads and properly discharging the tester from a previous test, he cranked the tester for about two seconds, heard the clutch slip once and then felt a vibration through his arms and chest. He let go of the handle and the rack immediately. It took him about ten seconds to realize he had been shocked. He felt a slight muscle contraction along the path the current had taken, but there was not any significant reflex action or bodily jerk. He experienced slight cramping in the muscles in the palm of his left hand, where it had been touching the grounded instrument rack. The principal investigator determined that while there could have been $1,000 \mathrm{~V}$ in the wire, there was not enough energy (less than 2 Joules), charge (0.001 Coulomb), current (0.2mA steady, 2A pulsed), time duration (400 microsecond pulse), and effective capacitance to cause a safety concern. The principal investigator, and the task leader, concluded that the cable connected to the Megger had a ground shield that was touching or arcing to the screen room ground, and student was completing the circuit. The event was discussed, and the megger was removed from service to be dismantled so that it could be determined if the unit was malfunctioning (ORPS ALOLA-LANL-PHYSCOMPLX-2004-0001).

There have been a few other events of concern to fusion. Cadwallader (1994) noted that a 1.5-m long high vacuum chamber at the Skeats High Power Lab, operated by General Electric in Philadelphia, suffered a window implosion on September 11, 1986. A technician, who was standing in front of the window, was partially drawn into the vacuum chamber by the inrushing air. He died from asphyxia and chest injuries. This accident with a modest sized chamber caused fusion experiments, with very large vacuum reservoirs, to review and strengthen their approach to vacuum window safety. Plexiglass barriers were placed in front of windows, exclusion areas were re-affirmed for windows that could not accommodate plexiglass, and fusion personnel were briefed on the Philadelphia event.

\subsection{Summary}

The initial assumption at the beginning of this analysis was that fusion experiments would be maintained and operated very well because they are unique and expensive machines, and that any personnel injuries would be atypical events associated with failures of the exotic equipment used in fusion research. The data review has shown that PPPL has had staff injuries and also subcontract workers not wearing fall protection, workers not using confined space permits, occurrences of small fires, subcontract and staff worker fall hazards, spurious halon discharges, staff worker radiation overexposures, underground lines cut during excavation, crane-related events, and even a trench collapse. A valuable insight from all of these event reports is that fusion experiments rely on their "conventional facilities" for all the needed support functions of electric power input, water cooling, gas handling, etc. Fusion experiments operate many systems that are very similar to other industrial concerns, so the safety concerns in other industries are shared by fusion experiments. Aside from the radiation exposure, the 
industrial accidents and events in this chapter are recognized as typical industrial safety problems faced by many industries. Tables 1 and 2 give actuarial data on US industrial safety problems that show falls, being struck by an object, and fires are important, nationwide industrial hazards that create many injuries each year (NSC, 2003). Confined spaces, of which fusion has many, have averaged over 80 fatalities per year, with about one-fourth of those being co-workers attempting rescue (Suruda, 1994). Fortunately, there have not been any fatalities in fusion confined spaces. The other fusion events discussed in this chapter were radiation overexposure, inadvertent magnet power up, oxygen deficiency, chemical overexposure, dropped crane load, and electrical shock. Other than the radiation exposure, these events are also typical of many industrial environments. From the plots given in Figures 1 and 2, the PPPL staff injuries are higher than other DOE operations, but the injuries have not been debilitating. PPPL has been able to operate fusion experiments (the basic machines and their diagnostics), even a successful D-T campaign, safely. It is also positive that PPPL has not experienced any fatalities in the operation of their machines, and that they have made strides to increase the effectiveness of their occupational safety programs after the DOE Tiger Team audit in 1991. PPPL has not had many accident investigation reports filed with the DOE, which indicates that there have been very few accidents of high consequence. The subcontracted workers are suffering injuries of concern around fusion experiments. For a large future machine (such as ITER) that may subcontract hundreds of support employees, proper oversight of the subcontracted workers will be very important for occupational safety. 
Table 1. Occupational Safety Data for US Industrial Workers

\begin{tabular}{|l|c|c|}
\hline \multicolumn{1}{|c|}{ Event or Exposure } & $\begin{array}{c}\text { Private Industry } \\
\text { Count of Nonfatal Cases } \\
\text { for 2001 }\end{array}$ & $\begin{array}{c}\text { All Industries } \\
\text { Count of Fatalities } \\
\text { between 1992-2001 }\end{array}$ \\
\hline $\begin{array}{l}\text { Contact with object or } \\
\text { equipment }\end{array}$ & 400,033 & 9,969 \\
\hline Struck by object & 199,855 & 5,649 \\
\hline Struck against object & 101,177 & 147 \\
\hline $\begin{array}{l}\text { Caught in object, } \\
\text { equipment, } \text { or material }\end{array}$ & 68,048 & 4,137 \\
\hline Fall to lower level & 96,359 & 6,078 \\
\hline Fall on same level & 182,641 & 584 \\
\hline Slips and trips & 50,269 & 16 \\
\hline Overexertion & 409,011 & 67 \\
\hline \begin{tabular}{|l} 
Overexertion in lifting \\
Repetitive motion injuries
\end{tabular} & 227,291 & 40 \\
\hline $\begin{array}{l}\text { Exposed to harmful } \\
\text { substance }\end{array}$ & 65,162 & -- \\
\hline Transportation accidents & 68,269 & 5,623 \\
\hline Fires, explosions & 66,803 & 25,891 \\
\hline Assault or violent act & 3,711 & 1,948 \\
\hline Violence by person & 23,694 & 8,190 \\
\hline $\begin{array}{l}\text { Violence by another } \\
\text { person }\end{array}$ & 17,215 & 2,488 \\
\hline $\begin{array}{l}\text { All other events or } \\
\text { exposures }\end{array}$ & 6,480 & 458 \\
\hline
\end{tabular}

There were 115,933,000 people employed in private industry in the US in calendar year 2001 (NSC, 2003). 
Table 2. Occupational Safety Data for the Electric, Gas, and Sanitary Services in the US

\begin{tabular}{|c|c|c|}
\hline Event or Exposure & $\begin{array}{c}\text { Electric, Gas, and } \\
\text { Sanitary Services } \\
\text { Count of Nonfatal Cases } \\
\text { for } 2001 \\
\end{array}$ & $\begin{array}{c}\text { Electric, Gas, and } \\
\text { Sanitary Services } \\
\text { Count of Fatalities } \\
\text { between 1992-2001 }\end{array}$ \\
\hline $\begin{array}{l}\text { Contact with object or } \\
\text { equipment }\end{array}$ & 2,858 & 153 \\
\hline Struck by object & 1,324 & 81 \\
\hline Struck against object & 910 & - \\
\hline $\begin{array}{l}\text { Caught in object, } \\
\text { equipment, or material }\end{array}$ & 474 & 72 \\
\hline Fall to lower level & 1,122 & 64 \\
\hline Fall on same level & 1,223 & 6 \\
\hline Slips and trips & 678 & -- \\
\hline Overexertion & 3,083 & - \\
\hline Overexertion in lifting & 1,437 & -- \\
\hline Repetitive motion injuries & 470 & -- \\
\hline $\begin{array}{l}\text { Exposed to harmful } \\
\text { substance }\end{array}$ & 775 & 304 \\
\hline Transportation accidents & 1,007 & 463 \\
\hline Fires, explosions & 63 & 82 \\
\hline Assault or violent act & 194 & 50 \\
\hline Violence by person & 54 & 23 \\
\hline $\begin{array}{l}\text { Violence by other } \\
\text { person }\end{array}$ & 140 & 27 \\
\hline $\begin{array}{l}\text { All other events or } \\
\text { exposures }\end{array}$ & 2,398 & 8 \\
\hline
\end{tabular}

Standard Industrial Classification code 49. Employment in this code totaled 852,000 in calendar year 2001 (NSC, 2003). 


\section{Chapter 2 References}

Becker, 1982. R. C. Becker et al., "Impact of the January 1980 Earthquakes on the Lawrence Livermore National Laboratory," Nuclear Safety, 23 (1982) 198-209.

Bol, 1983. K. Bol et al., PBX: The Princeton Beta Experiment, PPPL-2032, Princeton Plasma Physics Laboratory, September 1983.

Bol, 1985. K. Bol, M. Okabayashi, and R. Fonck, "The Poloidal Divertor Experiment (PDX) and the Princeton Beta Experiment (PBX)," Nuclear Fusion, 25 (1985) 1149-1153.

Cadwallader, 1994. L .C. Cadwallader, Vacuum System Operating Experience Review for Fusion Applications, EGG-FSP-11037, Idaho National Engineering Laboratory, March 1994.

DOE, 1980b. Operational Accidents and Radiation Exposures at DOE Facilities Fiscal Year 1979, DOE/EV-0091/2, US Department of Energy, December 1980.

DOE, 1981. Environment, Safety and Health at DOE Facilities, Annual Report Fiscal Year 1980, DOE/EP-0024, US Department of Energy, July 1981.

DOE, 1991. Tiger Team Assessment of the Princeton Plasma Physics Laboratory, DOE/EH-0165, US Department of Energy, March 1991.

Hosea, 1985. J. Hosea, R. Goldston, and P. Colestock, "The Princeton Large Torus," Nuclear Fusion, 25 (1985) 1155-1160.

Lumia, 2002. M. E. Lumia and C. A. Gentile, "Industrial Hygiene Concerns During the Decontamination and Decommissioning of the Tokamak Fusion Test Reactor," Proceedings of the $19^{\text {th }}$ IEEE/NPSS Symposium on Fusion Engineering, January 22-25, 2002, Atlantic City, New Jersey, IEEE (2002) 111-113.

Lumia, 2003. M. E. Lumia, C. Gentile, K. Creek, and R. Sandoval, "Removable Thin Films Used for the Abatement and Mitigation of Beryllium," Proceedings of the $20^{\text {th }}$ IEEE/NPSS Symposium on Fusion Engineering, October 14-17, 2003, San Diego, California, IEEE (2003) 168-170.

Machalek, 1983. M. D. Machalek, "First Plasma Operation of TFTR," Nuclear Technology/Fusion, 4 (1983) 191-193.

Nelson, 2003. B. E. Nelson et al., "Design of the national compact stellarator experiment (NCSX)," Fusion Engineering and Design, 66-68 (2003) 169-174. 
Neumeyer, 2000. C. Neumeyer, National Spherical Torus Experiment (NSTX) Construction, Commissioning, and Initial Operations, PPPL-3414, Princeton Plasma Physics Laboratory, 2000.

NSC, 2003. Injury Facts, 2003 Edition, National Safety Council, Itasca, Illinois, 2003.

Perry, 1999. E. Perry et al., "Decontamination and Decommissioning of the Tokamak Fusion Test Reactor," Proceedings of the $18^{\text {th }}$ IEEE/NPSS Symposium on Fusion Engineering, October 25-29, 1999, Albuquerque, NM, IEEE (1999) 97-100.

Raftopoulos, 2002. S. Raftopoulos et al., "Overview of the TFTR D\&D Program," Proceedings of the $19^{\text {th }}$ IEEE/NPSS Symposium on Fusion Engineering, January 22-25, 2002, Atlantic City, New Jersey, IEEE (2002) 465-468.

Suruda, 1994. A. J. Suruda, T. A. Pettit, G. P. Noonan, and R. M. Ronk, "Deadly rescue: the confined space hazard," Journal of Hazardous Materials, 36 (1994) 45-53.

Tanner, 1982. E. C. Tanner, The First Princeton Tokamaks, An Informal History, 19701980, Princeton Plasma Physics Laboratory, February 1982.

WASH, 1975. Operational Accidents and Radiation Exposure Experience within the United States Atomic Energy Commission, 1943-1975, WASH-1192, US Atomic Energy Commission, Fall 1975. 


\subsection{PARTICLE ACCELERATOR SAFETY}

Particle accelerators have several features and issues in common with magnetic fusion experiments. Both use large amounts of electricity and cryogens. Also, high vacuum is required for both types of machines. Both have radiation concerns, including ionizing radiation and non-ionizing radiation (e.g., RF heating). Shielding is needed to protect against radiation exposure. Constructing a new accelerator that could be several kilometers in circumference is a major construction project, perhaps smaller than, but similar to, the ITER construction activity. Both types of machines operate in campaigns, followed by maintenance outages; however, the accelerators generally tend toward operating for more calendar time per year than fusion experiments. Examining accelerator operating experiences for occupational safety is not only wise from the facility similarities, but also offers potential insights into possible issues for a fusion experiment that operates with higher availability than the present fusion experiments.

\subsection{Stanford Linear Accelerator Center}

This accelerator was planned in the early 1960's and construction began in June 1962 (Neal, 1965). Construction was completed in 1966; the electron accelerator began operating the same year. The accelerator has operated since that time, thus the original accelerator lifetime is approaching 40 years. Other facilities have also been constructed at the Stanford site, including a synchrotron in 1973. Parts of the descriptions of these machines have come from the Stanford Linear Accelerator Center (SLAC) web site. The Positron Electron Project (PEP) was completed in 1980, and is a $9 \mathrm{GeV}$ storage ring; it has been recently rebuilt as PEP-II. It is part of the B Factory, with "B particles" being made from d-quarks and anti-b-quarks. The " $\overline{\mathrm{B}}$ particle" is called B-bar, and it is made from an anti-d-quark and a b-quark. In 1999, a detector was built for the B Factory, it is the $\mathrm{B} / \overline{\mathrm{B}}$ detector, called "BaBar". SLAC employs about 1,200 people (about 150 are doctoral-educated researchers) and annually hosts about 2,500 to 3,000 visiting researchers from around the world. About 900 technical papers are produced annually at SLAC. The annual SLAC budget is about $\$ 170 \mathrm{M}$. In comparison, the PPPL has only about 400 employees. ITER is tentatively expected to be somewhere between these employment levels.

Several sources were surveyed to obtain information on accelerator personnel and operational safety. These reports include the Atomic Energy Commission operational accident reports, DOE reports, and DOE databases on operational events. The accidents and events of industrial safety concern are described here.

One of the initial events cited for SLAC was an apparent radiation overexposure. The accelerator worker's film badge indicated 150 rem (1.5 Sv) over several weeks; however, since the employee had only been performing his routine duties, the film badge was not trusted (WASH, 1975). 
On December 26, 1965, a construction worker was fatally crushed and another was seriously injured with a six-ton concrete plank that fell at the SLAC site (WASH, 1975).

Over the three months of January to March, 1966, an employee received an estimated soft x-ray exposure of $300 \mathrm{rem}(3 \mathrm{~Sv})$ to his foot (at $<35 \mathrm{keV}$ ). There was no evidence of erythema on his foot. The employee had been tuning an RF circulator during that time.

On June 8, 1967, a spectrometer magnet was severely damaged by coil overheating. The magnet had been connected to the wrong power supply. The cost to repair the magnet was $\$ 17 \mathrm{k}$ (WASH, 1975). Fortunately, no one was injured in that event.

On December 7, 1971, two bombs were detonated in the injector sector of the SLAC accelerator, one in the main trigger generator and one in the master oscillator. There was no damage to the main accelerator tunnel and the scheduled startup date for this equipment was not affected. The damage was estimated to be $\$ 45 \mathrm{k}$. The bombs were attributed to student unrest (WASH, 1975).

A fire ignited in the west interaction pit of the Stanford Positron-Electron Asymmetric Ring (SPEAR) facility on March 16, 1975, when a thyratron (a high energy switch that controls high voltage and high amperage) malfunctioned. Four thyratron pulsers, their high voltage power supply, and related cables were either severely damaged or destroyed. Significant damage was sustained by the SPEAR muon chamber in the west pit and also the pit roof. Operation of the ring facility was interrupted for eight days. Operation of the magnetic detector and muon chamber in the west pit was interrupted for approximately four weeks (DOE, 1980).

A construction worker suffered serious burns on July 5, 1978. The worker was steadying a load for a crane operator on the SLAC PEP site. The crane boom came into contact with, or within arcing distance of, a $60 \mathrm{kV}$ power line. The worker's clothing ignited from the flow of current to ground. He suffered $1^{\text {st }}, 2^{\text {nd }}$, and $3^{\text {rd }}$ degree burns over $90 \%$ of his body. His lost workdays while recovering from the burns were recorded as a SLAC occupational injury (DOE, 1980a).

The earliest entry into the Department of Energy Computerized Accident/Incident Reporting System (CAIRS) was March 1977. The initial report was a cryptic description of a chemical explosion that did not injure any employees but did result in $\$ 5 \mathrm{k}$ damage to the facility. The other data in CAIRS is summarized in the lost work day and lost work case plots given in Figure 3, and the injuries are summarized in Figure 4. In Figure 3, lump construction is construction contracted on a lump sum fee basis. The construction work is the same regardless of the type of construction contract. Service contractors can be support services, such as technicians, computer support, custodians, laundry, food 


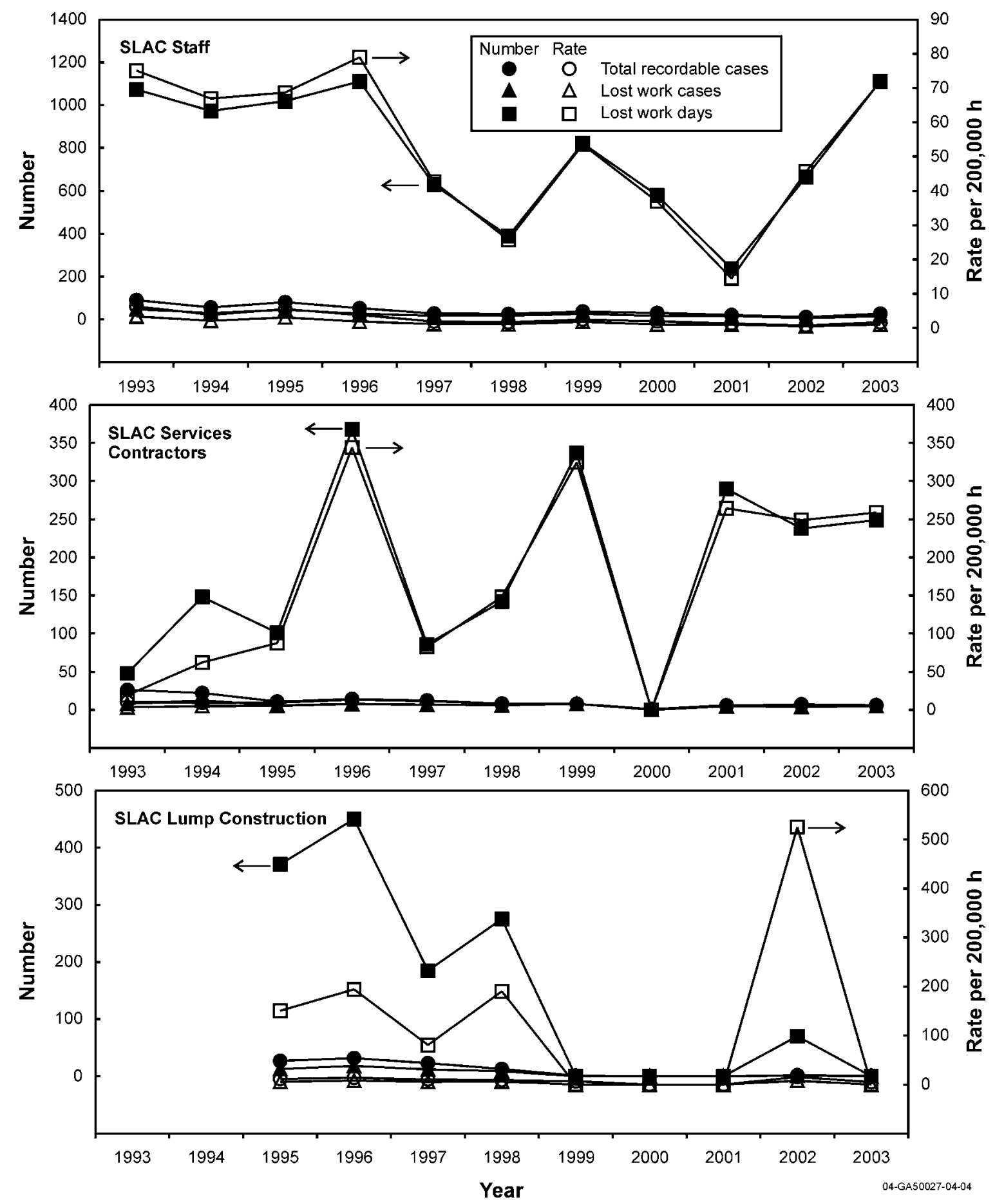

note: There were no fatalities at SLAC in the 1993-2003 time frame.

Figure 3. Plots of SLAC Industrial Safety Performance from 1993-2003. 


\section{SLAC Staff Injuries by Type}
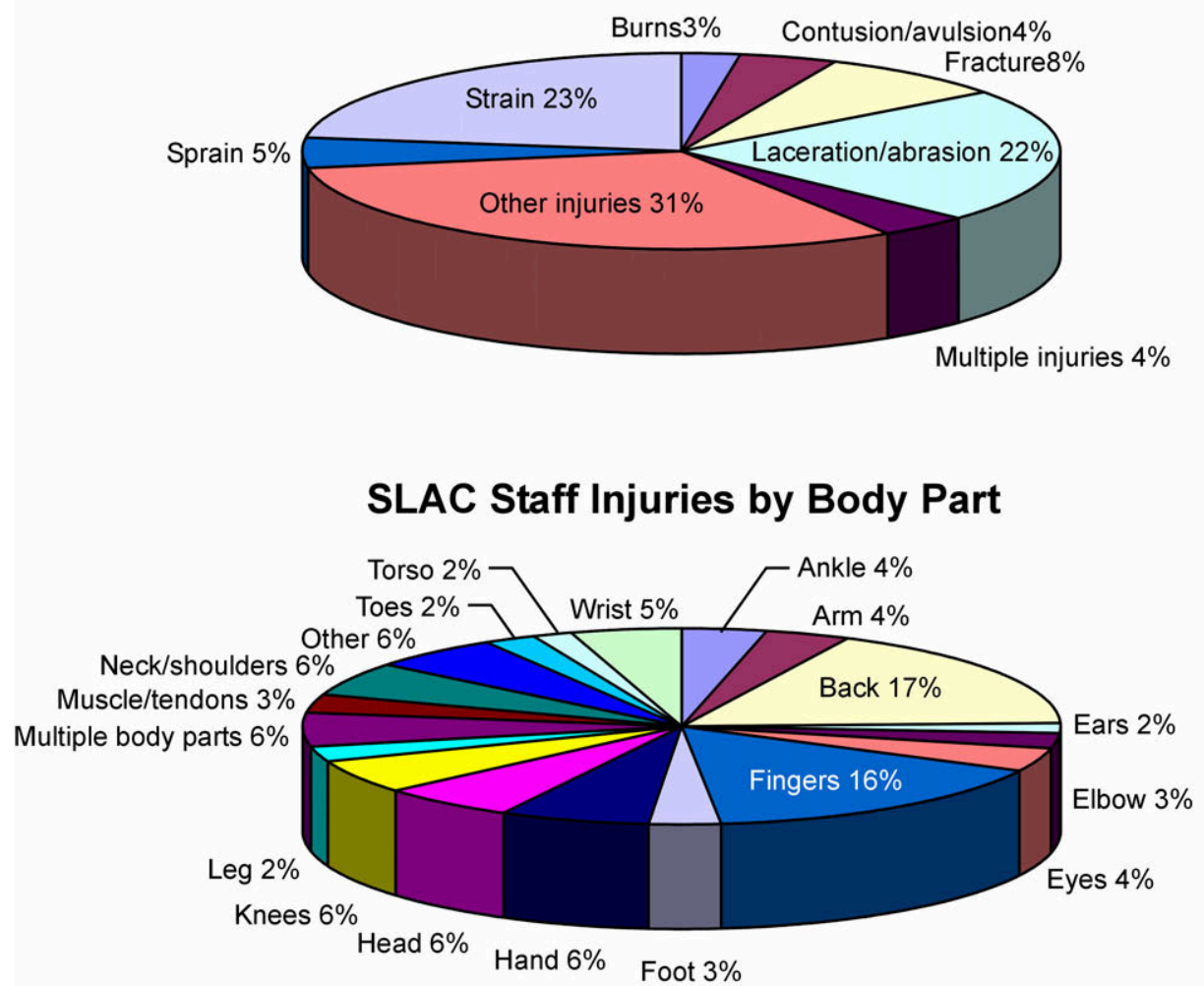

SLAC Staff Injuries by Occupation

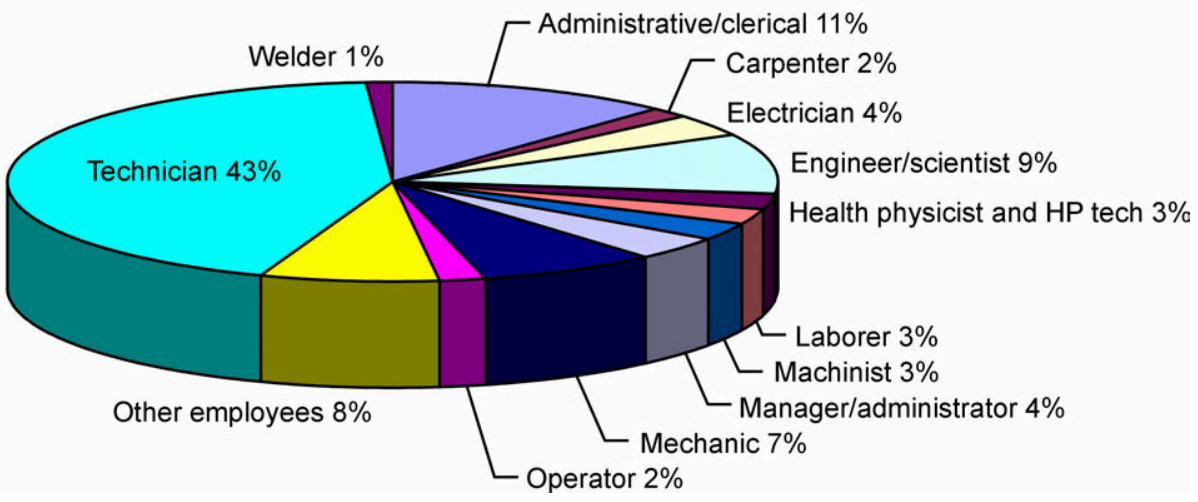

Figure 4. SLAC Industrial Injury Distributions from 1981-2003. 
services, laborers, and other workers. The results show a cyclic trend of decreases and then increases. Reasons for these varying results are not known. Figure 4 shows that the 'other injuries' category is large; it includes issues such as skin irritation, insect bites, puncture wounds to fingers, carpal tunnel syndrome, dermatitis, mental illness, and "injuries not otherwise specified". As seen with PPPL, the SLAC technicians suffer the highest number of injuries, which indicates that again, the technicians are the people who interface most directly with the machine and its subsystems, the work is similar between the two types of facilities. A surprising result is the high number of injuries among the other occupational groups, mainly the administrative staff and the engineers/scientists. The injuries were typically not severe, generally they were small lacerations, falls to the same level (i.e., slipping and tripping), and carpal tunnel syndrome. Nonetheless, the numbers of injuries appeared to be high for the administrative people in traditionally low hazard office occupations. The scientists and engineers probably are injured due to spending time working with the machine and its diagnostics rather than designing, analyzing data, etc. Data similar to that presented in Figures 3 and 4 were prepared for SLAC for the 1985-1990 time period (Lyon, 1991); the earlier data very comparable.

A DOE Tiger Team reviewed SLAC in 1991 as a single-purpose laboratory dedicated to high energy physics (DOE, 1991). In the area of worker safety, a total of 259 noncompliances were found in working conditions related to occupational health and safety. The findings indicated serious deficiencies in the areas of electrical safety standards, hazard communication program, monitoring for regulated chemicals, machine guarding, electrical lockout/tagout, storage of flammable and combustible liquids, emergency egress, confined space entry, and fire protection. The assessment did not uncover any evidence of disciplinary procedures being enforced when workers breached mandatory safety and health regulations. Lyon (1991) reported that the late-1980's SLAC total recordable case rate was about the same as the DOE rate, the lost work case rate was almost double the overall DOE rate at the time, and the lost work day rate was double the overall DOE rate. The reasons for the differences were not clear, but it was obvious that SLAC was having high lost work case and lost work days per accident case.

The DOE ORPS database was searched for events at SLAC. There were 198 off-normal events from 1990-2003. Many were not industrial accidents, they were noncompliances with environmental regulations, missed test intervals, false alarms, procedure noncompliance, equipment failures that did not affect personnel, etc. The events pertinent to industrial safety are described below.

On October 17, 1990, an operator noted that if the rolling radiation shield blocks for section 19 of the positron vault of the accelerator were moved, there was no personnel safety interlock if people were to enter through the opening made by displaced shielding blocks. The issue was reviewed and then the system scheduled to have a personnel safety interlock added in the November 1990 shut down (OAK--SU-SLAC-1990-0006). 
On November 27, 1990, workers were removing an ion chamber assembly from the beam switch yard. An arc occurred when they cut a coaxial cable. The high voltage equipment was not locked out and tagged out per procedure. The employee believed that there were no live conductors in the cable bundle he was cutting, and he had not verified zero energy or lockout-tagout. The worker was not injured but will receive additional training in lockout-tagout (ORPS OAK--SU-SLAC-1990-0011).

On November 27, 1990, during removal of control circuits for rolling concrete blocks in the Final Focus area, wiring technician cut into live $480 \mathrm{~V}$ ac cable and drew an electrical arc. The technician had disconnected and tagged the disconnect switch, but by mistake, he cut into incoming line. The technician should have followed the Lock \& Tag procedure by locking or disconnecting an additional circuit \& checking for voltage before cutting. No one was injured in the arc (ORPS OAK--SU-SLAC-1990-0012).

On December 14, 1990, an employee received an electrical shock to his left hand and arm. He had been testing an energized wire mover assembly in the vacuum building, but he was not following the specific procedures for this type of test. He was exposed to $\sim 900$ Volts. The employee was examined by the SLAC medical office, his electrocardiogram was normal and he suffered no physiological damage from the shock. The employee was counseled about following proper procedures and was given additional electrical safety training (ORPS OAK--SU-SLAC-1990-0014).

On December 27, 1990, an electrician was cleaning components in substation 7 in accelerator sector 14. He received burns to his arms and head, apparently from electrical power in the cabinets he was cleaning. He was taken by ambulance to the Stanford hospital for treatment. The accelerator was down for maintenance at the time of this accident. An investigation committee recommended that the electricians be given better instructions for tasks in their written job orders, wear flame retardant clothing, and improve procedures (ORPS OAK--SU-SLAC-1990-0016).

On January 3, 1991, the plating shop staff smelled nitric acid fumes and they evacuated the plating shop. The shop was externally ventilated and the staff safely re-entered in 2 hours. The shop ventilating system for exhausting fumes from metal finishing operations had suffered fan belt failure. There was no periodic fan belt inspection in place at the time. The fan belts were replaced and a periodic inspection interval was established for the shop (ORPS OAK--SU-SLAC-1991-0017).

During a search of the Damping Ring Intersection Point (DRIP) on February 1, 1991, the operators discovered a problem with the personnel safety system. They determined that it was possible to enter the DRIP via sector 2 or sector 1 doors without safety system actuation (i.e., without dropping the search presets). This lack of actuation would allow unsuspecting personnel to enter the DRIP after searchers had exited, so it was conceivable that personnel could be inside the DRIP tunnel when the searchers secured 
the doors for operations. The access control system will be modified to remove this design flaw OAK--SU-SLAC-1991-0022). It is notable that accelerator safety personnel place a great deal of confidence in the personnel access control systems to maintain personnel safety from machine radiation, yet there are instances where these systems do not protect personnel as required.

On March 4, 1991, a fire occurred in a modulator at test station \#3 in the klystron test laboratory. The plexiglass cover used for personnel electrical protection in the modulator caught fire and the fire destroyed some of the internal components of the modulator power supply. Personnel extinguished the fire. The staff changed the Test Lab to use lockable disconnects on each modulator feed and they have modified the modulator interlocks so that the cabinets cannot be accessed without first locking out the disconnect, so that the flammable plexiglass cover is no longer needed as a safety shield from the internal circuitry (OAK--SU-SLAC-1991-0025).

On May 1, 1991, a North Damping Ring (NDR) distributed ion pump (DIP) high voltage power supply interlock was bypassed. This would have allowed the power supply to be turned on with the NDR in permitted access. The incident revealed a lack of familiarity on the part of some personnel with hazardous equipment interlocks, from both the design and operation point of view. It was also a reminder that non-standard and inadequately documented systems are difficult to troubleshoot and maintain, and that they can contribute to potentially hazardous situations (ORPS OAK--SU-SLAC-1991-1006).

On June 23, 1991, the SSRL Booster to storage ring extraction kicker failed and started a fire. A short circuit in the pulse forming cable caused a resistor to overheat. The root cause was inadequate design safeguards to prevent overheating of the resistor in question. The pulser box directly below the kicker magnet caught on fire. After getting access to the area, Palo Alto Fire Department entered the booster enclosure and extinguished the burning/smoldering components. No one was injured in the fire. Engineering safeguards to prevent fire must be incorporated into the design (ORPS OAK--SU-SLAC-19911014).

On October 4, 1991, employees heard a "bang" emanating from the End Station A (ESA) area. The accelerator was operating and an experiment was in progress in the ESA. Employees entered the area and made a brief visual inspection, but they did not find any reason for the noise. On October 6, other employees entered the ESA and noticed that an aluminum sphere portion of the 3PC3 collimator had ruptured, and pieces were found up to $6 \mathrm{~m}$ away from the unit. The collimator had ruptured due to lack of cooling water. A faulty flow switch had given a false reading that water was flowing but the cooling water was not turned on. The collimator failure resulted in the loss of 3 days of experiment data taking. No one was injured in this explosion (ORPS OAK--SU-SLAC-1991-1026).

On October 10, 1991, four vacuum system metal bellows on a mobile test cart in the klystron lab were found to have induced radioactivity. The contact dose rate was 
$8 \mathrm{mrem} / \mathrm{h}(80 \mu \mathrm{Sv} / \mathrm{h})$ and the dose rate at $30 \mathrm{~cm}$ was $1 \mathrm{mrem} / \mathrm{h}(10 \mu \mathrm{Sv} / \mathrm{h})$. Apparently, radioactive materials were removed from a radioactive materials management area without proper sign-out and labeling procedures. Workers were refamiliarized with proper procedures. The bellows were labeled as activated material and moved to a storage area (ORPS OAK--SU-SLAC-1991-1028).

On October 11, 1991, the hydrogen gas feed system for the furnaces inside the pit furnace developed a leak. The leak was on the hydrogen dryer inside the shack attached to building 025 . The leak vented $1.43 \mathrm{MPa}$ (200 psig) hydrogen gas into the shack. The hydrogen low pressure alarm sounded. No furnace operators were present at the time. An estimated 283 cubic meters (10,000 standard cubic feet) of hydrogen vented. The hydrogen feed was eventually turned off at the tank pad. The hydrogen release was caused by a failure of an elastomeric pressure seal on the body of a particulate filter located in the hydrogen supply circuit of the Pit Furnace. An independent engineering review after the incident showed the cause of the filter failure to be system overpressure, which was caused by regulator failure. The gas did not ignite and no one was injured (ORPS OAK--SU-SLAC-1991-1029).

On October 24, 1991, contract workers were seen in the restricted area of the Beam Dump in the ESA during accelerator operations. Work was stopped. Radiation surveys showed no significant amount of radiation in the area. The radiation padlock keys had been distributed too widely to persons who were not familiar with the access schedule. Workers were allowed to enter while the beam was running. Fortunately there was no radiation exposure to these workers (ORPS OAK--SU-SLAC-1991-1032).

On October 21, 1991, a vacuum technician received an electrical shock when he touched an exposed feedthrough on an operating vacuum ion pump. The technician was examined and was found to be uninjured. The vacuum technician was unfamiliar with the way in which this rebuilt pump was connected. Furthermore, he did not follow a procedure that requires checking high voltage connections for shorts and as a result received a shock (ORPS OAK--SU-SLAC-1991-1033).

On November 1, 1991, a worker was injured while moving material just inside the Final Focus Test Beam (FFTB) tunnel. Two other workmen were in the tunnel at the time but neither saw the accident. The worker was moving a section of $30 \mathrm{~cm}(12 \mathrm{inch})$ channel approximately $1 \mathrm{~m}$ long in the FFTB tunnel toward the sump hole. He hit a small protrusion on the floor and flipped over the piece of channel, landing on his left arm and breaking it. At the time of the report, doctors believed he would require an operation (ORPS OAK--SU-SLAC-1991-1034).

On February 28, 1992, an Industrial Hygienist was called at request of technical supervisor to measure the solvent concentration during an epoxy mixing operation in building 28 of the magnet fabrication area. General room air concentration was measured at $500 \mathrm{ppm}$ of trichloroacetic acid (TCA). The OSHA permissible exposure limit (PEL) is $350 \mathrm{ppm}$, and the OSHA short term exposure limit (STEL) is $450 \mathrm{ppm}$. In a cleaning operation, the TCA concentration in the worker breathing zone measured at 
approximately 1000-1050 ppm, slightly exceeding the $1000 \mathrm{ppm}$ Immediately Dangerous to Life or Health (IDLH) limit. There are no written procedures concerning worker health and safety specifically approved for the Kicker Room in addition to, or integrated with, technical procedures necessary to assemble and/or repair Epoxy Kicker magnets. Management did not react to the change in materials required by the development of epoxy-based kicker magnets as the established production gradually transitioned from room temperature vulcanizing-based instruments. Procedures will be created, and the mixing tank and epoxy tool cleaning operation will be moved to an area with better ventilation (ORPS OAK--SU-SLAC-1992-0009).

On March 10, 1992, a worker was observed standing on the handrail of a platform for the Stanford Large Detector. The worker was not wearing a safety strap even though the distance to the floor was $7.6 \mathrm{~m}$. The worker was accessing an electronics cabinet. The worker safely stepped down from the railing after being given a verbal cease and desist order. The worker stated that he was standing on the railing since it was the most convenient position to access the components being installed in the top of the racks of the cabinets at that location. The worker thought that it was inconvenient to obtain a safety strap belt from the tool crib, so had not done so. Management must be involved to stress the importance of adhering to safety policies and practices (ORPS OAK--SU-SLAC1992-0011). It is notable that while this is clearly a violation of fall protection, the incident also serves to illustrate that designing for maintainability and ease of access to the electronics cabinets will preclude many of these near-miss events. As ITER in-room equipment and systems are laid out, attention to personnel accessibility can promote operational safety.

On April 29, 1992, an RF transmitter in the Klystron Gallery caught fire. The smoke from the fire actuated the smoke detectors. The Fire Department responded and extinguished the fire. No one was injured in this fire. A transformer initiated the fire. The 22-year-old transformer had suffered from degradation of the insulating material, which led to arcing and flames. The firefighters used mono-ammonium phosphate (dry powder) extinguishing agent, which added time to the cleanup process. This compound is caustic to electrical parts and must be cleaned up promptly to avoid damage. (Note: The firefighters may have preferentially used dry powder if insulating oil had been present in the transformer). The total down time was 12 hours of accelerator beam time lost (ORPS OAK--SU-SLAC-1992-0016).

On May 22, 1992, the klystron 6-7 modulator unit sent a fire alarm signal to the control room at 0030 hours. Operators verified that it was a real event rather than a spurious alarm. The Palo Alto Fire Department Engine 7 (the SLAC fire department) was then called to respond. At 0035 hours, the power was cut to the unit, but it continued to burn. The fire department extinguished the fire at 0041 hours, using two portable carbon dioxide fire extinguishers. The fire ignited due to an electrical arc on top of a divider in the modulator cabinet. The arc was probably caused by a combination of dust collecting on top of the divider assembly and an overvoltage condition caused by a voltage regulator failure. The arc set fire to other components in the cabinet, including a $5 \mathrm{kV}$ driver, a 
pulse cable, and other wiring. SLAC will make attempts to clean the cabinets of dust on a regular basis, as operating constraints and personnel reductions allow. No one was injured in this event (ORPS OAK--SU-SLAC-1992-0017).

On October 16, 1992, a SLAC electrician came into contact with a $12 \mathrm{kV}$ line in the Stanford Synchrotron Radiation Lab (SSRL). The electrician involved received burns to his right hand and knee, and a lesser burn to his left knee. He was hospitalized for two days. His co-worker was exposed to an arc flash from the short circuit, but sustained no significant injury. The cause of this accident was the failure of the electrician concerned to follow existing procedures and training in using a high voltage "Hot Stick" voltmeter to determine if there was voltage present before he entered a high voltage cubicle. He read the tags on the switch handle mechanism and apparently assumed that the fuse side of the disconnect was de-energized. He also failed to read two signs on the front of the switch door that warn personnel of the "bottom fed" hookup of this switch. In addition, he had not been instructed to work in sub-station 507 where the event occurred; his assigned work was downstream of the disconnect switch at substation 514. A contributing cause was a defective door interlock on switch A5D, which did not prevent the door from being opened while the switch was in the cubicle. The SSRL and Stanford Positron Electron Asymmetric Ring (SPEAR) were both in a shutdown at the time of this event. Power was lost to the complex for about 2 hours (ORPS OAK--SU-SLAC-19920028).

On January 21, 1993, vacuum technicians were filling a cryogenics bucket with liquid nitrogen (LN2) from a 100 liter dewar in the accelerator tunnel. The valve on the dewar froze in the open position, so the technicians could not stop the nitrogen flow. Shortly after the cryo bucket overflowed, the oxygen deficiency alarm system activated and the tunnel was promptly evacuated. Upon arrival of the Engineering Operator In Charge (EOIC) and the Fire Department, the EOIC requested the Linac tunnel be evacuated from the collider injector development (CID) to S-4. The Fire Department and the EOIC entered the tunnel and cleared the area. No one was found in tunnel. When the 100 liter dewar had emptied, the EOIC checked the area with a portable oxygen monitor and could find no regions less than $20 \%$ oxygen, including near the floor. The EIOC reactivated the oxygen monitoring system and declared the area safe for entry. The direct cause of the event was the liquid nitrogen dewar "freezing" in the open position. The root cause of the valve sticking was water getting into the valve body and freezing when liquid nitrogen flows through the valve. The dewar was stored outside and prior to being used there had been heavy rains that soaked the valve. The lesson learned is that the dewar valves are susceptible to water contamination which can result in the valves "freezing" when liquid nitrogen cools the valve (ORPS OAK--SU-SLAC-1993-0001).

On December 3, 1993, workers were disassembling a polarized gun. A cold trap was placed in a cabinet during the disassembly. The cold trap evolved nitrogen trifluoride as it warmed to room temperature. Nitrogen trifluoride $\left(\mathrm{NF}_{3}\right)$ was not typically condensed in the trap, it was present due to a cryosorption pump containing $\mathrm{NF}_{3}$ being processed. The building was evacuated as a precaution. The root cause was the lack of a procedure for cleaning a cold trap that might be contaminated with material other than the normal 
atmospheric gases encountered in typical operations. The $\mathrm{NF}_{3}$ could pose a health threat, but only 400 cubic centimeters at atmospheric pressure were released. Four workers were examined by the medical department as a precaution, but no injuries were reported (ORPS OAK--SU-SLAC-1993-0015).

On February 19, 1994, the reset button on some water pumps for magnet cooling faulted. The faulted button caused the pumps to trip off line. The magnet continued to operate and overheated, causing the epoxy to melt. No one was injured in this event, but the cost to repair the magnet was \$13k (ORPS OAK--SU-SLAC-1994-0002).

On February 10, 1996, a fire started in a cable junction box. The fire spread along pump power distribution cables, causing about $\$ 200 \mathrm{k}$ in damage to the facility. The direct cause of the fire was breakdown of insulation in a high voltage terminal cabinet, which was caused by the entry of rainwater run off into the cabinet. Heavy rains had saturated the ground and water was coming in to the SLAC buildings. No one was injured in this fire event, but employees were exposed to smoke from the fire, and the accelerator had to be shut down for two weeks to conduct repairs (ORPS OAK--SU-SLAC-1996-0003).

On March 6, 1996, a brazing retort in the Precision Assembly Shop malfunctioned and resulted in an explosion. The retort shell was propelled to the roof and then fell back to the floor. The operator had failed to properly evacuate the retort and backfill it with nitrogen. The hydrogen purge was shorter than the specified 20 minutes. The retort housing and shell were damaged but no employees were seriously injured; one of the two employees closest to the retort had temporary hearing loss and a minor shoulder injury (ORPS OAK--SU-SLAC-1996-0005).

On August 6, 1996, one of two electricians working for an electrical contractor sustained a broken leg and lacerations to the back of the head when a motor control cabinet that they were installing in a klystron alcove overturned. Paramedics took the injured man to the local hospital. The men were attempting to reposition the cabinet on a pallet jack by rotating it 90 degrees so they could move it through an aisle way. As they rotated the cabinet, the pallet jack rocked and tilted; one edge of the cabinet slipped off the fork and it started to overturn. The men tried to steady the cabinet but could not. The electrician's pant leg got caught and trapped under the falling cabinet and he was knocked to the floor. Some damage did occur to the motor control cabinet but the damage had not been assessed at the time of the ORPS report. The event did not disrupt SLAC beam line operations (ORPS OAK--SU-SLAC-1996-0008).

On March 26, 1997, an engineer was testing a prototype modulator design in a high voltage test cage in building 15 at SLAC. Two other workers were present. After turning off the high voltage the engineer neglected to actuate the shorting device used to discharge storage capacitors. Because he had neglected to attach a test probe to the device he rushed to attach the probe. While using two hands to secure the probe he discharged a capacitor through his hand to the other side of his body. He also injured one hand by cutting it on the modulator chassis as he removed it. He was examined by a 
medical staff and was determined to have no injury except to his hand. The root cause of this accident was failure to use proper safety procedures. Safety procedures were not followed due to pressures to complete testing and design in a timely manner. The test program was shut down for three hours as a result of this event (ORPS OAK--SU-SLAC1997-0005).

On April 24, 1997, a painter working for the Plant Engineering Department (PED) Paint Shop received minor injuries when he fell from a scaffold while painting the exterior wall of an electrical substation. The painter had finished painting the east wall and was attempting to step from the scaffold platform on to an adjacent step ladder while still holding his roller extension in one hand. As he placed his foot on the next-to-the-top step of the ladder, the ladder rocked and shifted causing the painter to loose his balance and fall to the pavement below, about $1.5 \mathrm{~m}$. Three electricians working in the next building heard the painter fall, one called the emergency phone number while the others went to the painter's aid. The painter received first aid at the scene from SLAC Fire and Medical personnel and was then taken by paramedics to Stanford Hospital Emergency for treatment. The painter suffered a bump on the head, sore left knee and ribs, and a sore right wrist. He was released from the hospital at around noon the same day. He missed one and a half days of work as a result of the accident. The painter had failed to follow standard practice for the safe use of scaffolds and ladders (ORPS OAK--SU-SLAC-19970007).

On January 27, 1998, a contract worker was pulling a cable into an energized $480 \mathrm{~V}$ Motor Control Center (MCC) unit for the B-factory BaBar detector in the Infrared 2 support building 625 . The wire-way in the MCC, where the cable was being pulled in had been inspected by the foreman on the previous day, but he had failed to notice the energized bus bars and thought it was safe. The worker, a journeyman electrician, was using a metal fish tape to pull the wire. The metal fish tape touched the energized bus at $480 \mathrm{~V}$. That caused an electric arc and tripped the main $480 \mathrm{~V}$ breaker. The worker received an arc flash in his eyes. He was wearing UV safety glasses and leather gloves. He was not injured, and was sent to the SLAC medical unit for examination as a precaution. The B-Factory Safety Officer and the ES\&H Electrical Safety Engineer reviewed the mishap. The work was stopped. The root cause of this occurrence was inattention to details. The foreman checked the job a day before and did the job planning. Then he gave the job briefing to the electrician. But he failed to notice the exposed hot bus underneath the cluster of wires. If he had looked carefully with a flashlight, reviewers believed he would have noticed the hazard. Then adequate precautions could have been taken and the incident would have been precluded. The journeyman electrician also took for granted that there was no hazard, and was not paying attention to the work (ORPS OAK--SU-SLAC-1998-0001).

On April 23, 1998, a subcontract employee reported that he was ascending a fixed metal ladder with a tool belt slung over his left shoulder and a laptop computer handle held between his left thumb and forefinger. While holding onto the ladder with his left hand, he grasped the next rung with his right hand. As he lifted his right foot, the weight of the tools and laptop shifted. He lost his balance and grip on the ladder rung. The individual 
shed the tools and laptop, to no avail. He fell $2 \mathrm{~m}$ (6.5 feet) from the ladder and sustained a fracture to the right femoral bone and a hairline fracture to the L2 disk in his lower back. At 1058 hours on April 23, 1998, a 911 Operator received a call requesting medical assistance at SLAC Building 120 of the SSRL. The subcontractor stopped work. The employee was released from the hospital on April 28, 1998 at 0930 hours. The employee knew climbing a ladder in this manner was not safe and against procedures, but he had climbed with this same equipment many times without accident, so he believed it was a low risk act (ORPS OAK--SU-SLAC-1998-0002). It is notable that this event typifies the issue of worker risk-taking attitudes. The worker in question believed that he was not taking a great risk since he had climbed the ladder improperly many times without any adverse results. Since there seemed to be no adverse consequences to his ladder climbing with tools and laptop computer, he had adopted an unsafe, time-saving approach as his standard approach, and in this instance he experienced the hazard of falling. He dropped the laptop computer and tools so that he could try to stop himself from falling, and we realize how desperate the worker must have been to allow a computer he was responsible for to drop $\sim 2 \mathrm{~m}$. With any new facility startup, there is the opportunity to ingrain proper work attitudes. Perhaps training and enlightenment, or enforcement, or both, are needed, but the new ITER facility should start operation without any worker risk-taking habits.

On May 19, 1999, high radiation was noted around a klystron. During a routine radiation survey in the Klystron Test Lab on May 19, 1999, an Operational Health Physics representative encountered a high radiation reading near the collector of a new 5045 klystron, operating in Test Stand 9. After checking instrumentation, the radiation level was found to be greater than $2 \mathrm{rem} / \mathrm{h}$ on contact. The stream of radiation was approximately $2.3 \mathrm{~m}$ (7.5 feet) off the ground and the direction of the stream was towards the West wall of the Test Lab away from the walkways. The high radiation level was the result of a missing piece of lead shielding (known as the horseshoe) on the klystron where the cooling water exits the collector. The test stand was shut down, the shielding put into place, a new survey showed normal radiation levels and processing continued. The klystron had been installed on May 14, 1999 and processed in voltage per Klystron Test Note number 5 at a 1 microsecond pulse width. On May 18th the testing reached $350 \mathrm{kV}$ at $180 \mathrm{~Hz}$. The test technician performed a radiation survey at this time. His survey showed no unusual radiation dose rates around the klystron. The test technicians' primary dosimeter readings were less than the minimum detectable. The direct cause of the radiation field hazard was a missing horseshoe shaped piece of lead shielding (painted yellow to stand out). The shielding had been overlooked during installation of the klystron. There were no injuries, the two test technicians at test stand \#9 had personal dosimetry, and those dosimeters read no whole body exposure for this time period (ORPS OAK--SU-SLAC-1999-0003).

On September 16, 2000, a smoke alarm sounded in a section of the PEP-II storage ring (PR04) while the colliding beam program for the BaBar detector was operating. The stored beams were dumped and the region was put into controlled access. The firefighters were escorted into the ring tunnel by the chief operator on duty. After a couple of minutes of searching, they found a small fire on some isolated cables that 
service the Longitudinal Feedback Kicker (LFBK) for the Low-Energy Ring (LER). The fire was put out with a hand-held carbon dioxide extinguisher. There was some ancillary damage from the fire to a water hose. The hose was replaced shortly after discovery. There was no other damage to the accelerator or other support systems. The fire was caused when an RF coupler box overheated, igniting the coaxial cable. Installing the very early smoke detection apparatus (VESDA) system proved to be very valuable in alerting personnel to this small fire before more damage could occur. No one was injured in this fire event (OAK--SU-SLAC-2000-0005).

On July 12, 2001, the Area Radiation Monitor in Building 44 actuated, causing an audible alarm. The Test Stand \#2 (TS2) operator helped the person working on test stand 3 (TS3) by surveying a Research \& Development (R\&D) device being tested on TS3. The TS2 operator found excessive X-ray output $(\sim 1.9 \mathrm{rem} / \mathrm{h}[19 \mathrm{mSv} / \mathrm{h}]$ at $30 \mathrm{~cm}$ from the collector) when the tube voltage was at $150 \mathrm{kV}$. The person working on the TS3 was an engineer from a company involved in the design of the R\&D device. He had a visitor badge and had been operating the device by himself without escort. The operator asked the engineer to stop the operation. Meanwhile, the Klystron Radiation Safety Officer (KRSO) was informed about the event. It was noticed that no lead shielding was in place around the collector at TS3. The operators later stacked lead bricks around the collector. During that work, the KRSO informed the responsible physicist for TS3 that the test could not be restarted without first getting the safety documents completed and approved by the KRSO. However, no further administrative control measures were taken and at this point the engineer from the company still was not properly informed of the relevant safety procedures. The next day, Friday July 13, the engineer was again operating on the test stand when the Operational Health Physics Field Operations Group Leader came by around 1500 hours in his walkthrough of the Building 44. The Group Leader found that the engineer was not trained and qualified to operate the TS3. The Group Leader immediately asked him to stop the testing and obtained both his dosimeter and the area dosimeter to check their accumulated doses (fortunately, both were found to be zero). The testing was halted until a full review could be completed. There were both specific violations of procedures, and inadequate procedures for administrative control of the Klystron Test Stand modulators. There was also a lack of communication and enforcement of existing policies (ORPS OAK--SU-SLAC-2001-0003).

On August 28, 2001, an employee of a subcontractor to SLAC was using an electrical grinder with an abrasive wheel to grind on a concrete shielding wall. The grinding operation generated a heavy cloud of dust. A SLAC Industrial Hygienist (IH) conducted a twenty-minute area sample for particulate materials, and crystalline components of the concrete dust. The area sample results indicated that the area near the worker exceeded the Occupational Safety Health Administration (OSHA) Permissible Exposure Limit (PEL) for total silica particulates by a factor of four times with an estimated job length of fifty minutes. Based on the twenty-minute area sample it is likely that the worker may have been overexposed to silica dust. The worker was wearing a disposable dust mask respirator. While the Prework Hazard Analysis did provide for the subcontractor to wear dust masks, there were no details with respect to the sanding of concrete as a step in the 
work process. The root cause of the incident was inadequate work planning for sanding of concrete on the part of the subcontractor. The Pre-Work Hazard Analysis lacked sufficient definition of the work, and the safety procedures defining the personal protective equipment (PPE) were not appropriate to the hazard of the work process. Long-term exposure to silica dust can cause a variety of respiratory diseases including silicosis, pulmonary fibrosis, and emphysema. Short-term exposure to silica dust has not been found to produce significant adverse health effects. If an overexposure occurred (since an area sample was taken and not a personal air monitoring sample the exposure to the employee can't be established precisely), it is believed that it occurred over a relatively short period of time (while the length of exposure can not be established precisely, best information is that it was on the order of hours) (ORPS OAK--SU-SLAC2001-0004).

Between 0840 and 0847 hours on June 18, 2002, a SLAC subcontractor employee sustained a left elbow compound fracture and right knee hematoma after falling off an extension ladder. It is estimated that the individual fell approximately six and one-half feet to the ground. The individual was seen lying on the ground at approximately 0847 hours by a SLAC Technical Division employee. The SLAC employee spoke with the individual and then called an Emergency 911 Operator called at approximately 0851 hours. The individual was transported to Stanford University Medical Center by the Palo Alto Fire Department. The individual was released from the hospital on June 20, 2002. While preparing to apply yellow paint to a Jib crane at Sector 20 of the SLAC Klystron Gallery, the subcontractor employee placed the top rails of a $4.8 \mathrm{~m}$ (16-foot) extension ladder onto the web (mid-section) of the crane's boom. The boom was in its full electric stop position next to the Gallery wall. Even with the boom in this location, sufficient force can be applied to move the boom closer to the wall and beyond the "electrical disconnect closed' position. In this incident, force was applied to the boom causing the boom and mast to rotate towards the Gallery wall. The force was supplied by the individual's ascent. When the individual's upper body approached the top of the ladder, sufficient force was generated to make the boom move closer to the wall. The individual stated that he realized that the rails had slid off the boom. The ladder fell towards the ground, striking the wall. The individual rode the ladder down approximately four feet until he fell off the ladder and struck the ground. The root cause was ruled to be inattention to detail (ORPS OAK--SU-SLAC-2002-0004).

At approximately 0905 hours on August 20, 2002, a SLAC employee sustained a right shoulder dislocation and fracture after tripping and falling forward into a fixed ladder access opening within the Region 4 Positron Electron Project (PEP) utility shaft 4BU. The individual's right shoulder struck a landing, which is located next to the access opening, and his left foot caught the rungs of the ladder. The individual fell approximately four feet and did not fall completely through the opening. The injured employee was observed in the aforementioned position by a coworker who had just turned around after the incident occurred. The coworker helped the injured employee out of the opening and drove him to the SLAC Medical Department, who in turn contacted the Palo Alto Fire Department for transportation to Stanford University Medical Center. The individual was treated and released from the hospital on August 20, 2002. He was 
instructed to return to the hospital on August 22, 2002 to have a metal plate inserted in his shoulder. The individual was required to stay overnight for this procedure and was released from the hospital on August 23, 2002. The course of events before the accident were that after assisting another employee with changing light bulbs within the Region 4 PEP utility shaft, the SLAC employee took a broom and reached overhead to sweep away cobwebs that had accumulated within the shaft. As the employee swept, he also walked around the confines of the landing. The employee stated that he tripped and fell towards the fixed ladder access opening. There were no objects on the floor that contributed to the fall. The fall was on a level surface. The direct cause of this incident was due to an inadequate work environment. As defined by OSHA, a fixed ladder access opening shall be guarded by a standard railing with toe board on all exposed sides (except at the entrance to the opening), with the passage through the railing either provided with a swinging gate or so offset that a person cannot walk directly into the opening. The fixed ladder access opening in PEP utility shaft 4BU was not equipped with a swinging gate nor was it offset. If a gate had been present, the employee would not have fallen to the floor nor entered the fixed ladder access opening. A contributing cause of this incident was inattention to detail. While performing work overhead and looking up, the employee was walking. If the employee had been standing still while performing the overhead task, then it is unlikely that he would have tripped. The root cause of this incident is that the OSHA standard was not brought to the attention of management prior to, or during, the construction of the PEP utility shaft (ORPS OAK--SU-SLAC-2002-0009).

At approximately 1700 hours on September 16, 2002, a SLAC employee and a Stanford University student experienced a near miss incident after a 1.04 by $1.8 \mathrm{~m}$ (41 by 72 inch) by $0.32 \mathrm{~cm}$ (one-eighth inch) thick masonite (a type of wood fiber board, like plywood) window covering fell $6 \mathrm{~m}$ (20 feet) to the floor. The covering landed on the floor and within $30 \mathrm{~cm}$ (one foot) of the SLAC employee. There were no injuries sustained in this incident, which is described as a near miss incident. The masonite was held in place over a window with wood screws that had been screwed through the masonite and into the aluminum window frame. Over time, the screws had loosened and eventually the masonite fell to the ground. The staff resolved to use sheet metal screws on aluminum window frames in the future (ORPS OAK--SU-SLAC-2002-0010).

On July 9, 2003, at 0006 hours, a fire broke out in the Next Linear Collider Test Accelerator (NLCTA) Station 1 klystron high voltage modulator. The fire was caused by an electrical failure inside the pulse forming network cabinet. The interlock system deenergized the unit within a few minutes, but the fire continued to burn. Within 5 minutes, the fire alarm signaled the fire department. They responded to the fire. By 0120 hours, the fire was extinguished. All of the equipment inside the pulse forming network cabinet was destroyed. Some support equipment and cables above the modulator were damaged. During the fire, a nearby water hose was ruptured from the heat and $0.75 \mathrm{~m}^{3}(200$ gallons) of water spilled into the $2.5 \mathrm{~m}^{3}$ (650 gallon) oil tank and the associated secondary containment tank upon which the modulator sits. Approximately $0.37 \mathrm{~m}^{3}(100$ gallons) of displaced oil spilled onto the floor around the modulator. The oil remained confined in the building. The repair of Station 1 was estimated to require about 2 months. During this time, one of the other NLCTA modulators will be used to provide 
Radio Frequency (RF) for accelerator structure development, minimizing the programmatic impact. The cost of the repair is expected to be about $\$ 30 \mathrm{k}$. No one was injured in this event (ORPS OAK--SU-SLAC-2003-0002).

On August 20, 2003, at approximately 1615 hours, a transformer failed in Variable Voltage Substation 11 (VV11), causing a loss of power to 16 klystrons in the linac and an interruption to the scheduled accelerator program. A SLAC security guard reported seeing smoke coming from the Sector 21 Klystron Gallery alcove that houses the transformer. The on-site Palo Alto Fire Department crew and SLAC electricians responded to the scene. After the electricians had confirmed that all power was off to the substation, the transformer cabinet was opened, revealing that the insulation was burning at the top of the coils. The fire crew extinguished the flames. Approximately seven hours later, accelerator operations resumed at a reduced beam energy, using only the remaining VVS's to power the linac. On August 23, a replacement transformer was installed and full power operation was restored. The transformer that failed had been installed specifically to test whether the long-term performance of a dry-type, fixed-ratio transformer would be satisfactory in the SLAC linac application, and whether others of this kind should be procured for use as replacements in other aging linac substations. This incident demonstrated that this dry-type transformer lacked the robustness required for long-term reliability in this application. The klystrons in the SLAC linear accelerator are powered by sixteen variable-voltage substations (VVS's), which were installed more than 25 years ago as part of the original accelerator project. The VVS's are distributed along the Klystron Gallery, one for each sector-pair, plus an additional one for the injector area. Each VVS transforms $12 \mathrm{kV}$ input power to approximately 600 volts, and distributes this power to the klystron modulators. As the name implies, the output voltage is variable and can be remotely controlled through the accelerator control system. In January 2001, the original variable-ratio transformer was removed from VV11 to replace a failed transformer in another sector-pair that was considered more critical to the accelerator program at the time. A refurbished fixed-ratio transformer, which had previously been procured as a candidate for replacing variable transformers, was then installed in VV11. The fixed-ratio transformer was chosen because it would provide a practical test of whether satisfactory accelerator operation could be achieved with a less expensive fixed-ratio transformer without the variable-voltage feature. The fixed-ratio transformer was a dry-type design, in contrast to the original transformers, which are housed in oil-filled tanks. Dry-type, fixed ratio transformers of this kind cost substantially less than the oil-filled variable type, but the long-term reliability of the drytype design had not been demonstrated. The accelerator has been operated at $30 \mathrm{~Hz}$ for most of the 31-month period since the dry-type transformer was installed. At the time of the failure, the accelerator was operating at $120 \mathrm{~Hz}$ and all sixteen klystrons were running at full power. This transformer had been run in this full-load condition for a cumulative total of about eight months before it failed. Although the exact failure mechanism is unknown, it appears likely that a turn-to-turn short appeared while the transformer was running at full power this would have caused intense local heating, leading to ignition of the insulating material between the windings. This experience suggests that a more conservatively rated transformer is needed for reliable $120 \mathrm{~Hz}$ operation in the future. No one was injured in this event, but if the guard had not provided such timely notification, 
the fire would have burned longer and produced more smoke that would have increased the hazard to plant staff and firefighters (ORPS OAK--SU-SLAC-2003-0003).

The DOE accident investigation reports were also surveyed for any severe accident events that might have occurred at SLAC. Two events were found in the recent past. These events are described below.

On January 28, 2003 at approximately 0930 hours, a SLAC systems engineer received head injuries requiring hospitalization after falling from an A-frame, 3.6-m-tall fiberglass stepladder in building 514 of the SSRL. The employee had been searching for nitrogen leaks on the equipment in building 514; leaks were believed to be the cause of excessive nitrogen blanketing gas usage in the klystron transformers (nitrogen is used instead of air, to reduce moisture buildup in the ullage above the transformer insulating oil). The engineer had walked past two electricians as he entered the building. Less than a minute later, the two electricians, who were nearby on unrelated work, heard a 'thud' and found the engineer on the concrete floor between the ladder and the power supplies. The employee was transported and admitted to the hospital. SLAC immediately stopped work at the immediate location of this incident. The stop work did not affect activities related to the accident investigation or operation of a variable voltage transformer. There were no eyewitnesses to the accident and the systems engineer does not remember any events related to the fall. The Board concluded from the results of its limited engineering evaluation that the Systems Engineer could have moved from the ladder while attempting to access the top of the variable voltage transformer (VVT) section of the old Radio Frequency High Voltage Power Supply (RFHVPS) or that the Systems Engineer encountered circumstances that caused him to move off of the ladder while climbing up or down (ORPS OAK--SU-SLAC-2003-0001). The accident investigation report stated that the original design of the RFHVPS could develop a problem that could cause the variable voltage transformers to overheat and violently disassemble, posing a shrapnel hazard to nearby personnel. The RFHVPSs were fenced to maintain a personnel exclusion area, keeping personnel at a safe distance. The cause of the problem was identified and controlled to prevent recurrence. While the problem has been controlled, the exclusion area remained in effect. The systems engineer was working alone and there were no eyewitnesses. The Board concluded that based on the evidence the engineer lost his footing when he stepped from the step ladder to the elevated surface of the RFHVPS. The ladder was not inspected before use, it was not in good condition, and the engineer stepped sideways off the ladder (DOE, 2003).

On October 11, 2004, a subcontractor electrician was injured at SLAC (DOE, 2004). The electrician was installing a 30 ampere circuit breaker in an energized $480 \mathrm{~V}$ electrical circuit breaker panel in the A sector of the klystron gallery. During the installation, an electrical arc flash occurred at 1115 hours. The arc flash ignited the electrician's clothing and the pressure blew him away from the electrical panel. A laborer, who was present to assist the electrician, was standing behind the electrician. The laborer was knocked to the ground by the arc flash pressure burst. A nearby electrician, who was working on an unrelated task, rushed to assist the electrician. He smothered the flames on the man's clothing. When the laborer revived, the rescuer electrician sent him to call for help. The 
Palo Alto fire department emergency medical services personnel arrived at 1120 hours, stabilized the victim, and transported by ambulance him to a burn center hospital [a helicopter was sought first, but the closest available helicopter was too distant; the ambulance offered the shortest travel time]. The injured electrician had third degree burns on his face, chest, and legs, and second degree burns on his arms; approximately $50 \%$ of his body was burned. This accident was analyzed by an investigation board. The normal working procedure would be to mechanically connect the new circuit breaker, then make the electrical connections. The electrician was making the electrical phase connections first; he had screwed down two of the electrical connections and was making the third connection, to phase A, when the accident occurred. Apparently the third screw was not threading and the electrician pushed the screwdriver with extra force. Under the extra force, the phase A bus jumper bar was moved toward the phase B stabilizing clip, compressing the jumper bar's rubber insulation. This reduced distance and compressed insulation allowed a phase-to-phase short circuit and arc flash. The injured electrician was not using insulated, voltage rated tools, nor was he wearing fire retardant clothing, natural fiber (non-melting) underwear, safety glasses, or a switching hood with face shield. These are the protective clothing items, along with voltage-rated gloves, that are specified in the US National Electrical Code for working on energized systems. The accident investigators could not determine why the subcontractor employees were working without proper personal protective equipment, without a pre-work hazard analysis document, without an electrical hot work permit, and without a job hazard analysis and mitigation analysis document. The board concluded that unsafe conditions and operations had become an accepted part of the everyday way of doing business at SLAC. Some divisions and departments at SLAC were not following the DOE Integrated Safety Management approach; operations concerns were placed above safety concerns. SLAC management placed high emphasis on the need to show scientific achievement in their competition with other high energy physics laboratories for funding from the DOE. This upper management emphasis translated to employees as 'just get the job done' (DOE, 2004). The author notes that blemished safety records and flagrant violation of the DOE safety directives are detriments when seeking DOE funding. In general, the DOE community responds to safety issues with replacement of the operating contractor or the senior management of a facility or site.

These ORPS and accident investigation events have demonstrated that there are hazards specific to the type of facility, such as cryogens, and electrical shock and arcs from the many uses of electricity at the accelerator (particularly the klystrons). Other events were a number of fires, mostly electrical in origin, personnel exposed to chemicals, a few explosions, and several events outlining that strict rules regarding radiation safety are not always adhered to at the facility. There were several falls discussed, and a one event of being struck by an object, and nearly being struck by a falling object. The accelerator personnel are noted to not always follow procedures and subcontract personnel do not always follow procedures nor recognize the hazards of the facility. The subcontractors also do not always follow industry good practices, and sometimes do not exhibit 'skill of the craft' knowledge that would prevent accidents. The permanent staff members do not always provide adequate oversight of the subcontract work. 


\subsection{Fermi National Accelerator Laboratory}

Parts of this description of the Fermi National Accelerator Laboratory (FNAL) have come from the history page of the lab's internet site. FNAL is located near Batavia, Illinois, outside of Chicago. Initial construction began in December 1968. The original accelerator was composed of several sections. Protons are first accelerated by a $183-\mathrm{m}$ linear accelerator that sent $200 \mathrm{MeV}$ protons into a Booster ring (287-m circumference), which accelerated the protons to $8 \mathrm{GeV}$. Then the proton batches entered the $6.4-\mathrm{km}$ circumference Main Ring synchrotron, where they were accelerated to $150 \mathrm{GeV}$. These accelerators are situated on a 6,800-acre site and were the original segments of the laboratory, completed in 1971. As a later addition, some of the protons from the Main Ring could be sent to a metal target to produce anti-protons. The anti-protons are stored in a magnetic accumulator. In 1983, the Energy Doubler, later named the Tevatron, was completed (Johnson, 1987). This is another 6.4-km ring, situated in the same tunnel below the Main Ring. The Tevatron was the first accelerator to use superconducting magnets; the original Main Ring used resistive magnets. The Tevatron accepted $150 \mathrm{GeV}$ protons and the anti-protons, counter currently accelerating them to $\sim 1,000 \mathrm{GeV}(1 \mathrm{TeV})$ and allowed the two types of particles to collide in the region of a collider detector. The Main Injector ring is tangent to the Tevatron; it is another 6.4-km ring that was completed in 1998 and became operational in 1999. The Main Injector was a 6-year, $\$ 230 \mathrm{M}$ construction project. The Main Injector accelerates protons into a nickel target to create anti-protons. Above the Main Injector is another ring, the Anti-proton Recycler, which stores anti-protons from the Tevatron for reuse. The Main Injector sends both protons and anti-protons to the Tevatron, where the countercurrent beams are collided. The Main Injector and the Tevatron magnets are liquid helium cooled, so FNAL has a very large helium cryogenic plant on site. That plant is called the Central Helium Liquefier and it is one of the largest cryogenic plants in the US. FNAL employs a total of about 2,100 staff members and has an annual budget on the order of $\$ 300 \mathrm{M}$. It is one of the largest accelerator facilities in the world, and the largest in the US.

FNAL was searched in the DOE CAIRS and ORPS databases. The data in CAIRS is summarized in the lost work day and lost work case plots given in Figure 5 and injury graphs in Figure 6. In Figure 5, lump construction is construction work contracted on a lump sum fee basis, and cost construction is contracted on an actual cost-plus award fee basis. The actual construction work is the same for either type of contract. The 1990's peaks in construction work were likely due to initiation of the Main Injector construction task. The reason for the peak in the 2000's is probably the construction work for additions to the Main Injector. The FNAL permanent staff graph shows an overall downward trend over the time period. Reasons for service contractor peaks in 1998 and 2002 are not known. Service personnel can be technicians, computer support, custodians, laundry, food services, laborers, and other workers. It is possible that the construction work required more on-site support services. The total for all scientific research in the DOE is presented later in this chapter and comparisons show that FNAL has modest or 

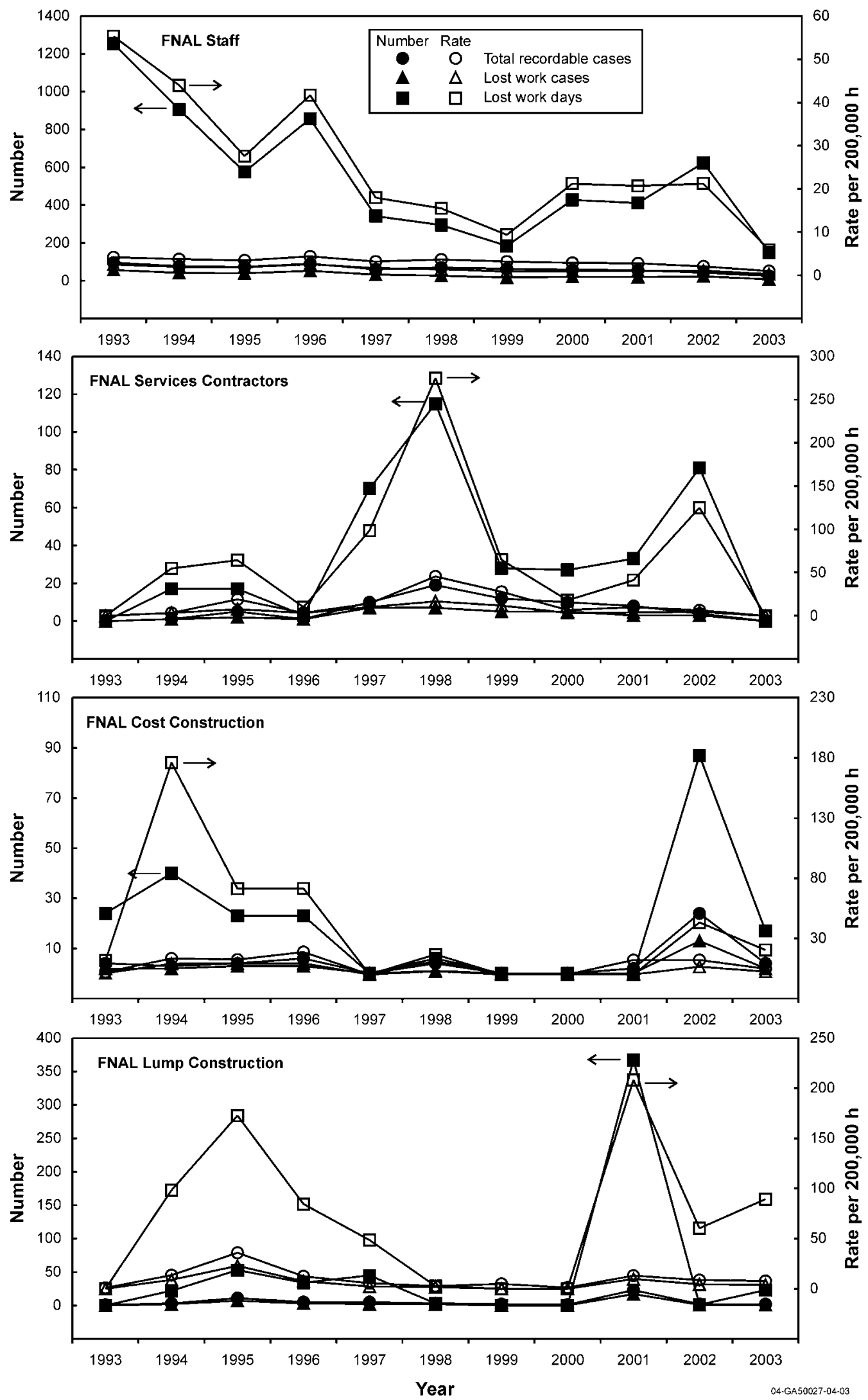

Figure 5. Plots of FNAL Industrial Safety Performance from 1993-2003. 


\section{FNAL Staff Injuries by Type}

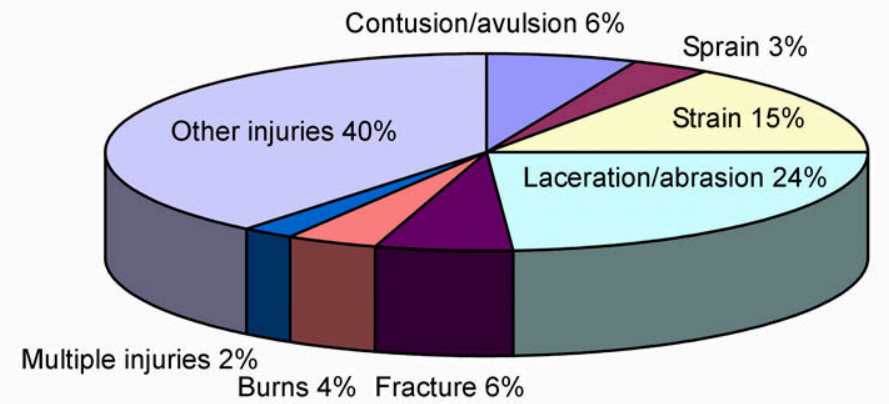

\section{FNAL Staff Injuries by Body Part}

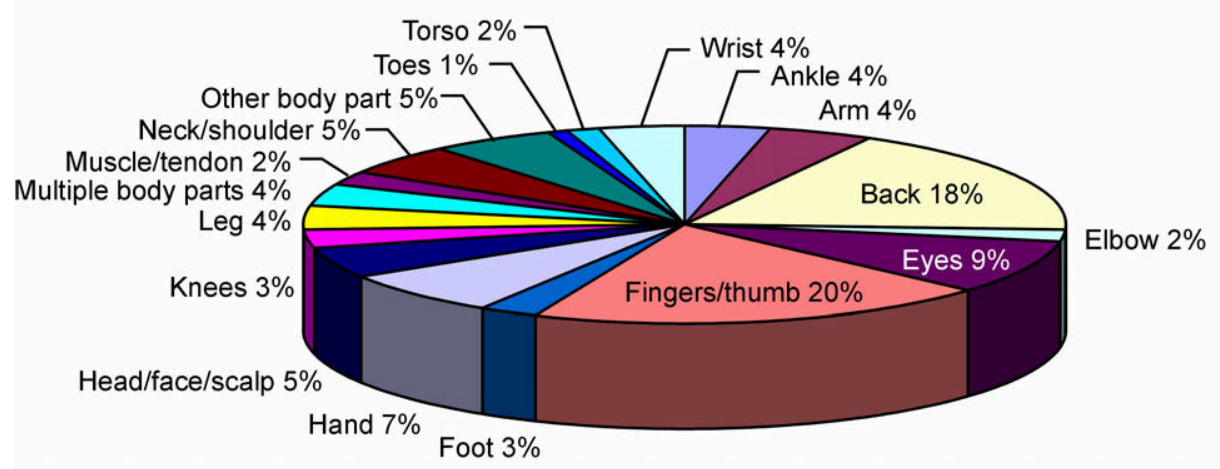

\section{FNAL Staff Injuries by Occupation}

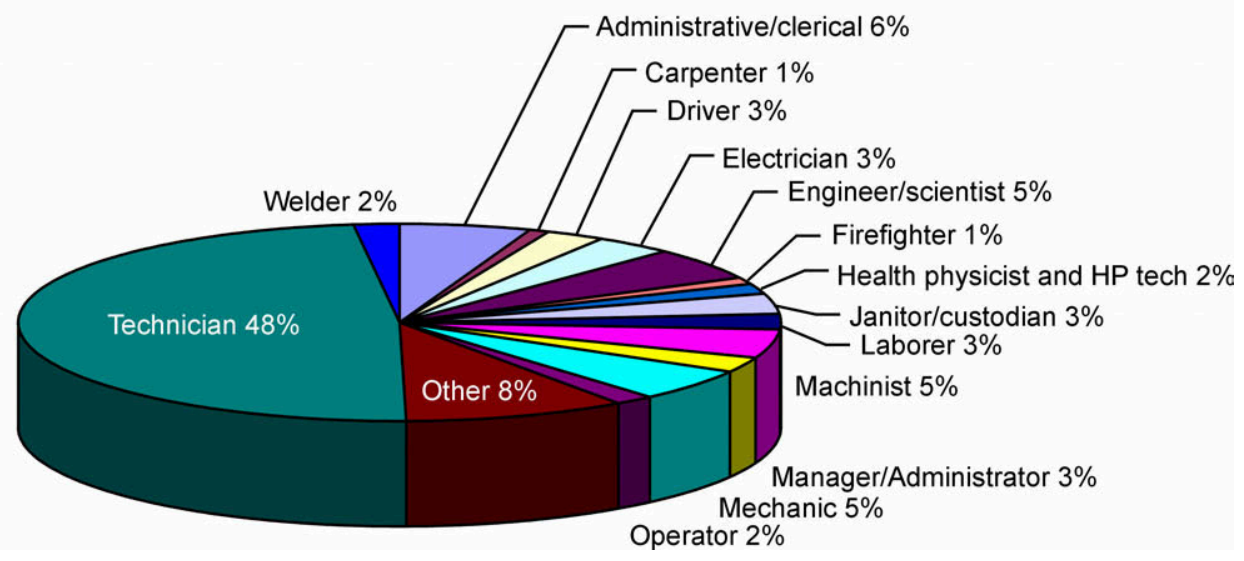

Figure 6. FNAL Industrial Injury Distributions from 1981-2003. 
similar rates compared to the totals for all DOE research and development work. FNAL did not have any work-related fatalities in the 1993-2003 time period, nor were any fatalities noted in the previous DOE operating experience documentation.

Some accelerator operations issues that relate to safety have been discussed in the literature (Mau, 2001; Cossairt, 2000). The Tevatron's superconducting magnets have small cross sectional current flow paths, yet over $4 \mathrm{kA}$ current flows through each magnet. If a magnet transitions to normal resistance (i.e., the magnet quenches due to beam scraping, liquid helium interruption, etc.), the magnet experiencing normal resistance heating from this high current can result in magnet destruction. A magnet takes about one week to replace. The replacement is a time-consuming maintenance task and is costly. Magnet replacement also places workers in the accelerator tunnel, where they are at risk due to cryogen leaks from other magnets that are depowered but not warmed during the replacement outage. Because of the sensitivity of the magnets to quenching, the staff has installed quench protection and beam abort systems. The quench protection system depowers a magnet if the magnet begins to warm up from cryogenic temperature, and the beam abort system quickly sends the accelerator beam to a beam dump if the beam has scraped a wall so that perhaps the magnets near the scrape location will not receive too much heating. These active systems are used to limit magnet damage, which in turn limits personnel exposure to the accelerator tunnel. If personnel must enter the tunnel, the tunnel has been equipped with oxygen deficiency hazard $(\mathrm{ODH})$ alarms in the form of fixed oxygen monitors. These monitors are located throughout the beam enclosures. Each worker who enters the tunnel is required to take a personal oxygen monitor that will alarm if the oxygen concentration drops below $19.5 \%$ by volume. Each worker is also required to carry a small tank that will give them 5 minutes of breathing air, to be used for escape in case of a cryogen leak. Workers must also follow a mandatory FNAL two-man rule for all tunnel accesses. All workers must be certified for tunnel work, with appropriate training and medical approvals to use breathing air tanks. Any heavy work, such as moving magnets, in the tunnel has strict procedures to avoid impacting and rupturing vacuum or cryogenic lines. In addition, the tunnel staff and the Fire Department have been trained in proper response to an ODH emergency. Zinkann (2001) echoed the cryogenic safety concerns, noting that cryogenic lines of helium and nitrogen are routed through occupied work areas and the lines carry large quantities of cryogen. A cryogen release from a pipe break presents both an asphyxiation hazard by displacing air and also an egress hazard. The egress hazard comes from condensation in the room air becoming so dense that people cannot see escape routes through the fog. Zinkann noted that escape lanes have been painted on the floor at his facility to assist in guiding people to exits. There are also room ODH monitors connected to an alarm system; if an $\mathrm{ODH}$ condition is measured then loud claxons and flashing lights will alert personnel to evacuate the area. Cadwallader (2003) has noted that sometimes false alarms occur with oxygen monitors, so voting logic among monitors will preclude most false evacuations. 
Mau (2001) also discussed some other worker safety issues at FNAL. The main tunnel is $6.4-\mathrm{km}$ circumference, it is too long to walk, especially when carrying tools and equipment, or replacement parts, etc. Too much time is consumed for transporting equipment or for an operator to reach an interlock, especially when the experimenters are waiting for beam. Therefore, operators, technicians, and craftsmen use electric golf carts to drive in the ring. FNAL performed a safety analysis regarding cart usage. Fixed bumpers were added at vulnerable areas to prevent golf carts from colliding with cryogenic and vacuum lines. This was a wise precaution because of the potential frequency of occurrence. Cadwallader (1997) has cited some lift truck operator failure rates on the order of 1E-03/demand for a collision during a lift truck task. A golf cart has some advantages over a lift truck, such as the operator has direct line of sight forward, the cart is more maneuverable than a lift truck, and it is likely that the light weight cart can stop more easily than the much heavier lift truck. If lift truck experience can be used, to a first approximation, as an upper bound for golf cart performance, and lift trucks in industrial environments (warehouses, manufacturing plants, etc.) experience monthly collisions, then collision barriers are an absolute necessity in the accelerator tunnel to protect sensitive equipment and cryogenic pipe lines. While golf carts have better visibility than lift trucks, and the tunnel is a single, more predictable path than warehouse aisles or a factory floor, cart drivers can still be distracted by communication devices, pedestrians, parts or equipment placed in the aisle, condensation fog from venting cryogen, time pressure from tight schedules, etc.

Mau (2001) discussed another worker risk issue, the satellite cryogenic stations that cool and pump liquid helium to parts of the Tevatron. These satellite stations are necessary to provide cryogen where needed, but these stations present an operations challenge because they add more rotating equipment in the form of compressors and expansion engines that require constant attention and maintenance. Thus, the maintenance teams spend significant time near the cryogenic equipment for adjustments and repairs. When the workers are in proximity they are at risk of cryogen exposure. Another issue for the operators is that FNAL is very susceptible to power glitches and outages. If the Central Helium Liquefier equipment is not restarted within $\sim 30$ minutes of a site-wide power glitch or outage, then the accelerator is down for a week to purify and recool the ring. Thunderstorms - often accompanied by lightning that can cause power interruptions have a significant operational impact on FNAL. Zankann (2001) also echoed the cryogen production system susceptibility to power glitches. If the cryogenic equipment is not operating to re-condense boiloff vapor, the pressure in the cryogenic system quickly increases and pressure relief valves lift to vent the vapor to protect the system from overpressure. While necessary to protect the system, losing cryogenic gas is costly and impacts the operational availability. There are also personnel safety concerns with venting. If a pressure relief valve fails to operate or develops a freeze plug, the cryogen vapor overpressure could breach the system and possibly vent in occupied areas. If the pressure relief valve operates correctly but the prevailing wind at the time of the release moves the cryogen to building air intakes, the gas could displace oxygen in a building. 
Air intakes should be located away from loading docks, truck and rail receiving areas, the cryoplant, and any emergency power generation equipment. The noise of venting is also significant; workers need ear protection if they must remain near the high noise environment of venting stacks.

Cossairt (2000) discussed some of the 'ordinary' and 'novel' occupational hazards of a new muon facility at Fermilab. The ordinary hazards included high current electrical circuits for the magnets; these require means to effectively lock out power supplies so that the electrical hazard is controlled. RF generation and distribution equipment is extensively used, so engineering and administrative controls to limit exposures would be required. Large numbers of cables in cable trays would be used, so the fire protection concerns are large. Long tunnels present egress concerns, medical response concerns, and fire response concerns. Moving and aligning large, heavy components presents a need to include design considerations that facilitate ease of movement and prevention of injuries. The novel occupational hazards were the large scale use of cryogens in a deep underground facility, requiring a provision to release cryogen to the surface both in normal operations and in the event of magnet quenches. Engineering practices should eliminate the concern for ODH. Another novel hazard is that liquid hydrogen was chosen for the coolant of a neutrino source; the use of a flammable cryogen poses additional hazards than the magnet coolant. Bubble chamber experts were called upon to enhance the safety of the cooling system design. Other novel hazards were the depth and slope of the muon tunnel. The egress points were at the ends of the tunnel; one egress was ordinary and the other was much deeper, so the design called for an emergency egress shaft with an elevator. A 'safe room' was also planned to provide a safe haven in case workers could not reach an exit shaft. The steep slope presents unique hazards. Design consideration was given to making the floor surface sufficiently rough to provide good traction for workers. Gutters were provided to drain seepage water to the sump pits at the lower end. Some type of "speed bump" or other means to retard the downhill movement of rolling items, such as unrestrained equipment, was to be included in the design. Regular tie-down points for heavy items of equipment were provided. Using gutters in a spiral design had been considered, so the gutters drain water and regularly cross the tunnel, serving to direct any rolling items toward the tunnel walls.

FNAL has suffered some large losses and injury events over its 35-year lifetime. Atomic Energy Commission and DOE documents were searched to compile the major occupational injury events. Brief descriptions of these events are given below.

On June 16, 1969, a construction carpenter was fatally injured when he fell $3.5 \mathrm{~m}$ through a floor opening to a concrete floor the next level down. The carpenter fell through an opening that was to be the site of a future stairwell. The temporary cover had been positioned over the opening, but responders noted that it had been removed. No one in the area had seen the cover removed, nor did anyone see the carpenter fall. The carpenter died from basal skull fracture on impact (WASH, 1975). Another carpenter fell from 
height on April 9, 1970. A barn at the laboratory site was being converted into storage space for construction materials and equipment. A ceiling of Styrofoam panels was being constructed on the underside of the joists in the barn. A carpenter fell from the second floor joist level through the Styrofoam ceiling to the barn's concrete floor $2.75 \mathrm{~m}$ below. The carpenter died approximately 11 hours later from brain injuries and hemorrhage resulting from the fall (WASH, 1975).

On January 10, 1973, a subcontractor employee was fatally injured. The employee was attempting to start an earth compactor located in a trench at the FNAL site. A four-wheel drive vehicle was driven to the edge of the excavation trench to provide a jump start for the earth compactor. After the jumper cables were attached, the vehicle rolled forward into the trench and pinned the employee against the earth compactor. The employee died approximately 6 hours later from his injuries (WASH, 1975).

On June 15, 1973, a roof fire occurred. A fuel oil and tar fire occurred during construction of the East Tower of the Central Laboratory and high rise office building. Roofers were preparing to start up an oil-fired roofing tar kettle when vapors from an oil leak in one kettle ignited and the fire spread to the other tar kettles and across the newly installed $195 \mathrm{~m}^{2}$ roof surface. Fortunately, no one was injured. The contractor was responsible for repairs to the roof (WASH, 1975).

On December 26, 1973, a fire began in polyurethane foam insulation in a beam tunnel that was under construction (WASH, 1975). The fire caused $\$ 163 \mathrm{k}$ in damage to the Meson Line Detector building. The cause of the fire was probably a spark or slag from welding operations in a corrugated metal tube that ignited the insulation outside of the tube. The burning insulation produced much smoke and there was concern that the smoke had damaged electrical equipment in the building. Fortunately, there were no personnel injuries.

A severe thunderstorm occurred on September 12-13, 1978 and caused a lengthy power outage. The sump pumps in underground facilities were inoperable due to the power outage and consequent flooding resulted in extensive equipment damage. The storm wind and lightning also caused other damage, with a damage total of \$140k (DOE, 1980). No one was injured.

There were several industrial safety events in 1982. On January 10, there was a period of unusually cold weather, below $-40^{\circ} \mathrm{C}$. A propane heater that was being used to provide supplemental heat stopped working because the fuel froze. The cold then damaged two vacuum pump casings, cracking them. No one was injured. On February 20, the cooling water to a vacuum pump froze because the building's heating, ventilating, and air conditioning unit overheated and then failed. The pump's cooling water froze and the vacuum pump motor burned out. On August 30, 1982, a magnet lift was under way at the 
lab. The crane's choker strap was off center during the lift and a magnet was dropped. There was $\$ 3 \mathrm{k}$ in damage but no one was injured (DOE, 1984).

In 1987, perhaps the most consequential fire in FNAL history occurred. The fire in the Wide Band Laboratory resulted in a property loss and cleanup cost of greater than $\$ 1 \mathrm{M}$, and a programmatic delay of several weeks while amelioration activities were carried out (DOE, 1987). On October 3, 1987, at approximately 0145 hours, a fire was discovered in the Wide Band Laboratory; the accelerator was not operating. The fire involved four particle detectors, the hadron calorimeter, the inner electromagnetic calorimeter, a hodoscope, and a proportional wire chamber. A misaligned ribbon cable connector in the dynode sum box of the inner electromagnetic calorimeter caused an overcurrent in the ribbon cable, which in turn led to overheating and ignition of the cable insulation. The dynode sum box fire spread to several cable runs and to the other three diagnostic detectors. Five automatic fire sprinklers near the ceiling activated; they did not extinguish the fire but did prevent fire spread. The Wide Band lab was being used to study quarks. The FNAL proton beam was to bombard a target to produce a high energy electron beam. The electron beam was to pass through strips of lead in the Wide Band lab and produce a large energy spread of $\mathrm{GeV}$ photons (i.e., a wide band of photon energies) that impinge on a beryllium target to produce quark pairs. After the beryllium target, silicon strip detectors identify and locate the quark states and the quark decay into particles. The wire chambers track electrically charged reaction and decay products. The calorimeters detect photons, pi mesons, and electrons. There is also a uranium calorimeter used on this experiment. The inner electromagnetic calorimeter used $4,090 \mathrm{~kg}$ of lead in an aluminum frame, and $1,136 \mathrm{~kg}$ of poly methyl methacrylate scintillator strips. Thus, the fire spread of toxic materials was a serious concern to the staff and firefighters.

A scientist was examining the gate logic associated with the dynode sum box, which involved going to the experiment hall and disconnecting certain cables. This is the normal procedure for isolating channels of signal. Then the scientist went to the counting room to observe the affected gates. He turned on the voltage and got a signal; this act was per standard operating practices. At about 0120 hours, he returned to the experiment hall (called "the pit"), and he noticed an odor that he described as 'sour milk'. Nothing appeared to be out of the ordinary; he disconnected some ribbon cables and returned to the counting room. In the counting room, he could not get a signal from the gates. He began to examine various systems to identify the problem. During that activity, another scientist left the counting room and entered the pit to inspect wall outlet power, which had tripped earlier in the evening. He saw white smoke and a glow emanating from the pit. He yelled that there was "fire in the pit". A third scientist called the operation center to alert them of the fire, then all four scientists in the area went to the pit to determine the severity of the fire. The smoke density made breathing difficult and flames were visible, so the four evacuated the building. Once outside, two of the scientists secured gas supplies (which included methane gas) to the pit and the other two re-entered the building 
and went to the pit to shut down high voltage power supplies to the detectors. After securing power, they again evacuated the building. Several of the detectors were heavily damaged. All vertical cable insulation was consumed in the fire, and a vertical cable tray had warped and fallen into the debris. Most of the cabling near the detectors was destroyed. The polymethyl methacrylate in the calorimeter melted and burned like flammable liquid; some of the lead in the calorimeter also melted. Aluminum support frameworks melted in the fire; a steel I-beam was also noticeably deformed. The detectors that were held above the floor fell when their aluminum frames lost strength due to the heat. The dense smoke from the fire deposited heavy soot on the building roof and interior surfaces. The sprinkler discharge water on the floor was pumped out; this water contaminated the soil outside the building. No one was injured, although two scientists did re-enter the pit to deenergize electrical power supplies and consequently received additional exposure to the dense, toxic smoke.

The automatic fire sprinklers were about $13 \mathrm{~m}$ above the experiment hall, and from the estimated time of fire ignition about 19 minutes elapsed before the sprinklers actuated. This is to be expected of sprinklers so far above the seat of the fire, enough hot smoke and combustion products must accumulate at the sprinkler head to warm it sufficiently for actuation. However, the sprinklers did perform well despite the distance by limiting the spread of the fire. There were no nearby smoke detectors in that portion of the experiment hall, so the fire had early propagation without any intervention until by chance the situation was noticed by a scientist. The investigators noted that there was no fuse or current limitation on the affected power supply that would have prevented overloading of the ribbon cable. They also noted that the flammability of the cable insulation and the vertical cable runs allowed rapid fire propagation.

In 1992, A Tiger Team visited FNAL, inspecting their compliance with DOE regulations (DOE, 1992). In the worker safety area, there were 161 noncompliances found at the site. The findings indicated serious noncompliances in the areas of electrical standards, hazard communication, respiratory protection, machine guarding, lock and tagout procedures, confined space identification and entry, and storage of flammable and combustible gases. Other concerns included noncompliances with walking/working surfaces, welding, means of egress, and hazardous/toxic substance handling. The Fermilab safety and health program was judged by the Tiger Team to not understand the then-current safety and health requirements as adopted by the DOE. The fact that workers were not recognizing or inspecting for safety and health issues in their workplaces was evidenced in a video often presented to general visitors to the laboratory; the Tiger Team noted several examples of unsafe work practices, all OSHA noncompliances, in that public relations/visitor orientation video. The previous chapter described the Tiger Team findings at PPPL, and the previous section described the Tiger Team findings at SLAC. In the DOE Tiger Team summary (DOE, 1992a), this comparison of numbers of major findings was given: 


\begin{tabular}{|l|c|c|c|}
\hline \multicolumn{1}{|c|}{ Key discipline } & PPPL & SLAC & FNAL \\
\hline ES\&H planning & 0 & 1 & 1 \\
\hline $\begin{array}{l}\text { Formality of } \\
\text { operations }\end{array}$ & 2 & 0 & 1 \\
\hline $\begin{array}{l}\text { ES\&H program } \\
\text { commitment }\end{array}$ & 4 & 0 & 1 \\
\hline
\end{tabular}

When considering these data in view of the operations since the early-1990's Tiger Team visits, it appears that PPPL has strived to address their ES\&H issues, SLAC has suffered several events that has led the DOE to question if they have maintained their alreadyadequate programs, and FNAL has worked to address their ES\&H issues, although they have experienced some electrical injury events.

Several more events of interest came from the DOE CAIRS database. On September 30, 1993, some resistors in the circuit for a transformer overheated when the transformer was being started up, and the resistors caught fire. The fire cost about $\$ 3 \mathrm{k}$ in damage, but fortunately no one was injured in that fire event. On October 15, 1993, a power supply filter overheated and misfired, then ignited. That fire was controlled and no one was injured. On July 7, 1994, a cable in a pulse-forming network sparked and burned its polyethylene covering. No one was injured, but employees may have been exposed to the smoke from the burning polyethylene. On July 26, 1994, a compressor motor overheated and burned out. The fire detection system actuated to alert the staff of the fire; again employees may have been exposed to smoke from this motor fire.

In 1997, two subcontractor electricians at FNAL received serious flash burns as a result of an electrical fault and subsequent electrical arc blast while working on a $480 \mathrm{~V}$ ac motor control center. At approximately 1210 hours on October 22, 1997, the two subcontractor employees were attempting to provide temporary power for lighting and heat from motor control center cabinet \#4 in the RF gallery F-Zero compressor room. The two electricians were removing the upper bus bar cover that shields the line side connections in the electrical panel. The cover was being removed to connect the neutral line associated with the temporary power connection. While attempting to remove the cover, it contacted the " $C$ " phase of the bus bar, causing a short to ground and a subsequent arc blast. The two electricians did not understand that there were energized components behind the cover; they believed that the entire building was de-energized and they did not perform a zero energy check. When the cover movement allowed an arc, one electrician was on a step ladder and his hands were exposed as he held the cover plate, and the other electrician standing on the ground and was exposed to most of the arc, whose energy was directed out and down by the cover plate. The first electrician's hands received second degree burns and the other electrician received second and third degree burns to his face and hands; his hard hat did prevent some facial and forehead injury. When the two electricians recovered their sight after the brilliant flash of the arc, they went outside. Three laborers saw the electricians come out of the building and the 
three laborers proceeded to the nearest building to call for an ambulance. The electricians were transported by ambulance to the local hospital. The electrician with burned hands was treated and released on October 22, and the more seriously burned electrician was also treated and was released on October 27. The investigation board had several findings, including that Fermilab did not have a comprehensive electrical safety program, there was no work documentation and no engineering drawings of the system. Fermilab did not ensure that an adequate integrated safety management system was in place for electrical work. The investigation board found that Fermilab managers did not understand their responsibilities for electrical safety (DOE, 1997).

An unusual type of industrial accident occurred at FNAL on September 4, 1998. The laboratory had contracted to have the concrete floors of several kitchen storage rooms in Wilson Hall repainted. The contractor began the work using a non-flammable cleaner to clean the floors and remove any grease or other foreign materials that would interfere with the painting. The foreman for the job was instructed by his management to use muriatic acid, if needed, to clean the floor. He was later instructed that if the acid was insufficient then to use methyl ethyl ketone. The foreman ordered 'muriatic acetone' instead; the FNAL chemical supply room staff gave him acetone since they did not know what 'muriatic acetone' was. When the foreman and two painters were applying the acetone and water to the floor, vapors were collecting in this unventilated room. Just as the foreman had left the room to empty a mop pail, the acetone vapors must have reached the lower flammable limit $(2.5 \%)$ and were ignited by a floor buffing machine one of the painters had actuated. One painter received first and second degree burns over $20 \%$ of his body, and the other painter received second and third degree burns over $33 \%$ of his body. The foreman received minor burns. The first painter and the foreman were transported by ambulance to a hospital, and the second painter was flown by helicopter to a burn center. After skin graft surgery, the painter was released from the burn center on September 19, 1998. The investigation board found that Fermilab did not perform a job safety analysis for the contract services, and while Fermilab personnel did visit the work site on the day of the job, they did not closely check what chemicals were in use (DOE, 1998).

As part of the Neutrinos at the Main Injector project at Fermilab, new accelerator tunnels were being excavated. On June 21, 2001, a construction subcontractor employee received a serious head injury when he was struck by part of a drilling rig (DOE, 2001). The drill was being used to make six exhaust air ventilation shafts and survey risers for this project. The driller and his helper were bringing drill pipe up to remove the lower portions of the drill assembly. They were opening the jointed between drill pipe sections by using a "tong" (a 0.8-m long steel bar used to torque drill pipe). The tong is connected to a hydraulic system via a wire rope sling, eyebolt and the mechanical connection to the hydraulic piston cylinder. The hydraulic system applies force to the drill pipe sections. A field weld had been made in 1999 to repair the eyebolt at the end of the hydraulic piston cylinder. The repair weld failed during operations on June 21 and the eyebolt 
parted, allowing a release of the torque energy that had been imparted onto the drill pipe. The tong was attached to the drill pipe joint and it rotated with the pipe. The tong struck the right side of the driller's head, just below his industrial helmet, as he was standing near the drill pipe to disconnect the pipe joint. The drilling company did not have records of their repair weld; in fact, the injured operator was the person who had made the repair weld in 1999. Fermilab personnel did not verify that the drilling company was operating up to OSHA requirements, and they did not inspect the drilling equipment nor observe the work for any significant amount of time.

FNAL is the largest particle accelerator in the US and is among the largest accelerators in the world. FNAL has not had any staff or subcontractor fatalities in operations, but the site has experienced construction fatalities. The staff injury values are the same or slightly higher than the DOE averages for research and development facilities, but many of the injuries are not severe (contusions, lacerations, etc.). FNAL has experienced a number of electrical fires, most resulting in only small damage and no injuries. The accident investigation reports have shown that FNAL has had a number of severe occupational accidents, and the investigation boards have pointed out both the good practices FNAL has and its deficiencies in procedures, supervision, and oversight of contractors.

\subsection{Jefferson Laboratory}

A consortium of schools, called the Southeastern Universities Research Association, manages and operates the Continuous Electron Beam Accelerator Facility (CEBAF). CEBAF was renamed the Thomas Jefferson National Accelerator Facility in 1996. The facility is now generally referred to as Jefferson Laboratory or "Jlab". The facility is located outside Newport News, Virginia. The site was selected in 1984 and construction began in 1987. First electrons were generated in this superconducting linac in 1994. The full design energy ( $4 \mathrm{GeV}$ ) electrons were delivered to the experiment halls in 1997. A free electron laser was built and had first light in 1998; in 2000 a $6 \mathrm{GeV}$ electron beam was delivered to the experiment halls. In 2001 an upgrade project began to increase the free electron laser to $10 \mathrm{~kW}$, the upgrade was completed in early 2004. Also in 2004 the DOE approved an increase in Jlab electron energy to $12 \mathrm{GeV}$. The upgrade is under way as of this writing. Jlab employs about 550 people directly and subcontracts some of their support and construction work. The Jlab annual operating budget is on the order of \$70M.

The Jlab occupational safety data are presented in Figures 7 and 8. These data are similar to the other facilities already presented. The Jlab plot annual values are below the DOE research and development values. The staff peak in 1994 was probably due to experiment commissioning tests, and free electron laser upgrade in 2001-2002 may be responsible for the peaks in those years. The pie charts in Figure 8 show that once again, technicians are the leading group for industrial injuries. 

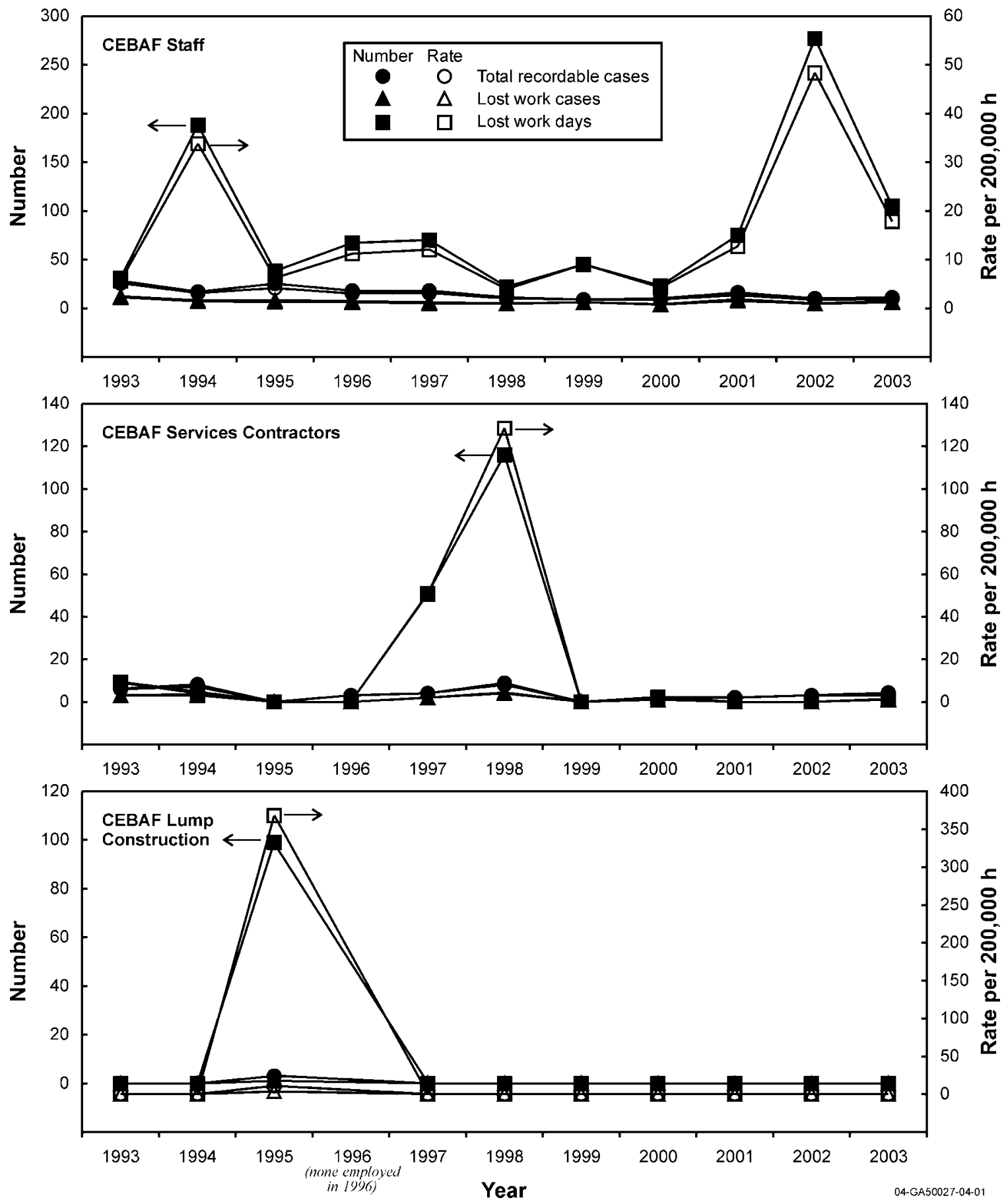

Figure 7. Plots of CEBAF Industrial Safety Performance from 1993-2003. 


\section{CEBAF Staff Injuries by Type}

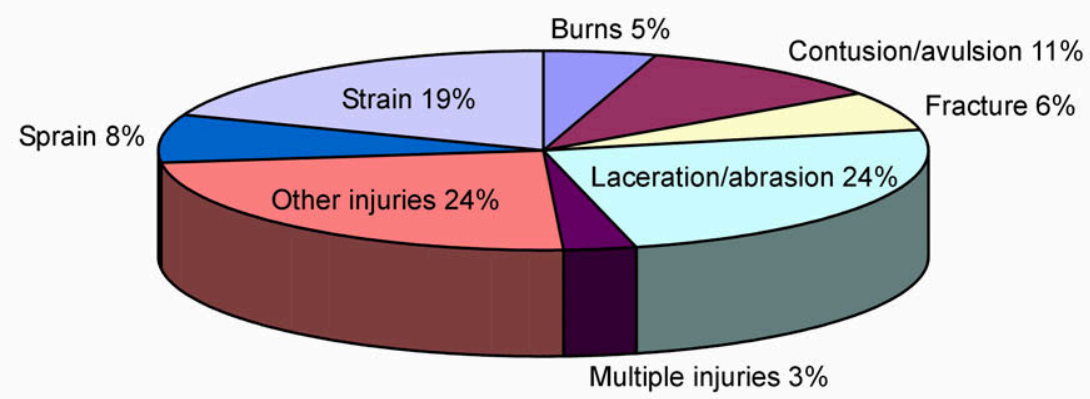

\section{CEBAF Staff Injuries by Body Part}

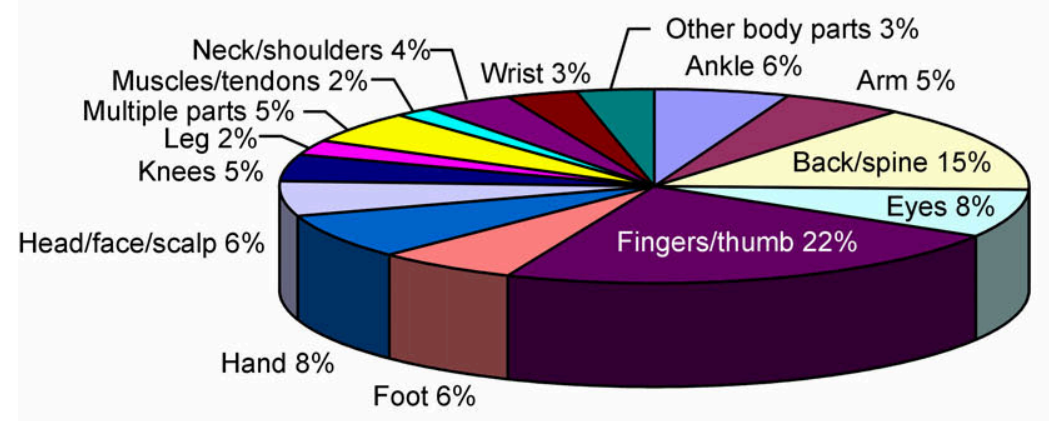

\section{CEBAF Staff Injuries by Occupation}

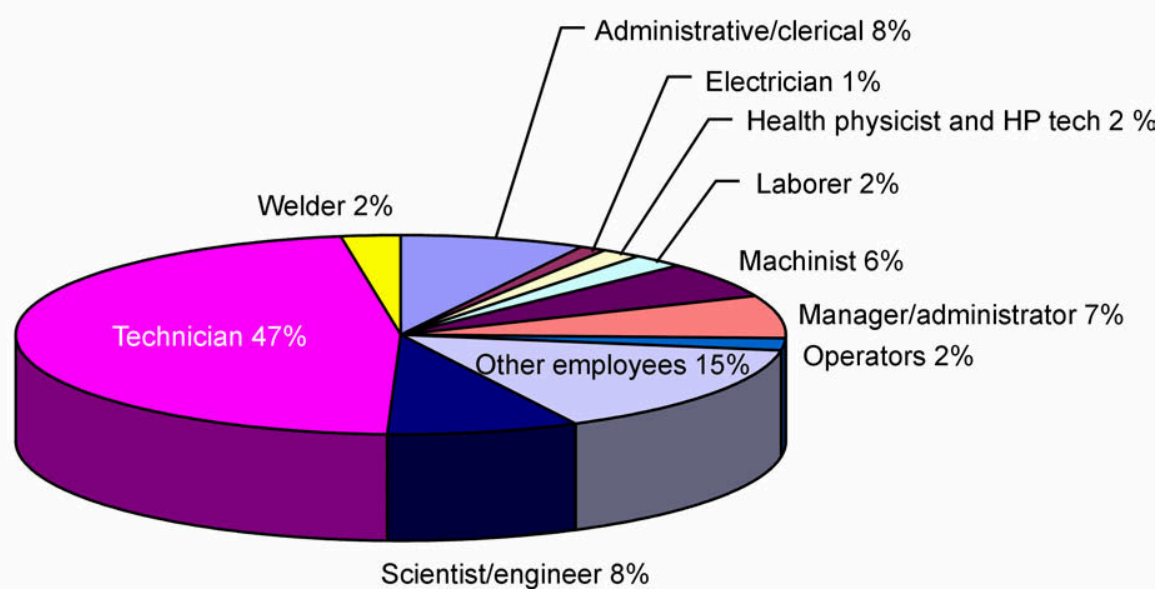

Figure 8. CEBAF Industrial Injury Distributions from 1988-2003. 
There have been several events of importance at Jlab. The DOE ORPS had 41 reports filed by Jlab between 1991 and 2003. The ORPS reports of occupational safety concern are summarized here.

On October 16, 1991, a helium gas recovery compressor in the central helium liquefier system began leaking compressor oil. The estimated $\sim 378 \mathrm{~cm}^{3}$ ( $\sim 0.1$ gallon) of oil impinged on hot parts of the compressor rig and began smoking, but there was no fire. No one was injured in this event (ORPS report ORO--SURA-TJNAF-1991-1002). The compressor fault caused the electrical substation to trip off line and the set of compressors shut down; the compressors were restarted and the damaged compressor was repaired in the next scheduled maintenance outage.

On November 11, 1992, a four-man team of electronic technicians was connecting vacuum rack power supplies and ion pump power supplies to newly-installed cryomodules in the accelerator tunnel. The technicians were working in two-man teams. The technicians were to test the power supply cables prior to beginning physical installation. A subcontract technician was utilizing a high voltage probe and multimeter to check cables. Assuming that the high voltage power supply was off, the technician inserted a male connector into the cable female connector to conduct the tests. The power supply was in fact on; the technician received a shock. He was transported to the local hospital but he had no physiological symptoms of electrical shock, so the technician was released. The technician did not follow the established procedures, but the root cause was found to be inadequate definition and distribution of procedures and policies (ORPS ORO--SURA-TJNAF-1992-0004).

On January 29, 1993, a cable termination in a transformer failed and arced. This fault tripped a 15,000 Volt electrical system and caused $\$ 3.7 \mathrm{k}$ in damage, fortunately no one was injured (ORPS ORO--SURA-TJNAF-1993-0001).

On September 8, 1993, personnel were restarting a large (4160 V, 2,250 horsepower) compressor in the Jlab central helium liquefier. A ball bearing in the compressor suffered a material failure during this startup; the ball bearing seized. The compressor motor began to smoke and the compressor was secured from operations. No one was injured, but employees were exposed to the motor smoke. Investigation revealed the bearing fault and the compressor was replaced, which required about a week of downtime. The helium system ran at $2 / 3$ capacity until the new unit was brought into service (ORPS ORO-SURA-TJNAF-1993-0002).

Accelerator Division Operations Department staff noted at approximately 1030 hours on December 21, 1993 that an RF system interlock had been previously defeated as a portion of a test. This unlogged, defeated interlock allowed RF power to be provided to the North Linac cryomodule, with personnel in the Accelerator Tunnel. Sufficient RF levels to the cryomodule have the potential for producing significant ionizing radiation fields (i.e., field emission x-rays). Two staff members had entered the accelerator tunnel for a 
limited duration activity and had been near the cryomodule. They had used a radiation survey instrument in accordance with existing procedures and had not noted any radiation levels of significance. When the defeated interlock was discovered, all systems were shut down. The staff verified that no personnel were in the area close to the RF source when RF power levels were high. The two staff member's dosimeter badges showed that they had not received any significant radiation exposure from that event. After a thorough review, accelerator commissioning activities were resumed (ORPS ORO--SURATJNAF-1993-0003).

On May 24, 1994, at approximately 1330 hours, an Accelerator Division technician received an electrical shock while working in the CEBAF Test Lab Building (Bldg. 58). The technician was working in the vacuum cleaning area of the Test Lab mezzanine. The technician was attempting to disconnect a portable transducer from an ultrasonic power cabinet. The technician received an electrical shock while she was holding a disconnected connector on the cable leading to the fifth transducer (of the ultrasonic cleaning station). At this same time, the technician was touching the high voltage connector on the back of the No. 3 high frequency generator in the ultrasonic power cabinet. The technician reported the event to a nearby coworker in an adjacent building and she complained of pain in her right arm and shoulder. She briefly described to a coworker her activities leading up to the electrical shock prior to being transported to the CEBAF Medical Clinic for evaluation. After evaluation at the CEBAF Medical Clinic, she was transported to a local hospital for further observation. The technician was subsequently admitted as an inpatient at the local hospital. No significant physiological effects were identified. The initial investigation failed to reveal any readily apparent electrical shock hazard. A Type B accident investigation was conducted, and personnel from the Langley safety office of the National Aeronautics and Space Administration were asked to investigate the equipment. Only three possible explanations were thought plausible; stored electrical charge in the transducer, failure of the ultrasonic unit's electrical contactor to switch off power when the main power switch was turned off, and possible inadvertent contact with an energized 277 Volt or 110 Volt wire in the cabinet. The technician returned to work, and electrical safety training was emphasized for all personnel (ORPS ORO--SURA-TJNAF-1994-0001).

On March 28, 1995, at approximately 1115 hours in CEBAF Experimental Hall A, a CEBAF Physics Division subcontractor employee was struck by a falling tubular steel handrail section. The $26.8 \mathrm{~kg}$ (59 pound) handrail section had fallen approximately $14.3 \mathrm{~m}$ (47 feet) from a stator crane service platform. The employee (a millwright) was conducting calibration testing of alignment equipment with two other coworkers. A 500 ton stator crane had been erected overhead to assist in experimental equipment assembly. At the time of the accident, this crane had been previously tagged and locked out of service. Also in the overhead area of Experimental Hall A was a 20 ton polar crane, this crane was being positioned for an upcoming lift at the time of the accident. The polar crane positioning movement dislodged the handrail section from the 500 ton stator crane. The dislodged handrail section fell and struck the millwright on the right side of his head, shoulder, and back. The impact of the falling handrail section knocked the millwright to the floor, where he remained conscious. A CEBAF staff member initiated the 911 call 
and the CEBAF emergency response plan. The millwright was transported by a City of Newport News medical services ambulance to a local hospital for evaluation. At approximately $2: 15 \mathrm{pm}$, he was transported to another local hospital, Riverside Hospital in Newport News, for further evaluation. At approximately 1800 hours, he was transported to Norfolk General Hospital in Norfolk, Virginia. There he was operated on for scapular and back injuries. The operation was successful and the millwright went to intensive care as a stable patient. He was moved out of the intensive care unit on April 16, 1995 and was discharged from the hospital on April 22, 1995. He then began undergoing physical therapy. Investigation revealed that probable direct cause was that the operator of the radial crane maneuvered the 20 ton trolley of the radial crane so that it struck the handrails of the stator crane 500 ton trolley. Procedures were augmented to account for crane interference in all aspects of Jlab operations. About a week of time was lost to the experiment equipment installation schedule (ORPS ORO--SURA-TJNAF1995-0002).

At approximately 1345 hours on Monday, April 3, 1995, a CEBAF Accelerator Division staff member experienced a close call (near miss) electrical incident. The CEBAF staff member had been tasked to clean-out a utility trench in the floor of the CEBAF Test Lab (Building 58) high bay area. The CEBAF staff member was attempting to remove a section of what he believed was inactive water pipe. This apparent pipe was actually metal conduit containing an electrical conductor (480 volt, 3 phase AC) for a welding receptacle. The CEBAF staff member attempted to cut the conduit with a pair of bolt cutters. An electrical arc flash resulted and the circuit breaker on the high bay area wall tripped. The CEBAF staff member immediately contacted a CEBAF Accelerator Division EH\&S specialist and his supervisor. The worker was taken to the CEBAF Clinic and examined by the CEBAF Physician. The examination did not note any signs of electrical shock or any other type of injury. The worker was released for normal duties (ORPS ORO--SURA-TJNAF-1995-0003).

Jefferson Lab Personnel Safety System (PSS) Group personnel were beginning routine testing of the Oxygen Hazard Deficiency (ODH) monitoring system at approximately 1700 hours on January 15, 1997. The accelerator at Jefferson Lab is a superconducting $\mathrm{RF}$ accelerator and uses cryogenic helium in several areas. These areas are equipped with ODH monitoring sensors to note decreased oxygen levels in the event of helium release. The PSS personnel noted that the alarm did not activate during the test. The circuit breaker that supplies power to the monitors was found tripped. Investigation revealed that electrical maintenance work from late 1996 caused the ODH system to switch to temporary power, and this power transient caused the circuit breaker to open. A power alarm was installed to alert operators if power to the $\mathrm{ODH}$ monitors is lost (ORPS ORO--SURA-TJNAF-1997-0001).

At approximately 0820 hours on October 7, 1998, Accelerator Division Survey and Alignment Group staff members reported that a survey tripod had struck the Hall $\mathrm{C}$ experimental target vacuum window, rupturing the window. Hall $\mathrm{C}$ was evacuated until an evaluation was made by the Hall $\mathrm{C}$ Machine Control Center and Radiation Control Group staff. No personnel injuries resulted from this event, however the potential for 
personnel injury did exist. Radiological surveys showed that no contamination was released when the chamber was breached. The direct cause of this event was determined to be the steel survey tripod being inadvertently drawn into the uncovered target window by an inappropriately energized air core magnet (ORPS ORO--SURA-TJNAF-19980004). Note that this event is not unique to accelerators. Fusion experiments and magnetic resonance imaging magnets have had magnetic field induced missiles as well (Cadwallader, 1991).

The Jefferson Lab Radiation Control Group was notified at approximately 1600 hours on Friday, August 17, 2001, that a radiation monitor in the Vertical Test Area (VTA) was in alarm. The VTA had been processing a component with one of the eight dewars units in operation. The VTA has eight dewars that provide liquid helium cooling of superconducting RF and other cryogenic components used in the production of accelerating structures. RF energy is created testing accelerator superconducting RF (SRF) components. A byproduct of this RF energy is field emission X-ray generation. Operators were testing components at higher and higher power levels, and noted the area monitor alarmed. When it would not reset, they notified the radiological staff and the VTA primary operator. The Operator arrived and noted that the radiation shielding door was open. He then stopped the test. The shielding door was closed and the test resumed. Four personnel had been previously working in the VTA area at the time of the area monitor alarm. Film badges were processed and fortunately, no employees had received any exposure from this event. An undocumented modification to the wiring to accommodate a special test had been miswired to dewar \#8 contacts instead of dewar \#7 contacts, so the dewar \#7 interlocks were bypassed. This is why the operators did not realize that the shielding door was partially open during the test. The wiring was returned to proper positions. A safety stand-down was performed. A procedure was written for performing interlock alterations when special tests are needed (ORPS ORO--SURATJNAF-2001-0003).

On August 14, 2001, an accelerator SRF component lifting device (a hoist) failed when its chain broke and the hoist fell from its own weight. The special hoist that Jefferson Lab designed and fabricated was located in the Production Chemistry Room, Building 58 (Test Lab). The hoist was installed for handling larger accelerator SRF components of up to $90 \mathrm{~kg}$ (200 pounds) maximum weight. As the chain broke, the hoist fell approximately four $1.2 \mathrm{~m}$ (4 feet) and landed on the lid of an ultrasonic tank. A Production Chemistry Room technician was approximately $1.8 \mathrm{~m}$ (6 feet) away from the hoist at the time of the hoist chain failure. The technician was not injured by the falling hoist. Preliminary review tentatively noted that the hoist's chain and the chain sprocket did not align properly. This misalignment possibly stressed the chain, by placing a bending action on the chain, and may have been the direct cause of the hoist chain failure (ORPS ORO-SURA-TJNAF-2001-0004).

On Monday, December 10, 2001, an Accelerator Division technician (a journeyman electrician and mechanical fabrication technician) received an electrical shock at approximately 1345 hours in Building 98. The electrical shock resulted when the technician clipped a welding machine's spring-loaded ground lead to the worktable (the 
worktable is grounded to the Building 98 steel frame). Earlier in the day, the technician had repaired the $480 \mathrm{~V}$ welding receptacle wiring (which feeds the welding machine) following the discovery of a welding machine problem. This repair resulted in a wiring mistake that the following electrical circuit verification did not disclose, resulting in the electrical shock. The exact mechanism of the shock path that caused the electrical shock is under investigation. The technician was sent to the Jlab clinic and was referred to an outside physician. The technician received medical treatment (a prescription pain reliever) and medical work restrictions for several days. No permanent physiological injury is expected from this electrical shock event (ORPS ORO--SURA-TJNAF-20010005).

At about 1340 hours on Monday, Feb. 4, 2002, an Accelerator Division, Free Electron Laser Instrumentation \& Controls Group supervisor received an electrical shock to her left hand. The shock resulted when her index finger came in contact with a $50 \mathrm{M}$-ohm resistor that was connected across two terminals of an energized 10 microfarad/4,000 V capacitor. She and an Operations Group technician were performing checks of new ion pump power supplies because some similar units had a history of workmanship problems. The supervisor said that she had briefly left the immediate work area and when she returned to the work area was unaware that the power supply was still energized. The supervisor received an electrical shock to her left thumb and index finger. She was taken to Jefferson Lab Medical Services and was referred to the Riverside Regional Medical Center's emergency room. She received medical treatment for second degree burns to her thumb and second and third degree burns to her index finger. The supervisor had ten restricted work days, and volunteered to brief personnel about the event (ORPS ORO-SURA-TJNAF-2002-0002).

A rented temporary cooling tower at Jlab suffered a major loss of function when the unit's fan blades were ejected. There were no personnel injuries or significant damage to other Jlab structures or equipment. The temporary cooling tower was rented in December 2001 as a result of a cooling water piping failure at the accelerator site. A single replacement tower was planned and ordered to be installed. The temporary cooling tower is located between Bldg. 102, the End Station Refrigerator Building, and the Experimental Hall A truck ramp. Lab staff noticed debris upon return to the work area on the morning of July 5, 2002, following the 4th of July holiday. A subsequent review of temperature recording information indicated that the event occurred about 1045 hours on July 4, 2002. The fan unit's gearbox had torqued and pulled two bolt fasteners through the base plate. All of the fan blades had sheared off at the fan hub. The fan shroud, screen cover and blades were found around the fan unit. Some fan blades were an estimated $36 \mathrm{~m}$ (40 yards) from the fan. Fortunately, no personnel were at that location when the fan blades failed (ORPS ORO--SURA-TJNAF-2002-0004).

A subcontract electrician at Jlab discovered on July 24, 2002 that a lockout/tagout device, that the electrician had installed a few weeks earlier, was not in place when he returned to that area to perform work involved with that circuit, which had since been energized. It is believed that the lockout/tagout device (designed for use on a double breaker), that had been used to close off two adjacent single circuits, had fallen off the panel. The LO/TO 
device was not a perfect fit to the equipment being locked out and may have fallen off if it was brushed by passersby. There was no injury from this incident due to the diligence of the electrician who verified the circuit status before performing any work (ORPS ORO--SURA-TJNAF-2002-0005).

At about 1800 hours on Tuesday, April 29, 2003, subcontractor electricians were beginning work on the Test Lab high bay lighting replacement. The overhead crane was moved without observing the position of the hook. The crane hook struck an equipment cabinet where two electrical conduits were severed, and continued moving until striking stacked radiation shielding blocks and an installed radiation monitor. The subcontract electrician (Worker \#1) who was operating the crane had moved the crane without observing the crane hook's position. Worker \#1, who was a qualified Jefferson Lab crane operator, stated that he had found the crane in the same position that he had left it in the previous night. He stated that he assumed that the crane hook was in the same position and started moving the crane without checking the hook location. The second subcontract electrician (worker \#2), who had been assigned to be the safety watch, stated that he left the area of the crane hook and was preparing to load materials. The crane hook struck the equipment cabinet and extensively damaged two conduits that were on the cabinet's top. One conduit contained eight control cables and the second conduit contained a 120 Volt, 20 Ampere control power circuit. The crane hook then struck several masonry shielding blocks and an installed radiation monitor for the Test Cave located below. No hazardous or radioactive materials or radiation producing activities were associated with this event (ORPS ORO--SURA-TJNAF-2003-0001). No one was injured in this event, but perhaps the controls put in place after the March 28, 1995 crane hook event above have either eroded or have not been as effective as possible.

A Jlab technician was exposed to a mixture of sulfuric and hydrofluoric acids when he was removing an acid container from a Test Lab Building electropolishing cabinet. The event occurred on Friday morning, June 13, 2003. The electropolishing cabinet is used for accelerator superconducting radiofrequency component processing. Investigators believe that the worker inadvertently wiped his unprotected face with his protective gloves still on his hands; the gloves had contacted droplets of the acid mixture while he was removing the full container. The worker, after rinsing off at a safety shower and applying calcium gluconate, drove to the Jefferson Lab Medical Services building, where he received initial treatment for facial skin blistering. The worker had no respiratory problems or symptoms at this time. The worker was transported by ambulance to a local hospital for further evaluation on Friday morning. He was discharged from the hospital on Friday afternoon and returned to work on Monday June 16, 2003 without any work restrictions. There was no evidence of any skin blistering upon his return to work on June 16th. It was identified that no follow-up medical evaluation was needed (ORPS ORO--SURA-TJNAF-2003-0002).

At about 1430 hours on July 23, 2003, the crane was positioned for a lift of a vacuum pump to an adjacent cold box. The crane control box for radio-controlled crane operation was hanging from the strap around the crane operator's neck. The crane began unplanned - movement southward, where the crane hook contacted and damaged several 
water pipes before the crane came to rest. The pipes were dented and bent by the impact. There were no personnel in this area and there are no injuries associated with this event. Property damage to the piping has been estimated at $\$ 2 \mathrm{k}$. It is not clear to Jlab management if there was a crane operator error or a crane malfunction that resulted in unplanned crane movement (ORPS ORO--SURA-TJNAF-2003-0003).

Although the Jlab has not operated as long as other accelerators, it has suffered some of the same types of events. Mechanical and electrical equipment fires, personnel receiving electrical shocks, a person struck by a falling object, a hoist failure, unplanned crane movement events leading to equipment damage and hazards to personnel, fan blade ejection, employee chemical exposure, and people in areas being exposed to radiation or RF energy. Jlab had a ferromagnetic object (a camera tripod) drawn by magnetic fields.

\subsection{Relativistic Heavy Ion Collider}

Much of this description came from the facility web pages at the bnl.gov web site. The Relativistic Heavy Ion Collider (RHIC) began operation in the summer of 2000 (Trbojevic, 2001), after a ten-year planning and construction interval. The RHIC total cost was $\$ 616 \mathrm{M}$. The accelerator is located at Brookhaven National Laboratory (BNL) near Upton, New York. The RHIC is supported by several other facilities at BNL, including a tandem Van de Graaff accelerator to initially accelerate ions to $40 \mathrm{MeV}$, a 853-m Tandem-to-Booster beamline, a linear accelerator from this beamline to accelerate ions into the Booster synchrotron. The Booster imparts more ion energy and then sends the ions to the Alternating Gradient Synchrotron (AGS), where the ions are accelerated to $99.5 \%$ of the speed of light, hence the 'relativistic' description. Then the ion beam is taken down to another beam line called the AGS-to-RHIC (ATR) transfer line. The transfer line has a " $Y$ " intersection, where beam ions directed left enter the ' $\mathrm{X}$ ' or 'blue' clockwise ring of the RHIC and beams directed right enter the ' $y$ ' or 'yellow' counterclockwise ring of the RHIC. Both RHIC rings are $3.8 \mathrm{~km}$ circumference. The RHIC accelerates the ions even faster, to $99.995 \%$ of the speed of light, and controls countercurrent beam interactions at up to six interaction points between rings. The RHIC can accelerate heavy ions such as silicon and gold. Some experiments have included deuterium-gold collisions.

Some descriptions and industrial safety information from the AGS and BNL are given here. A preliminary fire protection analysis in 1988 of the AGS Target Halls indicated that there are loss potentials over $\$ 1 \mathrm{M}$. The fire protection professionals noted a shift by the experimental physics community toward large, high valued detectors. The accelerator experiments typically use flammable gases, combustible signal cables, plastic scintillating materials, and are closely arranged in the experiments. The Target Halls are equipped with conventional heat or smoke detection at ceiling level. Early fire detection is not expected due to the very high ceilings (over $21 \mathrm{~m}$ ). Localized spot detection is provided in some areas. The building's combustible Class II roof is not expected to be involved in a fire, unless large quantities of combustibles burn (as in a trailer). Strict housekeeping policies have been adopted to reduce the risk. The number of trailers have 
been reduced inside the building. Local protection has been provided over and above localized concerns (BNL, 1988). A VESDA system was scheduled for installation in the target halls in stages over a period of a few years.

The RHIC and AGS did not have individual listings in CAIRS, so no safety performance plots were available for just the accelerator portions of the BNL site. Any plots would have included the nuclear fission reactors and other operations being conducted at BNL, and as such are not strictly comparable to the other figures presented in this report, so they have been omitted here.

From 1991 to 2003 there were 57 AGS/RHIC ORs in the DOE ORPS database. Some of these were environmental issues, such as transformer oil leaks, some were procedural noncompliances, some were unplanned halon fire suppressant gas actuations, and some events were equipment failures that did not endanger any personnel. The industrialsafety related events on the AGS and RHIC are summarized below since the AGS is connected to the RHIC.

A Westinghouse molded case circuit breaker is installed in the 480 Vac input line of a $300 \mathrm{~kW}$ power supply as line protection. A line contactor is used for operational on/off control. The Bakelite handle of the circuit breaker had a metal extension to aid the manual operation. The extension and part of the Bakelite handle broke off on April 5, 1993. The next day, a technician tried to reset and close the breaker by manually operating the remaining part of the handle. He received a shock in his right hand to his grounded left hand. A metal rod was found to be exposed by the broken handle. Voltage at this rod was measured to be $287 \mathrm{Vac}$ to ground. The technician was not seriously injured; he was not burned or have any other injuries. The threaded rod in the breaker handle was found to be connected to the load side B phase of the breaker. The breaker was reassembled with a new handle that did not have a metal rod (ORPS CH-BH-BNLBNL-1993-0009).

On January 23, 1995, two electricians needed to identify a circuit breaker that supplied power to an RHIC building addition that had been completed in 1993. The two electricians requested help from two towerline electricians who had a generic work permit for working on live lines. The four electricians looked at the breakers and decided that the breaker having a new-looking feed cable would be the one that powers the building addition. One of the towerline electricians began to remove the back cover of the distribution panel. The towerline electricians were only in the area to drop off some equipment and they were not fully prepared with proper equipment to perform work around live lines. The towerline electrician used a non-insulated screwdriver to remove hexagonal screws, and then a screwdriver blade in an electricians knife to remove a slotted head screw. He inadvertently dropped the knife, which slide into the distribution panel via an adjacent cable tray. The uninsulated knife caused a short circuit that ignited an electrical fire. The four electricians were able to move away from the fire but were in a dead end corridor. Flames obstructed their primary path of egress and a deadlocked door obstructed their secondary path. The towerline electrician kicked open the 
deadlocked door, injuring his leg in the process. The four electricians escaped the fire unharmed (except for the one man's injured leg). Causes were inadequate labeling of electrical equipment, inadequate electrical drawings, and electricians not following procedures of working with proper tools and equipment (ORPS CH-BH-BNL-PE-19950002).

On March 27, 1995, a low-background gamma survey was being performed on equipment near the B Test Beam. The owners, who are called AGS Users, planned to return this equipment to their university. The measurements showed that levels on the equipment were about $20 \mathrm{microrem} / \mathrm{h}$, which was about twice background. Ten days prior to finding the high radiation level, on March 17, 1995, the B5 target broke during a high intensity run and the failure was thought to be due to repeated thermo-mechanical stresses from the pulsed beam. Following this, it was discovered that the gate leading to B5 target was contaminated at about $80,000 \mathrm{dpm} / 100 \mathrm{~cm}^{2}$, but the nearby B Test Beam Area was found to be clean. The levels outside this gate had been 200 to $1000 \mathrm{dpm}$ per $100 \mathrm{~cm}^{2}$ routinely due to deposition of short-half-life (20 to 30 minutes) air- activation products since early January, and the location had been roped-off as a Contamination Area. A plastic barrier had been installed to prevent significant air-flow from the beamline toward experimental areas. Periodic-air and daily-contamination surveys had been and continue to be taken in order to ensure contamination is not spreading. The B5 target was replaced, and the surface areas outside the gate were cleaned-up to the prior lowlevels of air-activation products. A second barrier was installed at the gate to help prevent the spread of short-lived (i.e., several months) residual contamination from the broken target. The normal, high residual dose-rates inside the unoccupied target area are such that decay was determined to be the best method of contamination reduction as opposed to a clean-up for this particular area. A replacement target was installed on March 30. Use of the target was allowed after establishing a lower intensity-limit and installing five-interlocking thermocouples on the platinum target to help ensure the new B5 target would not be damaged. Since Users' equipment is not normally activated, it was immediately thought on March 27 that the radiation from the equipment was due to contamination from the March 17 incident. However, the standard, daily beta-emitter surveys taken since March 17 did not show beta contamination on this equipment. Subsequently, a nuclide analysis indicated that the radiation from the equipment was due to the decay of 95-day Osmium-185 and 53-day Beryllium-7, which do not emit beta radiation. The $20 \mu \mathrm{rem} / \mathrm{h}(200 \mathrm{nSv} / \mathrm{h})$ from osmium- 185 and beryllium-7 is not readily distinguishable from the 'sea' of gamma radiation normally measured in the experimental areas. Levels are typically 2,000 to $50,000 \mathrm{microrem} / \mathrm{h}(20$ to $500 \mu \mathrm{Sv} / \mathrm{h})$ during running periods, and 300 to $400 \mathrm{microrem} / \mathrm{h}$ ( 3 to $4 \mu \mathrm{Sv} / \mathrm{h}$ ) when all beam lines are off. The four Users who owned the equipment were examined in the whole-body counter and found to have 50 to 90 nanoCuries (1,850 to 3,330 Bq) of Osmium-185 and Beryllium-7 in their bodies. This may be interpreted as a committed dose of up to 4 mrem $(40 \mu \mathrm{Sv})$ depending on the internal dose model used to interpret the whole-body counting results. Other personnel involved in the target replacement and clean-up were whole-body counted and no intake was found as of the morning of March 28, 1995. Whole-body counting continued past March 28, and about 30 individuals had been counted as of April 12. No significant body burdens were detected (ORPS CH-BH-BNL-AGS-1995-0002). 
At about 1030 hours on June 14, 1995, a 0.43-mm-thick Kevlar and 0.127-mm-thick Mylar window on a vacuum decay-tank in the B5 beam line in Building 912 broke suddenly. The window measured $0.86-\mathrm{m}$ by $1.87-\mathrm{m}$. This failure caused air to rush violently into several hundred cubic feet of space inside the decay-tank. No one was injured, but experimenters reported the explosion noise and dust cloud to the main control room. About $\$ 20,000$ in damages were initially estimated to experimental detectors downstream of the window and tank. The labor to repair the equipment was initially estimated to be four person-months. The Kevlar (for strength) portion of the window was torn free of the frame on both sides, the bottom, and $40 \%$ of the top, and the Mylar (vacuum seal) portion of the window was obliterated. The failure occurred quickly. The tear in the Kevlar, once started, went around the window quickly until the window flapped open allowing a large slug of air to enter the chamber bringing with it the adjacent detector equipment. A $0.8-\mathrm{mm}$ speck of metal or dirt was found jammed into the Mylar frame on the low-pressure side of the window. From the inspection it was clear that when the window is under pressure, this speck rested on the metal frame and was pushed into the Mylar and Kevlar. The tear is $0.8-\mathrm{mm}$ away from the speck. It is possible that the speck caused a localized increase in stress that started the tear. Noted also was that the tear in the Kevlar went about the same distance on either side of the speck. Further analysis of this area is being performed. The window was assembled in the middle of Building 922, which is a mechanical equipment shop. There are metal cutting and machining tools in proximity to the assembly area. Because of the size of the window, an overhead crane must be used in order to lower the window clamp onto the window plate. These operations could have resulted in the speck of debris, which was found under the window as previously described. All other Kevlar windows have been removed from service at AGS. There was some concern that the failure might also have been age-related. The solution to longer lasting windows (i.e., decades) appears to be in using a greater number of Kevlar yarn fibers per square inch in the weave. This window used Kevlar fabric with about $50 \%$ fewer fibers per 645 square millimeters over that used by other accelerators even though the window thickness could be the same (ORPS CHBH-BNL-AGS-1995-0003).

The Pulsed Power Group Leader entered the H-10 house on June 26, 1996 and caused an electric discharge to occur by pushing an expanded metal cabinet wall approximately $1.5 \mathrm{~mm}$ to gain clearance. The metal wall touched live conductors; the capacitors in the power supply discharged and melted a hole in the expanded metal wall about $25 \mathrm{~mm}$ in diameter. Molten aluminum was sprayed onto the eyeglasses of the Group leader. Four persons were present and were exposed to the electrical arc flash, but no one was injured. The Bump Power Supply enclosure will be re-designed and modified to result in an improved electrical hazard barrier, and prevent inadvertent contact with the high-current conductors and stored energy (ORPS CH-BH-BNL-AGS-1996-0001).

On December 19, 1996 an experiment user from Experiment 896 received an electrical shock while assembling a detector near the beam line. The user was holding a flexible metal vacuum hose attached to a vacuum pump and received a shock when he attempted to connect the hose to the grounded magnet vacuum chamber. The pump skid was 
plugged into a $208 \mathrm{~V}$ ac outlet. The user reported the shock was not severe and he did not experience any symptoms of injury. He continued to work. Investigation revealed that the pump skid plug was missing a connection to the neutral pin in the connector. The extension cord that was attached to this improperly-wired pump cord was also improperly wired. It was a 4-wire extension cord with no ground connection to its connector shell. The AGS standard is to use 5-wire for a 3-phase extension cord and to have the ground on the cord's connector shell. The AGS policy for proper grounding was not being enforced (ORPS CH-BH-BNL-AGS-1996-0004).

On April 12, 1997, an AGS HP technician was performing a routine radiation survey near the B5 beam line. He discovered the southeast corner of the orange radiation barrier had been taken down. This allows access to the top of the shield blocks near the B5 line. This area is a High Radiation Area With Beam On and was posted as such. The fence was re-established. An inquiry was begun. An initial report by an E935 experimenter is that another E935 experimenter, who just left BNL for the west coast, had cut the fence down to more easily access detectors in the beam line. When this was learned, the B5 beam line was shut down and an investigation begun. The beam line remained shut down until further notice by AGS management. An experimenter willfully circumvented safety rules at AGS and placed himself - and his colleagues - in jeopardy of significant radiation exposure. On several occasions, the experimenter removed straps from a "High Radiation Area With Beam On" barrier and created an opening in the fence in order to improperly access detectors in the B5 beam line. In addition to willfully violating AGS safety rules, the experimenter allowed the opening in the fence to remain after he left the beam line. Thus, the experimenter created a potentially hazardous situation for others whenever beam was on. The User's March 1997 dosimeter badge was located after a collaborator searched a shared on-site apartment on April 13 (this was another poor practice to take the dosimeter to the apartment and to not turn it in for processing at the end of the time interval). The badge was immediately processed and indicated $43 \mathrm{mrem}$ $(430 \mu \mathrm{Sv})$. A phone interview with the E935 user in question on April 14, 1997 at 1400 hours established the following: The user stated that he obtained a Visitor's badge and was assigned an escort for the period March 13 to March 25, 1997. He stated that he cut the fence straps on multiple occasions. After creating a way through the fence, he stated that he left it open since he knew he needed to return. On one or more occasions of return, he would find the barrier repaired. The User stated that he always wore his TLD badge when he broke through the fence. He stated that he always checked to see if the gate to B5 beam line was open before crossing the shield top and reaching into the beam line. He felt this was the easiest and safest way to access his detectors. He acknowledged that he knew the rules about not violating a radiation barrier. He felt that this barrier was not a radiation barrier. He stated he kept his March badge with him until the day he left, April 11, since it was only a few more days past the due date of April 5. The User's statements appear to be contradictory in that he checked to see if the B5 gate was open before crossing the barrier, yet he felt the barrier was not a radiation barrier. AGS User Training makes users aware that they must enter beam lines through a gate because gates have interlocks that inhibit beam. Thus, an open gate always eliminates the potential for an in-beam radiation hazard. AGS User Training emphasizes one "Golden Rule" which is "never climb over or defeat a radiation barrier or fence." Users are 
explicitly tested on this rule, as was this User. The user in question correctly answered several questions on his exam, which dealt with barrier violation, but in practice he did not follow the rule. The user is indefinitely debarred from entry to the BNL site (ORPS CH-BH-BNL-AGS-1997-0002).

On June 2, 1998, a Controlled Access period began at about 0830 hours for the AGS Ring. Controlled Access requires personnel to sign-in and sign-out at a single gate to the AGS Ring. As lunchtime approached, the personnel began to stop their tasks and sign out to go to lunch. The Operations Coordinator conferred with the Gate Watch person. Believing that all persons were out of the ring, the Operations Coordinator placed the machine on a beam-enabled state, which interlocks the gate and prevents entry. This new state allowed the Gate Watch to leave for lunch as well. The Operations Coordinator did not have a sweep of the AGS ring performed since there was no intention to initiate a beam. When the machine was switched to beam-enabled state, a technician still working in the ring noted the lights dimming as a sign of the enabled state and he reported to the gate since he believed that a beam was going to be started. The gate watch did not understand that some other employee had erroneously entered a name in the location where the technician would sign out on the log sheet. The name had been crossed out, but at first survey of the form the gate watch believed that all persons were accounted for. The Operations Coordinator was counseled that the safety procedures are to be followed explicitly and are not open for interpretation or deviation for operational convenience without a specific review and management consent (ORPS CH-BH-BNL-AGS-19980003).

On July 16, 1998, four technicians were riding an electric cart. The cart exited a dirt road and slid sideways upon entering the paved surface, causing the cart to go up on two wheels. One of the occupants fell from the vehicle. The fallen cart occupant complained of back pain, and he was sent to a local hospital as a precaution. The injured person was transported to a local hospital for examination. He reported that the doctor indicated that he had a sprained back and bruised soft tissue. He was then sent home. The employees' behavior in operating the vehicle in such an unsafe manner (taking it off the paved road of the RHIC construction site and speeding) is not condoned by RHIC management. The employees involved were suspended for 10 working days without pay (ORPS CH-BHBNL-BNL-1998-0018).

On November 5, 1998, an AGS Mechanical Services Technical Supervisor (MSTS) entered the AGS Ring, which is a High Radiation Area, after discussion with the Maintenance Coordinator and the Associate Head of the Accelerator Division, to look for an argon gas leak in the Radiation Loss Monitor System. The AGS was in a shut down condition and the MSTS entered this locked area after his self-reading dosimeter and training record were checked by the gate watch. The MSTS discovered the location of the leak and took it upon himself to repair the leak without proper work planning. Upon completion of the repair, the MSTS noticed a posting in the area indicating 7,400 $\mathrm{mrem} / \mathrm{h}$ $(74 \mathrm{mSv} / \mathrm{h})$ at $30 \mathrm{~cm}$. He immediately left the AGS Ring and reported to the Maintenance Coordinator. His self-reading dosimeter was pegged at 200 mrem $(2 \mathrm{mSv})$. A Radiological Control Technician then surveyed the area where the work was 
performed. The survey indicated that the radiation level in the area was $5,000 \mathrm{mrem} / \mathrm{hr}$ $(50 \mathrm{mSv} / \mathrm{h})$. Based on the results from the MSTS's dosimeter badge and on a subsequent dose investigation, the exposure of the MSTS was better estimated to have been $180 \mathrm{mrem}(1.8 \mathrm{mSv})$ due to this unplanned task. Since the daily AGS Administrative Control Limit is 100 mrem $(1 \mathrm{mSv})$, the event was further investigated as an off-normal occurrence. The AGS Policy was not clearly defined and as a result not enforced. This task was not classified as a job. As a result, it was unclear to the Accelerator Division leadership chain that this task needed formal review for hazards. Thus, the AGS procedure titled "Enhanced Work Planning," was not invoked prior to authorizing a job. A contributing cause is stress due to a tight schedule. Investigation revealed that the maintenance coordinator, the MSTS, and the Associate Head of the Accelerator Division had all discussed finding the argon leak and had decided to turn up the argon flow so that the hissing of escaping gas would allow quick identification of the leak. But they did not enter the task into the work control system. As a result of this event, the staff now has to be equipped with pencil dosimeters and alarming ("chirping") personal dosimeters when working in radiation fields, the staff will be retrained on using the work control system, and the management involved will be counseled for planning a job in a high radiation field and not performing a hazard analysis (ORPS CH-BH-BNL-AGS-1998-0004).

At 0849 hours on May 11, 1999, gas pressure in the RHIC cryogenic system had to be manually relieved to lower the pressure. The Cryogenic Shift Supervisor determined that a $7.6 \mathrm{~cm}$ (3 inch) manual ball valve should be opened to reduce pressure below the setpoint for automatic venting. Because of the unique set of circumstances, the initial startup of the systems and the time pressure to vent the gas, personnel were not fully aware of the potential hazards and the controls to minimize hazards. In the process of opening the manual valve the Operator slipped and got partially in the path of the gas exiting the valve. The valve is about $1.27 \mathrm{~m}$ (50 inches) above the ground and vents horizontally. A large break-away torque was required to begin to open the valve. The noise from escaping gas caused the Operator to reclose the valve and quickly obtain hearing protection. When he re-opened the valve, the required torque was much less than he expected and he lost his balance as the valve quickly and easily re-opened. The Operator slipped into the escaping gas stream. He was propelled into a nearby helium storage tank by the high velocity exhaust gas, causing a minor laceration to his head. At 0906 hours he was transported by ambulance to the hospital for observation. Following examination and treatment, the Operator returned to work by 1500 hours that same day. A second Operator was at the scene during the valve operation in order to satisfy the twoperson staffing rule. The second Operator called for help on the radio and a third Operator from the Cryogenic Control Room (CCR) went to the scene. Radio communications were poor due to the high noise caused by the venting helium gas. The third Operator had the CCR call for an ambulance. Meanwhile, the second Operator helped the victim away from the scene. The valve was shut at 0915 hours when the gas pressure was stabilized. After the event, a vent pipe extension was installed on the $7.6 \mathrm{~cm}$ ball valve that will direct the escaping helium to a point $\sim 4.5 \mathrm{~m}$ above the ground where any effects to the operator will be minor (ORPS CH-BH-BNL-BNL-1999-0012). 
On February 10, 2000, a technician cutting electrical cables with an insulated cable-cutter saw a flash of light when he cut through a cable. The employee was cutting and removing cable in the Slow Extracted Beam experimental area encompassed by Building 912. This area is under long-term renovation, and has not been operating since September 1999. The employee was wearing leather gloves but was not wearing safety glasses. He was brought to the Clinic after complaining about a blind spot in the corner of his right eye. After an examination showed no damage to the employee's eye, the Clinic released the employee back to work without restriction and the employee returned to work at 1600 hours. Cable cutting was stopped and the power to these cables and lines were retraced. The electrical flash was determined to be from two phases of a 208Y/120Volt, $60 \mathrm{~Hz}$ power source that had not been isolated. Work planning was not adequate to protect the workers in this case (ORPS CH-BH-BNL-AGS-2000-0002).

At 0050 hours on October 4, 2000, a Collider-Accelerator Support (CAS) Watch entered Building 930 for a routine check of equipment running at Linac. He found a smoke-filled building and pulled a fire-alarm box. He went outside and noticed no alarms, nor did the Fire Department respond. He went back into Linac Control Room in Building 930, a location where he noticed no smoke and called extension 911. The on-duty Fire/Rescue Officer told the CAS Watch to pull another fire-alarm box and to go out to the road to guide the responding unit. The CAS Watch pulled a box near the Linac Control Room and left the building. This pull box caused the discharge of the halon fire-protection system and activated local audible alarms, however this pull box also failed to alert the Fire Department. First-arriving Fire Department staff found a moderate smoke condition. They searched the facility but did not find the source of smoke. Linac personnel arriving at 0120 hours found a damaged Motor Control Center (MCC) with burned trash-bag material in front of the MCC panels. The fire burned itself out and smoke was vented without incident. The area was sprinkler protected but the fire heat was insufficient to actuate the sprinklers. The cause of the electrical failure was likely a high resistance in the supply from the buss. The resulting heat from the resistance most likely damaged the insulation of the conductors within the compartment. Since there was no indication of arcing to compartment walls, a phase-to-phase short would have occurred and fuses destroyed. The resulting arc over would have over-pressurized the compartment with the flash. There were bulges in the door indicating overpressure. The shower of sparks would have ignited the plastic bag directly under the compartment. Short-circuit current was limited to about 30,000 amperes for this MCC by upstream over-current protection. The fire alarm panel was also investigated. A 2-amp fuse blew on the fire alarm panel in Building 930. The cause of the blown fuse is not clear. It may have been blown since the last time PM was performed, or blew coincident with this event. With this particular fuse blown, it puts the fire alarm panel in a "sleeper" mode. That is, for this type of failure, there is a loss of all alternating current and backup direct current power to a portion of the fire alarm panel. Better preventive maintenance will be performed on electrical distribution systems, and upgrades to fire alarm panels will be scheduled to remove units that have these single point failures (ORPS CH-BH-BNL-AGS-2000-0004).

On October 18, 2000, a technician, accompanied by an engineer, was checking the status of a beam instrument package near a beam pipe in an accelerator tunnel. This instrument 
package had been recently relocated to this area. The technician, on his knees during the inspection, was preparing to stand up. He put one hand on the instrument package cabinet and the other hand on a column that supports the beam pipe. His right hand, on the beam pipe support, contacted a cut coaxial cable causing a shock at approximately 1155 hours. The technician reported to the Clinic, was examined and given an electrocardiogram; nothing abnormal was noted. He was observed to have a small wound on his right hand where the cable contact was made and a small contusion on his shoulder from backing into a wall in reaction to the shock. After being examined at the BNL Clinic, he returned to work. The cut cable was energized at about $6 \mathrm{kV}$ and was not related to the instrument being inspected. It was quickly determined that the cut coaxial cable was connected to a DC power supply for two beam line ion vacuum pumps. The power supply is located on the second floor, remote from the ion pumps. The output voltage varies as the direct current varies. The DC output is about $1 \mathrm{~mA}$ at $5 \mathrm{kV}, 100 \mathrm{~mA}$ at $2.5 \mathrm{kV}$ and $200 \mathrm{~mA}$ at 21 Volts. The direct cause was an improperly exposed conductor. Previous work had incorrectly identified matching equipment powered from a common source. There was incorrect post-work testing to verify the final configuration of the wiring and confirm ion pump operability (ORPS CH-BH-BNL-AGS-2000-0005).

On August 3, 2001, at 1259 hours, a smoke detector alarm from building 928 (the Motor Generator Building) was received at the Brookhaven National Lab (BNL) firehouse. This alarm was followed by other fire detection alarms in the building. The BNL Fire/Rescue Group responded and found smoke coming from the building. The fire alarms caused the evacuation of building 928 and a contiguous building 929 . When the fire department arrived, it was assumed that there was a working fire in the building. In accordance with the BNL Emergency Plan, the Incident Commander declared an Operational Emergency at 1325 hours. On receipt of the alarm, motor-generator (MG) Operators, present in building 928, returned to the MG set control room within the building to determine the cause of the alarm and the status of the MG set. This MG set powers the AGS main magnets via transformers, rectifiers, switches and filters. The MG set was found tripped, the electrical motor brake was on and there was no smoke visible in the MG room. The MG room is adjacent to the MG control room. The two operators entered the basement to determine the cause of the alarm. Upon seeing smoke in the basement, they called the accelerator complex Main Control Room to report that the alarm was real, verified that there were no injured personnel in the building and evacuated to await arrival of the BNL Fire/Rescue Group, who arrived at 1303 hours. Firefighting personnel connected their fire truck pumper to the building fire-standpipe and pressurized the system in preparation to fight a fire. The MG set operators entered the building with firefighters to determine the source of the smoke. Upon entering the basement, they found a cracked fire-standpipe drain line, causing water to spray near equipment. The standpipe was depressurized, and water and electrical power to the building were isolated. When the building electrical power was isolated, the MG set forced air ventilation ended. Smoke began to collect in the MG room. Eventually, when the smoke cleared, the operators visually inspected the internals of the MG set through view ports. Debris was visible on the bottom of the stator at the exciter (south) end of the generator. The Fire Department did not have to apply water. The AGS shut down, and consequently the RHIC also shut down. Investigation revealed that the recently 
refurbished MG set had been given a new retaining ring but that the ring was inadequate to handle the service stresses. A ring with $65 \mathrm{ksi}$ yield strength was used, when in reality the service stress for the retaining ring is between 95 and $130 \mathrm{ksi}$ for the MG set. The retaining ring failed after eight weeks of operation. When the retaining ring yielded, ring material contacted the stator windings and iron core, which resulted in a catastrophic failure of the retaining ring, the shorting ring, and the stator windings. The consequent electrical arcing and hot debris caused the smoke that actuated the building fire alarm. No one was injured in this event (ORPS CH-BH-BNL-AGS-2001-0001).

On May 15, 2002, the Mechanical Service Group (MSG) released approximately 1 liter of water to the floor of the AGS Ring (Building 913) in the location of a water-cooled magnet during maintenance repair of a cooling water hose. Radiological Control Technicians (RCTs) covering the job measured beta contamination at a level of 6,000 dpm on the surface of the sole of the shoes of the MSG Technician as he exited the Radiation Area. The magnets and magnet bus in the AGS are supplied by the Cooling Tower 1 water system, it provides water at about 200 psi. In the past, the water in this system has not been significantly activated. Approximately four months ago, at the end of the RHIC run in which this line was last in service, the magnet appeared to have developed a cooling water leak. The magnet's water system was valved off to prevent any release of water, and the small amount of water remaining in the magnet essentially became standing water at atmospheric pressure. The cooling system was taken out of service, and repair of this leak was to be performed at a future time. On May 15, 2002, at approximately 10:30 hrs, the Technical Supervisor (TS) and a Technician from the MSG along with two RCTs entered Building 913, through the North Gate. The MSG personnel were to diagnose and repair the water leak on the magnet. The RCTs performed a survey prior to the MSG personnel entering the area. The MSG TS inspected the magnet and determined that a water hose fitting was leaking. The TS left the magnet area and returned to the North Gate area to fabricate a replacement hose. The TS with one of the RCTs, reentered the area. The TS loosened the hose fitting on the existing magnet cooling hose, releasing approximately 1 liter of water onto the floor. The TS removed the old hose and attached one half of the new hose to the magnet. The TS observed that he was approaching $17 \mathrm{mrem}(170 \mu \mathrm{Sv})$ on his digital dosimeter and left the area so as to not exceed the 20 -mrem $(0.2 \mathrm{mSv})$ dose-limit, which was specified on the radiological work permit used for this job. The MSG Technician entered the magnet area to complete attaching the water hose to the magnet. As observed by the RCT, both the MSG TS and Technician had walked in the released magnet water. As a prudent practice the RCT frisked the two workers for contamination. The sole of one the MSG TS shoes was contaminated at a level of $1500 \mathrm{dpm}$. This shoe was decontaminated and returned to the TS. The MSG Technician's shoes were contaminated at a level of 6,000 dpm. These shoes could not be decontaminated and were confiscated by the RCT. Additional surveys showed that the workers had no additional contamination. The shoes were analyzed; Na24 (14 hour half life) and Be-7 (54 day half life) were found. These isotopes were unexpected in the cooling water; usually the water is activated with short-lived isotopes such as O-15 (half life of 2 minutes). Investigators believed that the water standing in the magnet for several months had become activated by the AGS main ring. The AGS had recently operated with high-intensity protons. The workers were not injured by the 
contamination and they controlled the contamination well (ORPS CH-BH-BNL-AGS2002-0001).

On December 30, 2003 at approximately 0900 hours, a representative of the ColliderAccelerator Department (C-AD) Booster/Alternating Gradient Synchrotron (AGS) Ring Power Supply Systems Group was contacted by personnel at the BNL Warehouse (Building 100) informing them that a flatbed truck had arrived with 7 crates of electrical equipment. The crates were too large to be stored in Building 100 so it was decided that the equipment should be off loaded at a storage location in Building 912. The crates were closely packed on the front of the flatbed truck precluding the use of an overhead crane to remove them. Plant Engineering (EP) Riggers, assigned to the C-A Department, proceeded to off load the crates from the flatbed truck with a fork truck rated at 3.5 tons. The Power Supply Group representatives went to Building 912 to observe the off loading of the crates. They did not have specific information about the individual crates but knew what the total equipment package was. They observed that rigging was proceeding smoothly and left the area. The rigging crew did not have information about crate contents, center of gravity, or weight, and the crate markings did not contain any specific information. Five crates were offloaded without incident. The riggers did not band each crate to the lift truck. When the lift truck, rated at 3.5 tons, was moving the sixth crate, the lift truck encountered a rainwater-filled pothole in the apron area in front of the roll up door. Because of the down-up motion of traversing the pothole, the load (a transformer) shifted inside the crate; it was not secured to the crate bottom as is the usual practice of shippers. The rigger, who was guiding the lift truck operator, ran to the opposite side to help steady the load. He realized that his pushing on the crate was having no effect; the crate was very heavy. He backed away and the crate brushed his leg as it fell off the lift truck tines, which were a few inches above the floor. His leg as bruised. The transformer sustained obvious physical damage in the fall. The injured rigger was sent to the medical clinic, the final, small crate was offloaded to allow the delivery driver to leave the scene, and an investigation was commenced. Causal factors were: 1) crates not adequately labeled as to its contents, weight, and center of gravity; 2) crates were not adequately constructed for the contents contained within; 3) the transformer was not secured to the crate bottom by the shipper; 4) the shipping manifest did not have information about crates and contents; 5) the crates were not strapped to the mast of the fork truck for increased stability and security as required in the Plant Engineering procedure on forklift safety; 6) the apron/road surface was in need of repair and condition was concealed by rainwater; and 7) the Riggers did not adequately follow Plant Engineering Forklift Safety procedures in moving the crates. The apparent cause of the incident was inadequate work planning. The rigging crew had limited information about the individual crates they were transferring (ORPS CH-BH-BNL-AGS-20030001).

An important environmental and safety issue arose with the AGS and Brookhaven National Laboratory in 1997 (Ingrassia, 2001). Tritium was found in the ground water. The first discovery was $5 \mathrm{Ci}$ of tritium from the spent fuel rod storage pool at the High Flux Beam Reactor, a fission reactor on site. The DOE terminated the contract with the Associated Universities managing contractor, closed the fission reactor, and wrote a new 
contract that highlighted environmental compliance (Briggs, 2001). With the RHIC nearing completion, the accelerator staff realized that being able to prove environmental compliance would be crucial to continued operation of the AGS and startup of the RHIC. After detailed checks the site for other possible sources of tritium, three sources were found at the AGS. One tritium source was in the earthen shielding near an active beam dump, another source in the earthen shielding due to chronic beam losses on one of the final quadrupole magnets, and one from the earthen shielding near a decommissioned beam dump. Another source was found elsewhere in the accelerator complex, but has not been openly discussed. The active beam dump area had a tritium concentration that was twice the Environmental Protection Agency's drinking water standard, and the quadrupole magnet area had a tritium concentration that was 90 times the drinking water standard. Since rainwater would transport the tritium and activated sodium to the water table, this was an environmental concern and also a personnel safety concern of ingestion and exposure since Brookhaven has always taken its on-site drinking water, and process water, from six on-site wells (Bennett, 2000). Steel, concrete and soil are the primary shielding materials for accelerators, based on experience, economics, and convenience (Chao, 1999). The AGS and RHIC are no exception. Most of the secondary particles, created from shielding material interactions with the primary protons from the beam, are stopped by the shielding. When a high energy secondary particle interacts with soil shielding, the longest lived radionuclides produced are tritium and sodium-22, at 12.3 and 2.6 years, respectively. The tritium and sodium form water soluble compounds that are easily dispersed by rainwater. After these sources of tritium were identified, changes were made to prevent tritium mobilization to the groundwater. For the active beam dump, it was positioned so that it will not inadvertently intercept the proton beam, which will reduce the scattered protons. A gunite cap was placed over the soil above both beam dump locations to prevent rainwater from leaching the tritium and sodium to the water table or the site boundary. For the quadrupole, the beam optics were reworked to prevent beam losses. Loss monitors were installed near the quadrupole and are regularly checked for beam losses in transport. A gunite cap was placed over the soil near the quadrupole as well. The corrective actions for the other trouble spot was not discussed. The accelerator operators have been given watchdog software that will generate an alarm when beam losses are high at critical, monitored locations or during prescribed segments of the acceleration cycles. The operators have been trained to react as required to beam losses and to proactively prevent beam losses where possible.

\subsection{Other High Energy Physics Machines}

There have been several other events at smaller facilities. Brief descriptions of these older events are presented in Table 3. A few other events are described in more detail below. 
Table 3. Operational Events at Particle Accelerators

\begin{tabular}{|c|c|c|c|}
\hline Event date & Event Description & Event Damage & Reference \\
\hline 11/08/1960 & $\begin{array}{l}\text { Two employees were accidentally } \\
\text { exposed to an electron beam from a } \\
\text { Van de Graaff accelerator. }\end{array}$ & $\begin{array}{l}12,340 \text { rem and } \\
1,277 \text { rem to } \\
\text { hands }\end{array}$ & WASH, 1975 \\
\hline $10 / 17 / 1963$ & $\begin{array}{l}\text { While making an adjustment on a } \\
\text { synchrotron, an employee received a } \\
\text { radiation exposure. }\end{array}$ & $\begin{array}{l}\text { Whole-body } \\
\text { exposure of } 3.8 \\
\text { rem }\end{array}$ & WASH, 1975 \\
\hline $07 / 21 / 1964$ & $\begin{array}{l}\text { A hydrogen purifier exploded when a } \\
\text { valve was inadvertently left closed } \\
\text { during a purging operation. }\end{array}$ & $\begin{array}{l}\text { Precooler and } \\
\text { adsorber coils } \\
\text { were torn open } \\
\text { and the dewar } \\
\text { was bulged. }\end{array}$ & WASH, 1975 \\
\hline $01 / 11 / 1965$ & $\begin{array}{l}\text { An employee received radiation } \\
\text { exposure while working next to a } \\
\text { cyclotron vault. }\end{array}$ & $\begin{array}{l}3.6 \text { rem whole- } \\
\text { body exposure }\end{array}$ & WASH, 1975 \\
\hline $05 / 06 / 1965$ & $\begin{array}{l}\text { A fire occurred due to capacitor } \\
\text { failure in an electrical pulsing } \\
\text { modulator of an electron accelerator. } \\
\text { No injuries. }\end{array}$ & $\begin{array}{l}\$ 127 \mathrm{k} \text { in } \\
\text { equipment and } \\
\text { cleanup costs. }\end{array}$ & WASH, 1975 \\
\hline $05 / 22 / 1965$ & $\begin{array}{l}\text { a physicist received radiation to a } \\
\text { finger when the beam shutter on a } \\
\text { Van de Graaff machine opened } \\
\text { without his knowledge. }\end{array}$ & $\begin{array}{l}51 \text { rem to a } \\
\text { finger. }\end{array}$ & WASH, 1975 \\
\hline 07/07/1965 & $\begin{array}{l}\text { An employee thought an x-ray } \\
\text { machine was off and he reached in to } \\
\text { change materials. He received } \\
\text { exposure to his hand. }\end{array}$ & $\begin{array}{l}1,000 \text { rem to } \\
\text { fingers, } 2^{\text {nd }} \\
\text { degree burns. } 12 \\
\text { days lost during } \\
\text { burn recovery. }\end{array}$ & WASH, 1975 \\
\hline $09 / 15 / 1965$ & $\begin{array}{l}\text { An employee was unaware that an } \mathrm{x}- \\
\text { ray machine was on and the shutter } \\
\text { was open; he reached into the } \\
\text { machine to wipe away moisture. }\end{array}$ & $\begin{array}{l}80,000 \text { rem to } \\
\text { fingers; } 100 \text { days } \\
\text { lost time and } \\
\text { amputation of } \\
\text { part of his index } \\
\text { finger. }\end{array}$ & WASH, 1975 \\
\hline 03/18/1966 & $\begin{array}{l}\text { A hydrogen explosion occurred at the } \\
\text { inlet to an adsorber coil of a bubble } \\
\text { chamber when hydrogen flow began. }\end{array}$ & $\begin{array}{l}\text { No injuries, } \\
\$ 11.6 \mathrm{k} \text { damage }\end{array}$ & WASH, 1975 \\
\hline $\begin{array}{l}06 / 06 \text { to } 19 / \\
1966\end{array}$ & $\begin{array}{l}\text { An employee at a university linear } \\
\text { accelerator had an overexposure. }\end{array}$ & $\begin{array}{l}50 \text { rem whole } \\
\text { body }\end{array}$ & WASH, 1975 \\
\hline
\end{tabular}


Table 3. Continued.

\begin{tabular}{|c|c|c|c|}
\hline Event date & Event Description & Event Damage & Reference \\
\hline $12 / 09 / 1966$ & $\begin{array}{l}\text { A rubber cooling water hose for an } \\
\text { experimental magnet ruptured, the } \\
\text { water spray caused a short circuit } \\
\text { across the bus connections. The arc } \\
\text { ignited polyethylene sheeting. }\end{array}$ & $\begin{array}{l}\text { No injuries, } \\
\$ 8.3 \mathrm{k} \text { in damage }\end{array}$ & WASH, 1975 \\
\hline $06 / 14 / 1967$ & $\begin{array}{l}\text { A Van de Graaff accelerating tube, in } \\
\text { its cradle, was being moved by crane. } \\
\text { Workers did not check the four lifting } \\
\text { slings, which had been shackled but } \\
\text { not lashed together. When lifted, the } \\
\text { tube slid out of its cradle and fell } \\
4.5 \mathrm{~m} \text { to the floor. }\end{array}$ & $\begin{array}{l}\text { No injuries, } \\
\$ 10.6 \mathrm{k} \text { in } \\
\text { damage }\end{array}$ & WASH, 1975 \\
\hline $12 / 24 / 1967$ & $\begin{array}{l}\text { A fire began in the compressor trailer } \\
\text { for a bubble chamber facility. The } \\
\text { cause was probably electrical. }\end{array}$ & $\begin{array}{l}\text { No injuries. } \\
\text { Compressors, } \\
\text { piping and } \\
\text { wiring were } \\
\text { damaged, } \$ 15 \mathrm{k} \text {. }\end{array}$ & WASH, 1975 \\
\hline $06 / 23 / 1968$ & $\begin{array}{l}\text { A defective transformer joint failed, } \\
\text { resulting in a fire. The power supply } \\
\text { transformer was damaged. }\end{array}$ & $\begin{array}{l}\text { No injuries. } \\
\text { Damages at } \$ 12 \mathrm{k}\end{array}$ & WASH, 1975 \\
\hline $07 / 24 / 1970$ & $\begin{array}{l}\text { A bomb was exploded at the } \\
\text { University of Wisconsin, fatally } \\
\text { injuring a researcher in the low } \\
\text { energy physics lab. This act was } \\
\text { attributed to student unrest. }\end{array}$ & One fatality. & WASH, 1975 \\
\hline $\begin{array}{l}11 / 17-18 / \\
1970\end{array}$ & $\begin{array}{l}\text { A staff assistant was exposed when } \\
\text { handling Cu targets from alpha } \\
\text { particle irradiation in a cyclotron }\end{array}$ & $\begin{array}{l}\text { finger doses } \\
\text { from } 150 \text { to } 480 \\
\text { rem. }\end{array}$ & WASH, 1975 \\
\hline 08/19/1972 & $\begin{array}{l}\text { An electrical failure in the rotor of a } \\
\text { motor in the main magnet supply } \\
\text { system resulted in a forced shutdown } \\
\text { of the synchrotron. The motor had to } \\
\text { be removed and sent to a shop for } \\
\text { repairs. }\end{array}$ & $\begin{array}{l}\text { No injuries. } \\
\$ 45 \mathrm{k} \text { to repair. }\end{array}$ & WASH, 1975 \\
\hline $01 / 14 / 1973$ & $\begin{array}{l}\text { A staff engineer was electrocuted } \\
\text { during energized trouble-shooting } \\
\text { operations of a high voltage, high } \\
\text { power RF amplifier at a synchrotron. } \\
\text { Attempts to revive the man failed. }\end{array}$ & One fatality. & WASH, 1975 \\
\hline
\end{tabular}


Table 3. Continued.

\begin{tabular}{|c|c|c|c|}
\hline Event date & Event Description & Event Damage & Reference \\
\hline $07 / 15 / 1974$ & $\begin{array}{l}\text { An electrical engineer was } \\
\text { electrocuted when he sustained a } \\
\text { shock from a betatron capacitor bank } \\
\text { and its support structure that were } \\
\text { energized to } \sim 5 \mathrm{kV} \text { from a low } \\
\text { impedance power source. The } \\
\text { engineer was working on an } \\
\text { energized control circuit at the time. }\end{array}$ & One fatality. & WASH, 1975 \\
\hline $11 / 11 / 1974$ & $\begin{array}{l}\text { A graduate student received exposure } \\
\text { from a } 3 \mathrm{MeV} \text { proton beam at a } \\
\text { tandem Van de Graaff generator. }\end{array}$ & $\begin{array}{l}\text { First and second } \\
\text { degree burns on } \\
\text { hand, } 2 \mathrm{E}+6 \text { rads } \\
\text { to } 2^{\text {nd }} \text { degree } \\
\text { area, and } 4.6 \mathrm{E}+5 \\
\text { rads to } 1^{\text {st }} \text { degree } \\
\text { area. }\end{array}$ & \\
\hline $01 / 26 / 1976$ & $\begin{array}{l}\text { Fatigue cracks were found in a } \\
\text { bubble chamber, operations were } \\
\text { delayed for } 10 \text { months. }\end{array}$ & $\begin{array}{l}\text { No injuries. } \\
\$ 75 \mathrm{k} \text { in damage. }\end{array}$ & DOE, 1980 \\
\hline
\end{tabular}

Table 3 presented several types of events: radiation overexposures, fatal electrical accidents, and equipment fires. While these events occurred from thirty to forty years ago, the hazards and concerns are still valid. Other events important to industrial safety are discussed below.

On July 5, 1965, an explosion and fire occurred in the experimental hall of the Cambridge electron accelerator (AEC, 1966). The complex was located on the grounds of Harvard University, and consisted of a laboratory/office building, a cryogenics building, a power building, the experimental hall, and the accelerator tunnel. Three experiments were under way in the experiment hall on the day of the explosion. These were a 500-liter liquid hydrogen bubble chamber, a liquid hydrogen target and Freonfilled counter for photoproduction of mesons from the accelerator beam, and an experiment on elastic photoproduction of $\mathrm{K}$ mesons that used a propane-filled Cerenkov counter and an ethylene filled Cerenkov counter. Other important equipment included the two 100-pound storage cylinders of liquefied petroleum gas (LPG) used to fuel the emergency electrical generator. These two cylinders were mounted not far from the bubble chamber. While operations were in progress, at 0332 hours an explosion occurred at the bubble chamber. Investigation revealed that the inner thin beryllium window (3$\mathrm{mm}$ thick, 241-mm diameter) catastrophically failed, and that window shards and the hydrogen jet from the chamber then almost instantaneously failed the outer beryllium window of the same size. The inner beryllium window was thought to have failed due to 
fracture from excessive mechanical and thermal stresses placed on it by the windowmounting system when cooled to cryogenic temperature; investigators concluded that the inner window had been poorly designed. The failure of both windows allowed about half the contents of the chamber to be rapidly expelled into the room. The most likely ignition source for the vaporizing cryogenic hydrogen was the fracture energy of the outer beryllium window, but numerous other sources of ignition were also present. This deflagration of hydrogen in the air caused an overpressure of about $10.3 \mathrm{kPa}$ (1.5 psig) in the experiment hall. The pressure lifted the roof and then it settled back onto the building. All the personnel injuries were attributed to the first explosion. Roof insulation, a tar-coated fiberboard, was ignited in some locations. Melting tar that fell to the floor also burned in many locations. Combustibles in the room (cables, papers, hoses, fallen roof insulation) ignited near the bubble chamber. Most of the secondary fires were of short duration due to limited combustibles and conditions being unfavorable for continued combustion. Exceptions were fires consuming hydrogen near the bubble chamber, the darkroom, the two LPG tanks, and the propane supply tanks for the Cerenkov counter. Fire near the two LPG tanks sufficiently heated the tanks until they vented. The venting LPG caused a second explosion and an intense gas-fed fire. Fire heat melted the mylar window of the Cerenkov counter and falling roof debris damaged the propane gas manifold. The window and manifold failures were believed to be the causes of propane release; the gas ignited and also added to the fire. The heat caused the six propane tanks in the room to pressurize and lift their relief valves to vent. Fire heat also melted the fusible safety links on the cylinder valves to allow even more venting. The venting propane caused another fierce fire in the room. Portions of the roof and combustible materials in the room burned until the fire department extinguished the fires. Eight people in the room were injured, one perished 15 days after the event due to thirddegree burns over $60 \%$ of his body and having suffered a ruptured liver from the event trauma. Of the seven other staff and researchers, one had severe burns, one had serious burns, one had burns and back injury, two had modest burns, one suffered head injuries and lacerations, and the last person had injuries to his chin and leg. Also, two firefighters were injured, one from smoke inhalation and one suffered a laceration that required stitches (WASH, 1975). This facility cost $\$ 11.6 \mathrm{M}$ to construct, and the fire damage amounted to $\$ 1.45 \mathrm{M}$ to repair the building and replace the equipment. These catastrophic explosions and fire resulted in a loss at over $12 \%$ of the initial cost of the facility (AEC, 1966). This event is definitely a case where a component failure produced catastrophic results.

Dewald (1988) discussed a fire that broke out in a duoplasmatron ion source at the Cologne tandem accelerator in the summer of 1984. The fire was most likely caused by a defective resistor or a high voltage discharge on top of the ion source chamber. The fire ignited under the plexiglass shielding. The shielding, cables, gas tubes, polyvinyl chloride tubes, and resistors were completely destroyed; plaster fell from the ceiling due to the intense heat and the smoke blackened the walls and ceiling. The burning polyvinyl chloride released $\mathrm{HCl}$ gas, which caused a great deal of corrosion to aluminum and 
stainless steel surfaces, power supply contacts, electrical wiring (insulation and wire), and other surfaces. The accelerator was down for over 6 months to clean the facility and repair the fire damage.

The following event has been included here due to the fact that the same type of operation occurs at accelerators and fusion experiments. The Los Alamos National Laboratory Gas Facility was receiving a gas shipment via a tube trailer on June 3, 1981. A hydrogen tube trailer and an oxygen tube trailer were simultaneously connected to the same manifold. There were insufficient barriers - only one shutoff valve - between the two types of gas. Incorrect purging procedures contributed to the event by allowing damage to the one valve and subsequent flow of higher pressure oxygen into one tube of the hydrogen trailer. Investigators believed that ignition was caused by contamination (i.e., sand particles) traveling through the valve at high velocity. The resulting explosion ruptured the tube, propelling tube fragments up to $380 \mathrm{~m}$ away from the trailer and creating a fireball of short duration. The fireball caused first and second degree burns over about $30 \%$ of the body of each of the two employees. One of the employees also suffered a small area of third degree burns. The tube trailer suffered major damage, the facility suffered minor damage. The total estimated damage was $\$ 25 \mathrm{k}$ (DOE, 1982). The accident investigation revealed that the cause was inadvertent mixing of hydrogen and oxygen due to insufficient technical and safety training of the personnel involved, lack of management control of the operation, lack of standard operating procedures for the task being attempted, and inadequate/poorly maintained equipment.

The CAIRS web site provided this information: the Superconducting Super Collider construction site near Waxahachie. Texas, suffered a construction accident. A construction worker was killed on January 29, 1993. An employee of the Obayashi/Dillingham company, a subcontractor at the SSC project site, was fatally injured when a concrete tunnel segment fell, crushing him against the segment erection device.

\subsection{Summary}

Figure 9 gives the DOE safety performance data for all research and development facilities from 1993-2003. Comparisons of these overall rate data to Figures 1, 3, 5, and 7 show that for staff members, PPPL varies between being equal to the yearly 'all DOE research' values and having much higher rate values, SLAC varies between being equal to the 'all DOE research' values and having about double the rate values, FNAL is about the same as the 'all DOE research' values, and CEBAF/Jlab is usually slightly below the 'all DOE research' values for lost work day rates. For services subcontractors, SLAC and FNAL were much higher than the 'all DOE research' values, CEBAF was lower, and PPPL did not list services separately. For construction, SLAC had rates generally higher by factors of 2 to 3, FNAL was slightly lower than the DOE values (except for a few peak years), and CEBAF was generally lower than the DOE rates except for one spike 


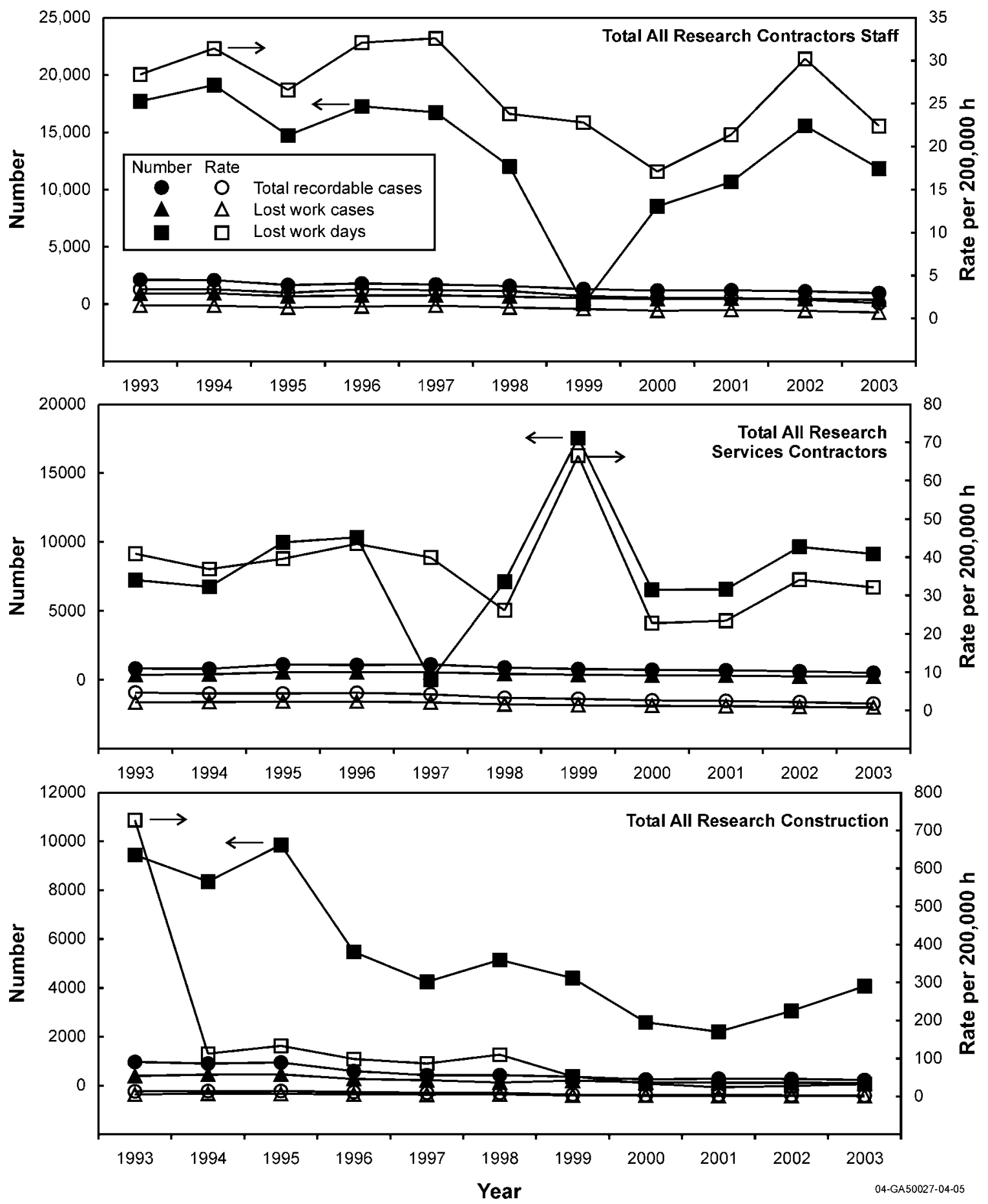

Figure 9. Plots of All DOE Research Industrial Safety Performance from 1993-2003. 
upward. Both SLAC and FNAL had construction fatalities, as did the Superconducting Super Collider; but CEBAF and RHIC did not experience any construction fatalities. These fatality results may be due to the time frames of the construction; SLAC and FNAL main construction was in the 1960's (before the comprehensive OSHA regulations were in effect) and the early 1970's (when OSHA was a new administration, having just come into effect in 1971). In the 1980's and 1990's, when OSHA regulations had become more enforced in the construction industry, the overall trend was decreased annual numbers of fatalities. The additional construction at FNAL and CEBAF in the 1990's and 2000's has been completed without any construction fatalities. The data show that there have not been any staff or subcontractor fatalities in the operation and maintenance of the accelerators, but there have been high consequence occupational accidents, usually dealing with electrical energy. Therefore, a strong program of electrical safety is considered essential for future experiments.

The initial expectation was that since FNAL is a worldwide leading particle accelerator and that SLAC is also a major facility in the world, that the facilities would be operated very professionally. However, the safety records show that these machines are not always operated with trend-setting safety or with best practices. Busick (1979) described that experimenters are human and possess human responses to pressure. As experimental apparatus becomes more complex and costly, time pressure increases. Safeguards are often viewed as serious impediments to experimental necessity. When pressures mount and safety barriers are in the way there is strong desire to bypass such barriers. Busick advocated enlightenment rather than enforcement as the means to obtain compliance with safety requirements; explaining the reasons for safety barriers rather than handing out punishment when a worker was caught in a violation. Busick believed that willing support of the experimenters and staff was essential to provide a safe working environment. Patterson (1994) describes the growth of high energy physics post-WW II. There was a somewhat cavalier attitude toward chronic radiation exposure, the only safety respect that was given by the research staff was for possible large, acute radiation doses. This was because there was adequate and accepted proof at the time that these doses could incur great harm or even be lethal. The experimenters were definitely in charge of the machines, it was nearly impossible for safety personnel to keep the experimenters and users from the machine even though close contact with the machine meant radiation exposure. Experimenters and technicians would walk and crawl over all parts of the machines; they only acknowledged dangers were present when the beam was on. If radiation safety was taken cavalierly, then we can be reasonably certain that industrial safety was not diligently addressed. This sort of attitude seems to have survived in the institutional memory of these facilities despite the DOE and OSHA rules and regulations that the facilities must comply with. Overall, FNAL and SLAC produce physics results and have accomplished these results without any operational fatalities. They have experienced occupational accidents, but these accident events have been accepted by the DOE. While these losses may be acceptable, it is nonetheless clear that safe operations are good business. In an example from another industry, Mottel (1995) 
described the DuPont company's safety story. Mottel pointed out that while safety programs cost money, the savings by safe operations are greater than the safety program costs. The DuPont company has operated safety programs that do not cost significantly more than other companies, but DuPont has had lost work case rates a factor of 10 less than the comparable chemical industry. The savings in medical costs and replacement work alone recoup the safety program costs. Mottel also describes some other savings: lost work time accidents generally mean replacing a skilled employee with a less skilled backup employee, safe performance minimizes the disruption of efficient plant operations, safe performance enhances maintenance at optimum operating conditions and minimizes damage to equipment and materials, and safe workers perform better than unsafe workers. Mottel also noted that employee morale is much higher in high safety facilities. Even when workers complain about safety requirements slowing their progress, they still tend to mentally equate the time, funding, and effort spent on safety as indications that management is interested in them as individuals. With such good morale built up, the total group works toward productive goals, including safe and efficient operations. Mottel also stated that at DuPont, employees and managers had clear safety goals, everyone understood that safety was a condition of continued employment. Since ITER is higher risk than present fusion experiments, continued attention to occupational safety is needed.

Another important issue seen from the ORPS reports and accident investigations is that more and more of the work has been subcontracted to outside companies as cost savings and time efficiency measures. Cost savings may have been realized by keeping smaller maintenance staff, but from the ORPS and accident reports it is also clear that there has been inadequate supervision by accelerator staff to determine that the subcontractors were meeting their safety obligations as specified in their contracts. This inadequate oversight combined with inadequate subcontractor safety and lack of subcontractor familiarity with the facilities has resulted in some of the highest consequence events at the accelerators, including electric arc burns, falls, chemical combustion accidents, and other events. If ITER hires subcontractors, then proper oversight of the subcontractors is necessary to enforce occupational safety. 


\section{Chapter 3 References}

AEC, 1966. Report on Investigation of Explosion and Fire Experimental Hall Cambridge Electron Accelerator, Cambridge, Massachusetts, July 5, 1965, TID-22594, United States Atomic Energy Commission, February 1966.

Bennett, 2000. D. B. Bennett, D. E. Paquette, K. Klaus, and W. R. Dorsch, Brookhaven National Laboratory Source Water Assessment for Drinking Water Supply Wells, BNL-52608, Brookhaven National Laboratory, 2000.

BNL, 1988. Brookhaven National Laboratory Annual Industrial Summary of Fire and Other Property Damage Experience, CY 1988, available from the BNL web site, bnl.gov/emergencyservices/.

Briggs, 2001. S. K. Briggs and S. V. Musolino, Preventing Pollution Using ISO 14001 at a Particle Accelerator, the Relativistic Heavy Ion Collider Project, BNL-68666, Brookhaven National Laboratory, 2001.

Busick, 1979. D. D. Busick and G. J. Warren, A Unique Safety Manual for Experimental Personnel, SLAC-Pub-2384, August 1979.

Cadwallader, 1991. L C. Cadwallader, Magnet Operating Experience Review for Fusion Applications, EGG-FSP-9977, Idaho National Engineering Laboratory, November 1991.

Cadwallader, 1997. L. C. Cadwallader, Lift Truck Safety Review, INEL/EXT-97-00178, Idaho National Engineering Laboratory, March 1997.

Cadwallader, 2003. L. C. Cadwallader and P. I. Petersen, "Reliability Estimates for Oxygen Monitors," Proceedings of the $20^{\text {th }}$ IEEE/NPSS Symposium on Fusion Engineering, San Diego, California, October 14-17, 2003, IEEE, 2003, pages 171174.

Chao, 1999. A. W. Chao and M. Tigner, Handbook of Accelerator Physics and Engineering, World Scientific Publishing Company, Singapore, 1999, section 8.

Cossairt, 2000. J. D. Cossairt, Environment, Safety, and Health Considerations for a Neutrino Source Based on a Muon Storage Ring, FERMILAB-TM-2111, Fermi National Accelerator Laboratory, May 2000.

Dewald, 1988. A. Dewald and L. Steinert, "Reconditioning of the Cologne Tandem after a Fire at the Ion Source," Nuclear Instruments and Methods in Physics Research A, 268 (1988) 356-360. 
DOE, 1980. Operational Accidents and Radiation Exposures at ERDA Facilities 19751977, DOE/EV-0080, US Department of Energy, 1980.

DOE, 1980a. Operational Accidents and Radiation Exposures at DOE Facilities, Fiscal Year 1978, DOE/EV-0091/1, US Department of Energy, 1980.

DOE, 1982. Environment, Safety and Health at DOE Facilities, Annual Report Calendar Year 1981, DOE/EP-0024/1, US Department of Energy, October 1982.

DOE, 1984. Environment, Safety and Health at DOE Facilities, Annual Report Calendar Year 1982, DOE/PE-0055, US Department of Energy, 1984.

DOE, 1987. Investigation Report of the Fire in the Wide Band Laboratory at Fermi National Accelerator Laboratory Batavia, Illinois October 3, 1987, cataloged as DOE/MISC-108 at the INEEL, US Department of Energy, 1987.

DOE, 1991. Tiger Team Assessment of the Stanford Linear Accelerator Center, DOE/EH-0243, US Department of Energy, November 1991.

DOE, 1992. Tiger Team Assessment of the Fermi National Accelerator Laboratory, DOE/EH-0250, US Department of Energy, June 1992.

DOE, 1992a. Tiger Team Assessments Seventeen through Thirty-Five: A Summary and Analysis, DOE/EH-0302-V2, US Department of Energy, December 1992, ch. 4.

DOE, 1997. Type B Accident Investigation Board Report on the October 22, 1997, Electrical Arc Blast at Building F-Zero at Fermi National Accelerator Laboratory, Batavia, Illinois, US Department of Energy, Chicago Operations Office, November 1997.

DOE, 1998. Type B Accident Investigation Board Report on the September 4, 1998, Flammable Liquid Fire/Explosion at Fermi National Accelerator Laboratory, Batavia, Illinois, US Department of Energy, Chicago Operations Office, October 16, 1998.

DOE, 2001. Type A Accident Investigation Report of the June 21, 2001 Drilling Rig Operator Injury at the Fermi National Accelerator Laboratory, US DOE Office of Environment, Safety and Health Oversight, August 2001.

DOE, 2003. Type B Accident Investigation of the January 28, 2003 Fall and Injury at the Stanford Linear Accelerator Center, US Department of Energy, Stanford Site Office, February 2003. 
DOE, 2004. Type A Accident Investigation of the Electrical Arc Injury on October 11, 2004, at the Stanford Linear Accelerator Center, Menlo Park, California, US DOE, November 2004.

Ingrassia, 2001. "Safety Issues in Accelerator Operation: Groundwater Contamination," Workshop on Accelerator Operation, WAO 2001, Villars sur-Ollon, Switzerland, 28 January - 2 February, 2001, pages 128-130. Available at CERN document server/library web site.

Johnson, 1987. R. Johnson, "Initial Operation of the Tevatron Collider," Proceedings of the Particle Accelerator Conference, Washington, DC, March 16-19, 1987, IEEE, 1987, pages 8-12.

Lyon, 1991. R. E. Lyon, Safety Performance Profile, Stanford Linear Accelerator Center, EGG-SSDC-9913, Idaho National Engineering Laboratory, September 1991.

Mau, 2001. B. Mau, "Tools to Control Large Superconducting Colliders," Workshop on Accelerator Operation, WAO 2001, Villars sur-Ollon, Switzerland, 28 January - 2 February, 2001, pages 159-162. Available at CERN document server/library web site.

Mottel, 1995. W. J. Mottel, J. F. Long, and D. E. Morrison, Industrial Safety is Good Business, the DuPont Story, John Wiley \& Sons, Inc., New York, 1995, chapters 2,7 .

Neal, 1965. R. B. Neal, "The Stanford Two-Mile Linear Electron Accelerator," Journal of Vacuum Science and Technology, 2 (1965) 149-159.

Patterson, 1994. H. W. Patterson and R. H. Thomas, editors, The History of Accelerator Radiological Protection, Personal and Professional Memoirs, Nuclear Technology Publishing, Kent, England, 1994.

Trbojevic, 2001. D. Trbojevic et al., "Commissioning of the Relativistic Heavy Ion Collider," Proceedings of the 2001 Particle Accelerator Conference, Chicago, Illinois, June 18-22, 2001, IEEE, 2001, pages 3138-3140.

WASH, 1975. Operational Accidents and Radiation Exposure Experience within the United States Atomic Energy Commission, 1943-1975, WASH-1192, US Atomic Energy Commission, Fall 1975. 
Zinkann, 2001. G. P. Zinkann, "Operating ATLAS; The World's First Supercondicting Heavy-Ion Accelerator," Workshop on Accelerator Operation, WAO 2001, Villars sur-Ollon, Switzerland, 28 January - 2 February, 2001, pages 183-187. Available at CERN document server/library web site. 


\subsection{NUCLEAR POWER PLANT OCCUPATIONAL SAFETY}

This chapter presents data gathered from the US nuclear industry about the light water reactor nuclear power plants, to allow general comparisons to the other data from DOE experiment facilities. Power plant operation is covered by the US Bureau of Labor Statistics (BLS) under 'electric services', but this category includes all types of power plants. These include coal-fired plants, oil and gas burning plants, hydroelectric dams, wind farms and solar plants, pumped storage, compressed air storage, and nuclear plants. The data have been binned too broadly for close comparisons. Yager (2001) noted this, and the author verified this same observation with the BLS. Nuclear plants are about $20 \%$ of the US electrical power production; however, nuclear power plants tend to employ more staff than other types of power plants so using a scaling factor of $20 \%$ of the electric services category values would be inaccurate. Also, scanning news reports indicates that there are accidents involved with the material handling of large quantities of coal, including rail accidents, injuries from moving coal at the plant site and crushing the coal before burning, and the occasional explosion of coal dust. There have also been several events of steam line breaks in coal-fired plants that resulted in fatalities, the worst event in the US being the Mohave power plant event in June 1985, which resulted in six employee fatalities and ten employees being seriously injured (Bangs, 1986).

The reader should recall that the first commercial nuclear power plant in the US was the Shippingport pressurized water reactor (PWR) that came on line in 1957, and the next plants were the Dresden I boiling water reactor (BWR) and the Yankee Rowe PWR in 1960, and the Big Rock Point BWR in 1963. There were 18 plants on line by mid-1965. After that, dozens more plants came on line in the rest of the 1960's and the 1970's. After the Three Mile Island accident in 1979, new plant orders were retracted, and only a few plants that were under construction have been completed since then. There are now just over 100 operating power plants in the US. As stated above, the BLS does not partition its data by the type of plant that an electric power industry worker chooses as his/her vocation. Therefore, other data sets have been sought. The earliest occupational safety data located was a series of industry-specific reports that the US Atomic Energy Commission requested from the BLS. These data spanned 1965-1970 (BLS, 1967; BLS, 1969; BLS, 1970; BLS 1971; BLS, 1972) and are presented in Table 4. There seems to be great reluctance on the part of the nuclear power plant companies to discuss their occupational injuries or fatalities. Perhaps this is due to the nuclear industry's concern that their industrial injuries and fatalities would be misconstrued by the media and the public as being related to ionizing radiation when they are not.

Other sources of occupational safety data have also been compiled and published. O'Donnell (1982) referred to the data in Table 4, and they used plant data from the Tennessee Valley Authority, that was operating several nuclear power plants at that time (Browns Ferry 1, 2, and 3 were all operational by 1977; the Sequoyah and Watt's Bar plants came on line later). This is only a small sample of the 100 US power plants that now operate. 
Table 4. Early Values of Occupational Safety Rates for the Nuclear Fission Industry

\begin{tabular}{|c|c|c|c|c|c|c|}
\hline \multirow[b]{2}{*}{ Type of Activity } & \multicolumn{6}{|c|}{ Annual Injury Frequency Rate } \\
\hline & 1965 & 1966 & 1967 & 1968 & 1969 & 1970 \\
\hline $\begin{array}{l}\text { Production of special } \\
\text { materials for use in } \\
\text { reactors } \\
\text { (graphite, beryllium, } \\
\text { etc.) }\end{array}$ & $\begin{array}{c}9.2 \\
(0.018)\end{array}$ & $\begin{array}{c}13.3 \\
(0.027)\end{array}$ & $\begin{array}{c}25.2 \\
(0.050)\end{array}$ & $\begin{array}{c}36.8 \\
(0.074)\end{array}$ & $\begin{array}{c}28.2 \\
(0.056)\end{array}$ & $\begin{array}{c}42.6 \\
(0.085)\end{array}$ \\
\hline $\begin{array}{l}\text { Fuel element } \\
\text { fabrication }\end{array}$ & $\begin{array}{c}4.2 \\
(0.008)\end{array}$ & $\begin{array}{c}4.6 \\
(0.009)\end{array}$ & $\begin{array}{c}6.9 \\
(0.014)\end{array}$ & $\begin{array}{c}5.3 \\
(0.011)\end{array}$ & $\begin{array}{c}7.1 \\
(0.014)\end{array}$ & $\begin{array}{c}6.4 \\
(0.013)\end{array}$ \\
\hline $\begin{array}{l}\text { Reactor and reactor } \\
\text { component design and } \\
\text { manufacture }\end{array}$ & $\begin{array}{c}4.2 \\
(0.008)\end{array}$ & $\begin{array}{c}4.0 \\
(0.008)\end{array}$ & $\begin{array}{c}2.8 \\
(0.006)\end{array}$ & $\begin{array}{c}4.8 \\
(0.01)\end{array}$ & $\begin{array}{c}4.7 \\
(0.01)\end{array}$ & $\begin{array}{c}3.3 \\
(0.007)\end{array}$ \\
\hline $\begin{array}{l}\text { Design and engineering } \\
\text { of nuclear facilities }\end{array}$ & $\begin{array}{c}7.2 \\
(0.014) \\
\end{array}$ & $\begin{array}{c}5.9 \\
(0.012) \\
\end{array}$ & $\begin{array}{c}10.5 \\
(0.021)\end{array}$ & $\begin{array}{c}1.4 \\
(0.003)\end{array}$ & $\begin{array}{c}1.6 \\
(0.003)\end{array}$ & $\begin{array}{c}2.3 \\
(0.005) \\
\end{array}$ \\
\hline $\begin{array}{l}\text { Power reactor operation } \\
\text { and maintenance }\end{array}$ & $\begin{array}{c}3.3 \\
(0.007)\end{array}$ & $\begin{array}{c}3.9 \\
(0.008)\end{array}$ & $\begin{array}{c}5.0 \\
(0.01)\end{array}$ & $\mathrm{n} / \mathrm{c}$ & $\mathrm{n} / \mathrm{c}$ & $\begin{array}{c}7.9 \\
(0.016)\end{array}$ \\
\hline $\begin{array}{l}\text { Nuclear instruments } \\
\text { manufacturing }\end{array}$ & $\begin{array}{c}3.8 \\
(0.008) \\
\end{array}$ & $\begin{array}{c}5.8 \\
(0.012) \\
\end{array}$ & $\begin{array}{c}5.5 \\
(0.011) \\
\end{array}$ & $\begin{array}{c}6.8 \\
(0.014)\end{array}$ & $\begin{array}{c}6.2 \\
(0.012) \\
\end{array}$ & $\begin{array}{c}7.9 \\
(0.016) \\
\end{array}$ \\
\hline $\begin{array}{l}\text { Processing and } \\
\text { packaging } \\
\text { radioisotopes }\end{array}$ & $\begin{array}{c}1.3 \\
(0.003)\end{array}$ & $\begin{array}{c}1.8 \\
(0.004)\end{array}$ & $\begin{array}{c}7.9 \\
(0.016)\end{array}$ & $\begin{array}{c}6.5 \\
(0.013)\end{array}$ & $\mathrm{n} / \mathrm{c}$ & $\begin{array}{c}4.5 \\
(0.009)\end{array}$ \\
\hline Private research labs & $\begin{array}{c}3.7 \\
(0.007)\end{array}$ & $\begin{array}{c}4.7 \\
(0.009)\end{array}$ & $\begin{array}{c}2.7 \\
(0.005)\end{array}$ & $\begin{array}{c}4.0 \\
(0.008)\end{array}$ & $\begin{array}{c}4.6 \\
(0.009)\end{array}$ & $\begin{array}{c}4.0 \\
(0.008)\end{array}$ \\
\hline $\begin{array}{l}\text { Injury distribution (\%) } \\
\text { death } \\
\text { permanent impairments } \\
\text { temporary impairments }\end{array}$ & $\begin{array}{r}1.1 \\
5.8 \\
93.1\end{array}$ & $\begin{array}{r}1.3 \\
4.7 \\
94.0\end{array}$ & $\begin{array}{r}0.5 \\
2.9 \\
96.6\end{array}$ & $\begin{array}{c}1.0 \\
3.3 \\
95.7\end{array}$ & $\begin{array}{c}0.7 \\
3.8 \\
95.5\end{array}$ & $\begin{array}{l}0.8 \\
2.6 \\
96.6\end{array}$ \\
\hline $\begin{array}{l}\text { Notes: The injury freque } \\
\text { per year. Assuming a st } \\
\text { per year. The second nu } \\
\text { "n/c" stands for not cited } \\
\text { reliability (e.g., low valu }\end{array}$ & dat & om & $\begin{array}{l}\text { inju } \\
\text { hou } \\
\text { e ant } \\
\text { they }\end{array}$ & $\begin{array}{l}\text { rate } \\
\text { te pe } \\
\text { of qu } \\
\text { ed to }\end{array}$ & $\begin{array}{l}\text { mpl } \\
\text { r } 50 \\
\text { vidu } \\
\text { nabl } \\
\text {. }\end{array}$ & $\begin{array}{l}\text { lours } \\
\text { kers } \\
\text { ker. } \\
\text { stical }\end{array}$ \\
\hline
\end{tabular}


O’Donnell data from Tennessee Valley Authority plants is given below:

$\begin{array}{lllll}\underline{\text { Incidence Rates }} & \underline{1975} & \underline{1976} & \underline{1977} & \\ \begin{array}{l}\text { fatalities } \\ \text { per 100 man-years }\end{array} & 0.0 & 0.0 & 0.0 & \text { (note: they assumed 0.01/100 man-years) } \\ \begin{array}{l}\text { Serious injuries } \\ \text { per 100 man-years }\end{array} & 0.38 & 0.27 & 1.7 & \end{array}$

Cochran (1999) reported some data from a Nuclear News magazine article (Rippon, 1981) that outlined worker injury rates that had been complied for a United Kingdom safety report; this report also cited other data sources. Operating a nuclear power station of $1,000 \mathrm{MWe}$ at $75 \%$ availability gave these results:

\begin{tabular}{lll} 
& \multicolumn{2}{c}{ Nuclear power plant annual operation (including reprocessing) } \\
\cline { 2 - 3 } Study & Accidental injuries per 750 MWe & Accidental deaths per 750 MWe \\
\cline { 2 - 3 } Inhaber & 1.37 & $0.01-0.013$ \\
Norwegian & $0.7-2.8$ & $0.01-0.2$ \\
Swedish & 11.1 & 0.03 \\
WASH-1224 & 1.42 & 0.012 \\
Comar \& Sagan & 1.3 & 0.01 \\
Fagnani \& Maccia & 4.9 & 0.013 \\
Hamilton & 1.3 & 0.015 \\
Black \& Niehaus & 3.48 & 0.011 (this value excludes reprocessing) \\
& & \\
Ranges & $0.7-11.1$ & $0.01-0.2$
\end{tabular}

To convert the UK report values to injuries and fatalities per worker-year, the average number of workers at a nuclear power plant is needed. Not all the sources above were US data, and only counts of US workers at nuclear plants are available, but these are probably similar to UK staffing counts. Due to the assumption of similar staffing, the conversion will only be applied to the ranges of values. The annual radiation exposure NUREG (Burrows, 2002) gives the number of workers monitored for radiation exposure in all of the 104 commercial power plants in the US. This number of monitored workers was 140,776 for 2001 and the average number of workers per plant is rounded off to 1,350. The Statistical Abstract of the US for 2002 cited 354,000 workers in the overall electrical services industry (code 491). Therefore, nuclear plants that produce about $20 \%$ of the nation's power employ $40 \%$ of the electric services workers, meaning that nuclear plants are more highly staffed than coal-fired, hydroelectric, and other types of power plants. These data qualitatively agree with industry claims of high amounts of staffing at nuclear versus other power facilities. As a first approximation, we assume that most of the US plants are large output; this is reasonable since the first several plants that were small power output have been decommissioned (e.g., Shippingport, Dresden 1, Big Rock 
Point, Yankee Rowe). Using this average number of workers per plant value to match the 1,000 MWe plant, a new range values has been calculated for the data given above:

\section{$\underline{\text { Accidental injuries per worker-year }}$ Accidental deaths per worker-year}
Adjusted Ranges
5.19E-04 - 8.22E-03
7.4E-06 - 1.48E-03

The US industry has had a more recent publication of occupational safety data in Nuclear News (NN, 2001). These data were compiled by the US Institute of Nuclear Power Operations (INPO). Their compilation was based on 200,000 worker-hours, with a standard work year being 2,000 hours. Yearly aggregate values for lost work time injuries, injuries resulting in restricted work, and fatalities (combined under the term "accidents") for nuclear power plants have been given as:

\begin{tabular}{lcl} 
Calendar year & $\begin{array}{l}\text { Accidents per } \\
\text { 200,000 worker-hours }\end{array}$ & $\begin{array}{l}\text { Accidents per } \\
\text { worker-year }\end{array}$ \\
\cline { 2 - 2 } 1980 & 2.10 & 0.0210 \\
1984 & 1.50 & 0.0150 \\
1988 & 1.34 & 0.0134 \\
1990 & 1.03 & 0.0103 \\
1992 & 0.77 & 0.0077 \\
1994 & 0.64 & 0.0064 \\
1996 & 0.46 & 0.0046 \\
1998 & 0.29 & 0.0029 \\
1999 & 0.34 & 0.0034 \\
2000 & 0.26 & 0.0026
\end{tabular}

The stated 1998-1999-2000 goal value was 0.40 accidents/200,000 worker-hours, or 0.004 accidents per worker-year. Note the factor of 8 decrease in the accident rate from 1980 to 2000, even though the number of plants coming on line was still slowly increasing throughout the 1980's and the plants were increasing their on-line time from $\sim 70 \%$ availability to $\sim 90 \%$ availability. INPO has also published a performance indicators update (INPO, 2003) that gives 2001 and 2002 values of 0.24 and 0.22 accidents per 200,000 worker-hours. Loomis (1999) stated that in a study of five power companies (operating mainly fossil fueled plants) that included 1,454,370 worker-years, the fatality rate was $0.0132 /$ worker-year. The Loomis study listed causes of death, in descending order, as electric current exposure, homicide, falls from height, exposure to explosions, 'struck by object', drowning, burns, machinery-related, and motor vehicle accidents. The electrical events were $45 \%$ of the deaths, and were divided into high energy work (i.e., linemen, substation operators, and cable splicers), medium energy work (i.e., mechanics, machinists, operators, welders who work near energized buses and switchgear), and low energy work, such as that performed by instrumentation and controls technicians, relay technicians, telecommunication technicians, and riggers.

The World Association of Nuclear Operators (WANO) has also published some occupational safety data that has been shared from member utilities (WANO, 2003). The 
accident rate is for industrial accidents that result in lost work time, restricted work, or fatalities. These data are summarized below for comparison to the Nuclear News/INPO data:

$\begin{array}{lccc}\begin{array}{l}\text { Calendar } \\ \text { Year }\end{array} & \begin{array}{c}\text { Number of } \\ \text { Power Plants Reporting }\end{array} & \begin{array}{c}\text { Accident Rate per } \\ \text { 200,000 man-hours }\end{array} & \begin{array}{l}\text { Accident Rate } \\ \text { per worker-yr }\end{array} \\ 1990 & 169 & 1.04 & 0.0104 \\ 1992 & 175 & 0.80 & 0.0080 \\ 1994 & 183 & 0.63 & 0.0063 \\ 1996 & 198 & 0.51 & 0.0051 \\ 1998 & 202 & 0.42 & 0.0042 \\ 2000 & 203 & 0.33 & 0.0033 \\ 2001 & 200 & 0.33 & 0.0033 \\ 2002 & 203 & 0.31 & 0.0031 \\ 2003 & 201 & 0.28 & 0.0028\end{array}$

The US is a member of WANO, and the count of US nuclear power plants in the 19902003 time span was about 100; consequently there are data from about 100 other plants in other WANO member countries represented in this table. The numbers closely agree to the Nuclear News/INPO values. Therefore, of the 400 worldwide reactors, the occupational safety rates at half the plants are quite favorable.

These statistical data were the only published data found in an extensive literature search. Assuming that these data are essentially correct, there remains the issue of how to decompose the reported "accident" data into fatality and injury events. The earliest data compiled by the BLS held some percentage values that have been given at the bottom of Table 4. It is reasonable to assume that two factors would tend to reduce the cited percentages of death in occupational accidents. The first is the OSHA regulations that came into effect in 1971. While the set of BLS reports shows that the nuclear industry was typically experiencing fewer incidents than general industry, some aspects of the OSHA regulations would still impact power plant safety; for example, electrical safety and ladder safety regulations. The OSHA regulations have had the overall industry effect of reducing occupational fatalities throughout the country. From the National Institute of Occupational Safety and Health web site cdc.gov/niosh:

"The numbers and rates of traumatic occupational fatalities declined from 1980 through 1998. The number of fatal occupational injuries decreased $35 \%$, from 7,343 fatalities in 1980 to 4,798 in 1998 . The annual fatality rate per 100,000 civilian workers decreased $47 \%$, from 7.4 in 1980 to 3.9 in 1998."

The second factor responsible for saving lives is the increasing medical knowledge and increased levels of emergency medical care, with paramedics and emergency medical technicians that can administer first aid and lifesaving measures much more quickly than the 1950's and 1960's ambulances that merely transported an injured person to a hospital. The exact count of recent annual fatalities at nuclear power plants is not readily available, 
but fatality counts are believed to be only a few persons each year, from causes such as electrocutions, vehicle accidents, falls and other events. Considering 2002, when the only known work-related fatality at a US nuclear plant was a worker who was electrocuted while trimming tree branches near a $138 \mathrm{kV}$ line on a reactor site (LER, 2002), then the fatal accident ratio is $1 / 140,776$ or $7.1 \mathrm{E}-06$ fatalities per worker-year. Combining with 0.0022 accidents per worker-year for 2002 from INPO, the fatality percentage is $0.32 \%$. Comparing to the data in Table $4-1$, this is a small decrease in the fatality percentage, but consideration must also be given to the fact that the industry has also expanded a great deal in $\sim 30$ years.

An overall table of the commercial power plant operation occupational safety data is given below, on a per plant worker basis. Subcontractors are not included in these estimates:

$\begin{array}{lllll}\underline{\text { Year }} & \begin{array}{l}\text { Accident rate } \\ \text { per worker-year }\end{array} & \begin{array}{l}\text { Fatality rate } \\ \text { per worker-year }\end{array} & \text { Source } \\ 1965 & 0.007 & 7.7 \mathrm{E}-05 & \\ 1966 & 0.008 & 1.0 \mathrm{E}-04 & \text { (BLS, 1967) } \\ 1967 & 0.01 & 5.0 \mathrm{E}-05 & \text { (BLS, 1967) } \\ 1968 & \mathrm{n} / \mathrm{c} & \mathrm{n} / \mathrm{c} & \text { (BLS, 1969) } \\ 1969 & \mathrm{n} / \mathrm{c} & \mathrm{n} / \mathrm{c} & \text { (BLS, 1970) } \\ 1970 & 0.016 & 1.3 \mathrm{E}-04 & \text { (BLS, 1971) } & \text { (BLS, 1972) }\end{array}$

Data from only two plants:

$\begin{array}{llll}1975 & 0.0038 & 1 \mathrm{E}-04 \text { (assumed) } & \text { (O’Donnell, 1982) } \\ 1976 & 0.0027 & 1 \mathrm{E}-04 \text { (assumed) } & \text { (O'Donnell, 1982) } \\ 1977 & 0.017 & 1 \mathrm{E}-04 \text { (assumed) } & \text { (O'Donnell, 1982) }\end{array}$

$\begin{array}{llll}1980 & 0.0210 & 1.3 \mathrm{E}-04 & \text { (NN, 2001), (assumed 0.6\% deaths) } \\ 1984 & 0.0150 & 9.0 \mathrm{E}-05 & \text { (NN, 2001), (assumed 0.6\% deaths) } \\ 1985 & 0.0110 & 6.6 \mathrm{E}-05 & \text { (INPO, 2003), (assumed 0.6\% deaths) } \\ 1988 & 0.0134 & 8.0 \mathrm{E}-05 & \text { (NN, 2001), (assumed 0.6\% deaths) } \\ 1990 & 0.0103 & 4.6 \mathrm{E}-05 & \text { (NN, 2001), (assumed 0.6\% deaths) } \\ 1992 & 0.0077 & 3.5 \mathrm{E}-05 & \text { (NN, 2001), (assumed 0.45\% deaths) } \\ 1994 & 0.0064 & 2.9 \mathrm{E}-05 & \text { (NN, 2001), (assumed 0.45\% deaths) } \\ 1995 & 0.0055 & 2.5 \mathrm{E}-05 & \text { (INPO, 2003), (assumed } 0.45 \% \text { deaths) } \\ 1996 & 0.0046 & 2.1 \mathrm{E}-05 & \text { (NN, 2001), (assumed 0.45\% deaths) } \\ 1997 & 0.0045 & 2.0 \mathrm{E}-05 & \text { (INPO, 2003), (assumed 0.45\% deaths) } \\ 1998 & 0.0029 & 1.3 \mathrm{E}-05 & \text { (NN, 2001), (assumed 0.45\% deaths) } \\ 1999 & 0.0034 & 1.5 \mathrm{E}-05 & \text { (NN, 2001), (assumed 0.45\% deaths) } \\ 2000 & 0.0026 & 8.3 \mathrm{E}-06 & \text { (NN, 2001), used 0.32\% deaths } \\ 2001 & 0.0024 & 7.7 \mathrm{E}-06 & \text { (INPO, 2003), used 0.32\% deaths } \\ 2002 & 0.0022 & 7.0 \mathrm{E}-06 & \text { (INPO, 2003), used 0.32\% deaths }\end{array}$

The comparison values show that the accident rates at nuclear power plants have been decreasing with time, which means that fission plants have become safer places to work. 
The fission power industry is maturing; plant operators and maintainers, and their managers, have recognized the hazards and they mitigate or avoid hazards. US fission plants now operate in the $85-90 \%$ availability range per calendar year (Cadwallader, 1999), due to several factors - understanding the plant, the decrease in 'infant mortality' equipment failures that typically occur in the first few years of new plant operation, economics drivers, and the Nuclear Regulatory Commission's scram reduction program. What is notable for fission plants is that the most hazardous plant areas are no longer routinely occupied.

Unfortunately, the fission plant occupational safety data resolution does not allow estimation of the accident or fatality risk for individual occupations (e.g., welder, mechanic, electrician, etc.) or operation in the plant, such as maintenance, surveillance, or inspection tasks, operations evolutions, or other tasks. Only the yearly totals have been reported by INPO and WANO. These data apply only to the utility personnel, that is, the people who are permanently assigned to the power plant, not subcontractors who are hired for special tasks. Subcontractors were not included in these estimates. The data can only serve as bounding values for overall yearly safety estimates for fusion workers. From previous chapters, there have not been any occupational fatalities in operation of the fusion experiments or accelerators, so there is no fatality rate. Lost work case rates for PPPL, SLAC, FNAL and CEBAF, from Figures 1, 3, 5, and 7, have been compared to the accident rates given for fission plants and were very comparable in past decades, but now the fusion and accelerator rates are in the 0.02/worker-year range and the fission plants have reduced their accident rate to the 0.002/worker-year range. However, the severity of the accidents is usually quite low for the technology facilities (as seen in Figures 2, 4, 6, and 8) while the accident severity for the power plants is not known.

Scanning the NRC database and web site has given a few recent power plant events of industrial safety interest; however, these databases are not obligated to report occupational injuries that did not endanger the public with exposure to radiation. In 10CFR50.72, the NRC must be notified in case an event or situation at a licensee was related to the health or safety of the plant workers and the licensee planned to make a press release; such as an on-site fatality or the release of radioactive materials. This includes transporting a potentially contaminated worker off-site for medical treatment. Some injuries have also been reported as a courtesy to the NRC. Of course, occupational accidents and fatalities are reported to OSHA, but OSHA and the BLS do not publish data strictly for nuclear power plants. Some events of industrial safety concern from nuclear power plants are discussed below.

A few NRC publications have discussed industrial safety. Bertini (1980) and Sailor (1985) discussed several events: seven employees injured at the Robinson-2 PWR in 1970 when a safety valve failed; an employee was seriously injured when he was drawn through a manhole in a containment air lock at the Surry-2 PWR in 1973; two employees were injured in a hydrogen gas explosion from the off-gas system at the Cooper BWR in 1975; and one employee injured in a hydrogen gas explosion from the off-gas system at the Millstone-1 PWR in 1977. The workers at Robinson-2 were testing the secondary side safety relief valves before the plant was operational. When they began to attach the 
testing equipment to the ninth valve, a fan of steam jetted out and the valve catastrophically failed, tearing loose and shooting up about $40 \mathrm{~m}$. The workers were showered with debris and were being impinged by steam. They were either blown from the scaffolding to the floor or leapt down to avoid the steam. The seven men were transported by ambulance to the hospital, where they were treated for steam burns and injuries. Bertini also described the Surry-2 event with the worker drawn through the air lock on December 10, 1973. The plant was operating and the control room received an alarm that containment pressure was slightly increasing. An operator was sent to investigate the seals of the air lock doors. He entered the air lock but forgot to close the outer door and reduce the pressure in the passageway. As he was checking the seal to a 0.46-m diameter escape hatch, the hatch seal failed and the hatch opened. The air pressure difference between the containment and the passageway propelled the worker into the hatch and he fell $\sim 5 \mathrm{~m}$ into the containment building where he struck a crane. Other workers did not know what had happened to the employee; the plant shut down on the containment air pressure increasing and two more operators were sent to investigate. They saw the outer airlock door open and the inner hatch open; they assumed the first operator had entered the containment and that he was careless to leave both sides of the airlock open. They closed the outer door to re-establish containment pressure. After about 30 minutes, the injured man crawled back into the airlock but could not open it, and now the air pressure on the door was about $27 \mathrm{kPa}$ (4 psi), so the door could not be opened. The other workers found him in the airlock, and the containment had to be brought up to a higher pressure to be able to open the outer door and render aid to the operator. This took another 30 minutes. He was seriously injured, but he recovered from his injuries and returned to work.

Sailor (1985) and Bertini (1980) described explosions at the Millstone-1 PWR on December 13, 1977. During plant operation, a small hydrogen gas explosion occurred in the auxiliary building. The damage was minor; the cause was not certain. The plant continued to operate. Some of the water seals in the off-gas system were blown out in the first explosion. The seals were not properly refilled, so hydrogen leaked out of the offgas system (as it is designed to do). When enough hydrogen had accumulated in the auxiliary building, electrical equipment sparks ignited the gas and a larger explosion occurred about 3.5 hours later. The door to the auxiliary building was blown from its hinges and traveled $54.8 \mathrm{~m}$ (180 feet) where it struck the warehouse building. One man was seriously injured and was hospitalized for 4 days.

Bertini (1980) also described an event at the Surry-1 plant on July 27, 1972. Workers were attempting to vent steam from the secondary coolant system while the plant was in hot-standby to perform maintenance on the main turbine bypass valves. They manipulated the atmospheric steam dump valves, but these valves did not work. They tried another valve and one of the three men went outside to see if the steam was venting from the release line. It was not, and when he returned to the building he saw that the upper level where the valves were was full of steam. The other two men had become engulfed in steam. The third man called for valve closure and for help, but the two men were badly scalded and died four days later. In that plant design, the release line was not welded to the vent valve exhaust (presumably due to thermal expansion and contraction 
issues), it was sleeve fitted. On the day of the event, investigators believed that the valve hung up on the sleeve and then pushed downward farther than normal, so that steam escaped from the sleeve connection into the surrounding area.

On November 5, 1975, the Cooper power plant suffered a hydrogen gas explosion in the auxiliary building. A misaligned valve (that gave proper valve lineup indication in the control room) on the main condenser allowed steam, hydrogen and oxygen to back up into a sump in the auxiliary building. When the sump began to register high pressure, employees were sent to investigate. As one raised the manhole cover, the other turned on the air sampler (this is proper procedure for testing the atmosphere in the sump before entry). The air sampler apparently sparked, and the gas deflagrated. The one worker was seriously injured and the other received minor burns. They both recovered and returned to work.

On June 28, 1982, a feedwater heat extraction line failed at the Oconee 2 PWR (NRC, 1982). Two persons nearby suffered steam burns and had to be hospitalized. On December 9, 1986, a main feedwater pipe failed catastrophically at the Surry 2 PWR (NRC, 1986). Eight workers were nearby, replacing thermal insulation on other piping. Four of the workers died from the burns they received from flashing feedwater, two healed in the hospital, and two were treated and released from the hospital the day of the event. That event motivated the NRC to cause the utilities to make studies of high energy line breaks and map the hazardous areas in their plants.

The NRC has also published a notice (NRC, 1988) that described four fatal electrocution accidents among electricians at US nuclear power plants in the 1970's and 1980's. In 1971 at the Quad Cities BWR, an electrician was pulling cable and the cable inadvertently became energized, electrocuting him. In 1980, an electrical worker was cleaning breaker cubicles at the San Onofre PWR; he contacted a live $4 \mathrm{kV}$ line and was electrocuted (note that this event is similar, in type but not in outcome, to one of the FNAL events cited in chapter 3). In 1987 at the Wolf Creek PWR, and electrical technician was cleaning transformer cubicles and came in contact with an energized $4 \mathrm{kV}$ line, electrocuting him. In 1988, at the same plant, an electrician was attempting to pull additional wires through a conduit while the existing $480 \mathrm{~V}$ lines in the conduit were energized. During his work to get the new wires through the conduit, damaged insulation on the existing lines allowed a short circuit that electrocuted the electrician. On June 9, 1993, a quarterly in-service test of the high pressure coolant injection turbines was in progress at the Quad Cities plant. The turbine's exhaust steam line rupture disks burst during the test due to a pressure pulsation, and the five people in the room were burned by steam and were also slightly contaminated. The four workers performing the test were not severely injured, and, even though they were stated to have become complacent with the test procedure, they were not standing near the rupture disks. The fifth person, a health physics technician, was the most severely injured. The technician was making routine rounds and was not aware of the danger posed by the quarterly test (NRC, 1993). The steam release caused the room's fire doors to be blown off their hinges [they were probably rated for only $6.8 \mathrm{kPa}(1 \mathrm{psig})$ overpressure; the room was likely rated for high 
pressure but the doors were not]. The NRC notice stated that if the release had been from the turbine steam inlet rather than the exhaust, the damage would have been more severe.

A more recent event involved a contract worker trimming tree limbs and shrubbery behind the emergency diesel generator building, near a $138 \mathrm{kV}$ line, at the Indian Point 2 plant on July 19, 2002 (LER, 2002). The trimming work resulted in an electrical ground path through the worker, resulting in electrocution. A loss of offsite power event occurred at the power plant because of that short circuit to ground. Emergency medical personnel were not able to revive the worker.

Some other nuclear power plant worker injury events and near miss events were listed in the NRC Event Notification Reports and are summarized in Table 5. These events are of interest since they originate from failures of power plant environment, and many of the same types of equipment and systems could be used in ITER or other large fusion experiments. A few of these events are due to personnel errors (choosing their footing, using PPE, etc.). Some of the cited events did not result in personnel injuries, they were "near miss" events that did not cause injuries simply because no workers were nearby at the time of the event. All of these types of events, equipment failures, personnel judgments, and near misses, require closer examination for industrial safety. 
Table 5. Recent Power Reactor Events of Industrial Safety Concern

\begin{tabular}{|c|c|c|c|}
\hline $\begin{array}{l}\text { US NRC } \\
\text { Event } \\
\text { Notification } \\
\text { Report } \\
\text { number } \\
\end{array}$ & Plant & Date & Description of Event \\
\hline 35763 & Millstone & $05 / 25 / 1999$ & $\begin{array}{l}\text { The reactor was manually tripped from } 100 \% \text { power } \\
\text { because of a steam leak in the } 1 \text { A feedwater heater. } \\
\text { All control rods fully inserted. The plant is stable in } \\
\text { hot standby. The steam leak was discovered when } \\
\text { oscillations in the feedwater heater water level } \\
\text { resulted in a main control board annunicator. } \\
\text { Personnel sent to investigate the feedwater heater } \\
\text { reported a steam leak in the pipe leading up to the } \\
\text { flange to which the relief valve is connected. } \\
\text { Operators then manually tripped the reactor. Access } \\
\text { to the turbine building is restricted while the } \\
\text { feedwater heater is steaming down. There were no } \\
\text { injuries in this event. }\end{array}$ \\
\hline 36162 & Salem & $09 / 14 / 1999$ & $\begin{array}{l}\text { An employee was transported to Salem Hospital after } \\
\text { exhibiting heat stress symptoms after a containment } \\
\text { entry. A complete frisk could not be completed prior } \\
\text { to loading him in the ambulance. A radiation } \\
\text { technician was able to complete the frisk prior to the } \\
\text { arrival at the hospital, but after the hospital had been } \\
\text { mobilized to accept a contaminated, injured } \\
\text { individual. The frisk revealed that the individual was } \\
\text { not contaminated. The individual is expected to fully } \\
\text { recover. }\end{array}$ \\
\hline 36203 & $\begin{array}{l}\text { Three Mile } \\
\text { Island-1 }\end{array}$ & $09 / 21 / 1999$ & $\begin{array}{l}\text { During refueling operations, the licensee transported } \\
\text { a worker, who injured his lower back while working } \\
\text { inside containment on the upper manway, to the } \\
\text { Hershey Medical Center. He may have been } \\
\text { contaminated, so he was accompanied by Health } \\
\text { Physics personnel to the hospital. }\end{array}$ \\
\hline 32644 & Hope Creek & $09 / 29 / 1999$ & $\begin{array}{l}\text { At } 2117 \text { hours, the 'B' control room ventilation train } \\
\text { chiller tripped resulting in an automatic start of the } \\
\text { 'A' control room ventilation train. The trip was the } \\
\text { direct result of a major freon leak dispersing into the } \\
\text { auxiliary building. All non-essential personnel were } \\
\text { evacuated from the auxiliary building. An Unusual } \\
\text { Event was declared at } 2133 \text { hours in accordance with } \\
\text { Emergency Classification Guidance because the } \\
\text { release of toxic gas deemed detrimental to safe } \\
\text { operation of the plant. No personnel injuries have } \\
\text { occurred, and all access to the auxiliary building is } \\
\text { being controlled until follow-up actions are complete. } \\
\text { The leak was terminated because the full freon } \\
\text { charge in the chiller dispersed ( } 1 \text { metric ton, or } 2,200 \\
\text { pounds), and the atmosphere in the auxiliary building } \\
\text { has been restored to normal. The licensee terminated } \\
\text { the Unusual Event at } 0158 \text { hours on } 09 / 30 / 99 \text {. }\end{array}$ \\
\hline
\end{tabular}


Table 5. Continued.

\begin{tabular}{|c|c|c|c|}
\hline $\begin{array}{l}\text { US NRC } \\
\text { Event } \\
\text { Notification } \\
\text { Report } \\
\text { number }\end{array}$ & Plant & Date & Description of Event \\
\hline 36408 & Point Beach & $11 / 06 / 1999$ & $\begin{array}{l}\text { An employee slipped and fell in the plant laundry } \\
\text { room. An ambulance was called due to a possible } \\
\text { hip injury. The potentially contaminated person was } \\
\text { taken to a hospital. }\end{array}$ \\
\hline 36457 & $\begin{array}{l}\text { Brown's } \\
\text { Ferry-2 }\end{array}$ & $11 / 18 / 1999$ & $\begin{array}{l}\text { During replacement of an oil cooler discharge } \\
\text { temperature switch by Instrument Maintenance, the } \\
\text { pressure boundary of the oil system was } \\
\text { inadvertently breached for a total of approximately } \\
\text { five minutes (the work package assumed that the } \\
\text { Temperature Switch had a well, but it when the } \\
\text { Temperature Switch was removed it was discovered } \\
\text { that the switch did not have a well). The breach } \\
\text { occurred because adequate documentation was not } \\
\text { included in the work package. The switch was } \\
\text { successfully replaced. }\end{array}$ \\
\hline 36502 & Salem & $12 / 11 / 1999$ & $\begin{array}{l}\text { An instrument line on a } 10 \text { Ton Carbon Dioxide tank } \\
\text { broke off, causing the tank to discharge into the tank } \\
\text { storage area, which is located at the } 25.6 \mathrm{~m}(84 \text { foot) } \\
\text { level in the Auxiliary Building. No personnel were } \\
\text { in the area when the instrument line broke. Personnel } \\
\text { entered the room wearing self-contained breathing } \\
\text { apparatus and isolated the leak. No one was injured } \\
\text { by the incident. }\end{array}$ \\
\hline 36583 & River Bend & $01 / 12 / 2000$ & $\begin{array}{l}\text { At } 1515 \text { hours, an injured contract employee was } \\
\text { transported offsite to a hospital for medical treatment. } \\
\text { The individual was erecting scaffolding in the } \\
\text { Turbine Building, fell approximately } 6 \mathrm{~m} \text { ( } 20 \text { feet) } \\
\text { and sustained injuries. The individual was working } \\
\text { inside the Controlled Access Area, but not inside a } \\
\text { Contaminated Area, and a thorough survey for } \\
\text { radioactive contamination could not be performed } \\
\text { prior to transport. Therefore, the individual was } \\
\text { considered to be potentially contaminated. A } \\
\text { radiation protection technician accompanied the } \\
\text { ambulance to the hospital and a second radiation } \\
\text { protection technician has been dispatched to the } \\
\text { hospital as well. The injured contract employee has } \\
\text { been verified not contaminated. Preliminary medical } \\
\text { evaluation revealed no life threatening injuries. }\end{array}$ \\
\hline 36645 & $\begin{array}{l}\text { Quad Cities- } \\
2\end{array}$ & $01 / 31 / 2000$ & $\begin{array}{l}\text { At } 1005 \text { hours the control room received a report of a } \\
\text { small explosion in the Unit } 2 \text { pipeway between the } \\
\text { main steam isolation valve room and the low- } \\
\text { pressure heater bay. Control room personnel, in } \\
\text { accordance with station procedures, notified the local } \\
\text { fire department of an explosion and possible fire. } \\
\text { There were no personnel injuries, no equipment } \\
\text { damage, and no fire. As a result of the call to the fire } \\
\text { department, local law enforcement agencies and two }\end{array}$ \\
\hline
\end{tabular}


Table 5. Continued.

\begin{tabular}{|c|c|c|c|}
\hline $\begin{array}{c}\text { US NRC } \\
\text { Event } \\
\text { Notification } \\
\text { Report } \\
\text { number } \\
\end{array}$ & Plant & Date & Description of Event \\
\hline 36645 & $\begin{array}{l}\text { Quad Cities- } \\
2\end{array}$ & $01 / 31 / 2000$ & $\begin{array}{l}\text { (continued) television stations responded to the site. } \\
\text { For a period of time, the state road in the vicinity of } \\
\text { the plant was closed by law enforcement agencies. } \\
\text { The cause of the explosion reported to the control } \\
\text { room was a very loud, sudden noise that resulted } \\
\text { when a nylon bag containing small oxygen and } \\
\text { acetylene bottles and hoses was opened. The sound } \\
\text { was most likely the result of the ignition of a small } \\
\text { amount of oxygen and acetylene trapped in the bag. } \\
\text { Although no notification was made or planned for } \\
\text { local media or law enforcement support, personnel } \\
\text { from both of these areas were dispatched to the site. } \\
\text { There were no injuries or fire. No plant equipment } \\
\text { was affected. }\end{array}$ \\
\hline 37783 & Perry & $02 / 26 / 2000$ & $\begin{array}{l}\text { At } 1350 \text { hours, an injury occurred to a worker when } \\
\text { the individual fell and injured his back. At } 1426 \text {, the } \\
\text { worker was attended by local Emergency Medical } \\
\text { Personnel who transported the individual to the local } \\
\text { Hospital. Initial frisking of the individual showed no } \\
\text { contamination, but due to his back injury his back } \\
\text { was not completely monitored until arrival at the } \\
\text { hospital. At the hospital, it was determined the } \\
\text { individual had thee small (quarter size, i.e., } 2.5-\mathrm{cm} \\
\text { diameter) and very low level contaminated spots of } \\
100 \text { counts corrected above the background of } 80 \\
\text { counts per minute (cpm). One spot was located in } \\
\text { the back of the individual's head and was } \\
\text { immediately removed with water. Two additional } \\
\text { spots were detected on the worker's pants and these } \\
\text { were also immediately removed. The individual was } \\
\text { further surveyed and no contamination was found. } \\
\text { No dose was received by the individual as a result of } \\
\text { the contamination. The decon materials and clothing } \\
\text { were contained and returned to the plant by radiation } \\
\text { safety supervision. The ambulance, hospital } \\
\text { personnel and hospital materials were all surveyed } \\
\text { and released as no contamination was detected. }\end{array}$ \\
\hline 36813 & Catawba-2 & $03 / 20 / 2000$ & $\begin{array}{l}\text { A vendor support employee was injured due to a fall } \\
\text { (approximately } 1.5 \text { to } 2.4 \mathrm{~m} \text {, or } 6 \text { to } 8 \text { feet) in the } \\
\text { lower containment. The individual was supporting } \\
\text { reactor coolant pump work. Due to injuries, the } \\
\text { individual could not be fully frisked for } \\
\text { contamination. Until further determination, } \\
\text { individual is considered potentially contaminated and } \\
\text { is being transported offsite to the local medical } \\
\text { facility. Further investigation revealed that the }\end{array}$ \\
\hline
\end{tabular}


Table 5. Continued

\begin{tabular}{|c|c|c|c|}
\hline $\begin{array}{c}\text { US NRC } \\
\text { Event } \\
\text { Notification } \\
\text { Report } \\
\text { number } \\
\end{array}$ & Plant & Date & Description of Event \\
\hline 36813 & Catawba-2 & $03 / 20 / 2000$ & $\begin{array}{l}\text { (continued) injured individual was not contaminated, } \\
\text { however the harness and nylon choker used to lift the } \\
\text { injured man has some low level fixed contamination. } \\
\text { These materials have been transported back to the } \\
\text { site and all areas of the hospital and the transport } \\
\text { vehicle have been surveyed clean. }\end{array}$ \\
\hline 38369 & $\begin{array}{l}\text { Crystal } \\
\text { River-3 }\end{array}$ & $10 / 09 / 2001$ & $\begin{array}{l}\text { During refueling, a contract worker injured his leg } \\
\text { while working in the Reactor Building. The injury } \\
\text { required the worker to be transported offsite to a } \\
\text { local medical facility. A Health Physics technician } \\
\text { (HP) accompanied the individual to the medical } \\
\text { facility since he was potentially contaminated. } \\
\text { Subsequent survey found radioactive contamination } \\
\text { on the individual's clothing and on the backboard } \\
\text { used for transport. The HP took custody of the } \\
\text { contaminated material and returned it to the plant. }\end{array}$ \\
\hline 38822 & $\begin{array}{l}\text { Nine Mile } \\
\text { Point }\end{array}$ & $04 / 02 / 2002$ & $\begin{array}{l}\text { The licensee declared an unusual event at } 0029 \text { hours } \\
\text { due to a } \mathrm{CO}_{2} \text { discharge from a broken hose reel on } \\
\text { the turbine building fire suppression } \mathrm{CO}_{2} \text { system. } \\
\text { The hose reel was broken during work activities. The } \\
\text { turbine building, reactor building, radioactive waste } \\
\text { building, and control building were evacuated. The } \\
\text { plant's control room was not evacuated. The broken } \\
\text { hose reel was isolated to terminate the release of } \\
\mathrm{CO}_{2} \text {. No personnel injuries were identified as a } \\
\text { result of the event. }\end{array}$ \\
\hline 38959 & $\begin{array}{l}\text { Peach } \\
\text { Bottom }\end{array}$ & $06 / 02 / 2002$ & $\begin{array}{l}\text { The "Cardox system" fire suppression system } \\
\text { injected } \mathrm{CO}_{2} \text { in the emergency diesel room at } 0033 \\
\text { hours, during diesel testing. An alert was declared at } \\
0102 \text { due to the detection of toxic } \mathrm{CO}_{2} \text { gas within a } \\
\text { vital area. The room was evacuated safely (only two } \\
\text { people were in the room at that time) and there were } \\
\text { no injuries. There was no fire damage and the } \mathrm{CO}_{2} \\
\text { tank was isolated. }\end{array}$ \\
\hline 39180 & Wolf Creek & $09 / 10 / 2002$ & $\begin{array}{l}\text { At approximately } 1400 \text { hours a report was received in } \\
\text { the control room of a non-work related medical } \\
\text { emergency involving a contract employee. Onsite } \\
\text { medical personnel responded and commenced first } \\
\text { aid including cardiopulmonary resuscitation. An } \\
\text { ambulance was dispatched and the individual was } \\
\text { transported offsite to a local hospital. Station } \\
\text { personnel were subsequently notified by hospital } \\
\text { personnel that the contract employee had passed } \\
\text { away. }\end{array}$ \\
\hline
\end{tabular}


Table 5. Continued.

\begin{tabular}{|c|c|c|c|}
\hline $\begin{array}{c}\text { US NRC } \\
\text { Event } \\
\text { Notification } \\
\text { Report } \\
\text { number }\end{array}$ & Plant & Date & Description of Event \\
\hline 39269 & St. Lucie & $10 / 11 / 2002$ & $\begin{array}{l}\text { At } 0925 \text { hours, a release of chlorine gas was noted } \\
\text { from the sodium hypochlorite area, and smell of } \\
\text { chlorine gas was noted in the Turbine Generator } \\
\text { Building. All personnel were evacuated from the } \\
\text { Turbine Generator Building as a precautionary } \\
\text { measure, and emergency personnel were dispatched } \\
\text { to determine the concentration of chlorine gas. At } \\
\text { the release site the chlorine gas concentration was } 3 \\
\text { ppm. Per the material safety data sheet, the } \\
\text { Immediately Dangerous to Life or Health limit is } 30 \\
\text { ppm. No Emergency Action Levels were entered. } \\
\text { Additional samples were taken in the Turbine } \\
\text { Generator building prior to allowing unrestricted } \\
\text { access into the area. Both Units' Control Rooms } \\
\text { were placed in the air recirculation mode as a } \\
\text { precautionary measure. There were no injuries from } \\
\text { this event. The leak was stopped. The chlorine gas } \\
\text { was generated while cleaning the NaOCl lines with } \\
\text { muriatic acid to remove calcium buildup. The acid } \\
\text { caused evolution of gaseous chlorine. The licensee } \\
\text { investigated why chlorine gas was released this time } \\
\text { when it was not released when the lines were cleaned } \\
\text { in the past. The licensee surmised that they may } \\
\text { have used a stronger concentration of muriatic acid } \\
\text { this time. (note: NaOCl is used for chlorination to } \\
\text { prevent biofouling in heat exchange equipment). }\end{array}$ \\
\hline 39321 & $\begin{array}{l}\text { Arkansas } \\
\text { Nuclear-1 }\end{array}$ & $10 / 24 / 2002$ & $\begin{array}{l}\text { While working under the Reactor Vessel Head, an } \\
\text { individual bumped his head on a Control Rod Drive } \\
\text { Lead Screw. The contact resulted in a } 2.5-\mathrm{cm} \\
\text { laceration on the side of his head. The Emergency } \\
\text { Medical Response Team was activated and } \\
\text { responded to the scene. The worker was conscious } \\
\text { but dazed. An initial survey for contamination was } \\
\text { performed but was inconclusive due to background } \\
\text { levels at the scene and due to a head brace that had } \\
\text { been placed on the individual as a precaution to } \\
\text { guard against neck injury. An ambulance was called } \\
\text { to the site and the individual was transported with a } \\
\text { Health Physicist to the regional Medical Center. } \\
\text { Additional surveying of the individual revealed that } \\
\text { the individual's head was slightly contaminated at } \\
\text { 100 cpm above background and the backboard used } \\
\text { in transport had } 150 \text { cpm. The individual was } \\
\text { decontaminated by plant Health Physics personnel } \\
\text { dispatched to the hospital. All of the radioactive } \\
\text { material was retrieved and returned to the site. The } \\
\text { individual is in satisfactory condition and the wound } \\
\text { required three stitches to close. }\end{array}$ \\
\hline
\end{tabular}


Table 5. Continued

\begin{tabular}{|c|c|c|c|}
\hline $\begin{array}{l}\text { US NRC } \\
\text { Event } \\
\text { Notification } \\
\text { Report } \\
\text { number } \\
\end{array}$ & Plant & Date & Description of Event \\
\hline 39513 & Cook-1 & $01 / 15 / 2003$ & $\begin{array}{l}\text { At } 2010 \text { hours, a fault occurred in the Unit } 1 \text { Main } \\
\text { Transformer, resulting in a fire. The fault caused an } \\
\text { automatic main generator trip and an immediate } \\
\text { turbine trip and reactor trip. The fire was originally } \\
\text { extinguished at } 2035 \text {, with one minor reflash that was } \\
\text { promptly controlled by the fire brigade. The } \\
\text { Emergency Plan was activated at the Unusual Event } \\
\text { level due to a fire within the protected area not being } \\
\text { extinguished within fifteen minutes. All applicable } \\
\text { notifications were made for the Emergency Plan } \\
\text { declaration, including a one-hour report to the NRC } \\
\text { at } 2100 \text { hours. There was one minor personnel injury } \\
\text { involved that required off-site medical attention, due } \\
\text { to the person suffering a fall and smoke inhalation. }\end{array}$ \\
\hline 39596 & Callaway & $02 / 13 / 2003$ & $\begin{array}{l}\text { At } 0954 \text { hours the Control Room was notified of a } \\
\text { personnel injury in the Turbine building. After } \\
\text { examination by the site doctor, the individual was } \\
\text { transported off site for treatment. Subsequently, on } \\
\text { February } 18,2003 \text {, the individual was admitted to the } \\
\text { hospital for further treatment. Preliminary } \\
\text { investigation indicates that the individual was struck } \\
\text { in the face with a flying object. The individual was } \\
\text { using a filter change out tool and attempting to } \\
\text { disconnect a } 5 \mathrm{~cm} \text { Camflex plug. The line was } \\
\text { apparently pressurized, resulting in plug ejection } \\
\text { toward the individual's face after it was disconnected. }\end{array}$ \\
\hline 39695 & Susquehanna & $03 / 24 / 2003$ & $\begin{array}{l}\text { At } 1652 \text { hours, the plant declared an Unusual Event } \\
\text { for a contaminated individual transported offsite. } \\
\text { The individual had fallen while working in the } \\
\text { drywell of the Primary Containment. The plant is } \\
\text { presently in a refuel outage. First Aid personnel were } \\
\text { dispatched and treated her injuries. The individual's } \\
\text { injuries prevented the removal of all of the protective } \\
\text { clothing. Based on the inability to remove the } \\
\text { clothing and completely frisk the individual, the } \\
\text { individual is being considered contaminated. At } \\
1657 \text { hours a local ambulance transported the worker } \\
\text { offsite en route to a local hospital. }\end{array}$ \\
\hline 40157 & $\begin{array}{l}\text { Fort } \\
\text { Calhoun }\end{array}$ & $09 / 14 / 2003$ & $\begin{array}{l}\text { At about } 0549 \text { hours, a slightly contaminated worker } \\
\text { who was exhibiting heat stress symptoms was } \\
\text { transported to an off-site medical facility for } \\
\text { treatment. The worker was very slightly con- } \\
\text { taminated on the forearms and elbows. The worker } \\
\text { was treated and is being observed prior to being } \\
\text { released. No contamination was spread off-site. }\end{array}$ \\
\hline
\end{tabular}


Table 5. Continued.

\begin{tabular}{|c|c|c|c|}
\hline $\begin{array}{c}\text { US NRC } \\
\text { Event } \\
\text { Notification } \\
\text { Report } \\
\text { number }\end{array}$ & Plant & Date & Description of Event \\
\hline $\begin{array}{l}\text { Morning } \\
\text { report, } \\
\text { 4-2003-0016 }\end{array}$ & $\begin{array}{l}\text { South Texas } \\
\text { Project }\end{array}$ & $12 / 09 / 2003$ & $\begin{array}{l}\text { The licensee was performing a surveillance test run } \\
\text { of emergency diesel generator } 22 \text {. The diesel } \\
\text { generator had been operating at full rated load for } \\
\text { approximately } 20 \text { minutes when the control room } \\
\text { received alarms indicating diesel generator trouble } \\
\text { and that the diesel generator output breaker was } \\
\text { open. In response, an operator shutdown the engine } \\
\text { for the generator by pulling the engine fuel rack } \\
\text { shutoff lever. Initial inspection by the licensee } \\
\text { revealed that parts of the generator's, number } 9 \mathrm{R} \\
\text { piston and connecting rod, had been ejected from the } \\
\text { engine. The ejected parts created a large hole in the } \\
\text { southeast side of the crankcase. There were no } \\
\text { personnel injuries. The licensee is evaluating the } \\
\text { damage and has formed a team to determine the } \\
\text { cause of the failure. }\end{array}$ \\
\hline 40580 & Columbia & $03 / 10 / 2004$ & $\begin{array}{l}\text { This notification is being made to report an onsite } \\
\text { fatality due to a non-work related, personal medical } \\
\text { condition. During the incident, plant First Responder } \\
\text { personnel responded to a Control Room notification } \\
\text { of a man down. First Responder personnel initiated } \\
\text { cardiopulmonary resuscitation and used an } \\
\text { Automated External Defibrillator and continued until } \\
\text { the Hanford Fire Department ambulance paramedics } \\
\text { responded. The individual was taken via ambulance } \\
\text { to Kadlec Medical Center in Richland, Washington } \\
\text { where the individual was pronounced dead. The } \\
\text { fatality occurred in the Protected Area and was not } \\
\text { associated with any work inside a radiological area. }\end{array}$ \\
\hline 40677 & Oconee-2 & $04 / 16 / 2004$ & $\begin{array}{l}\text { A vendor received a possible ankle fracture while } \\
\text { working in the Reactor Building. The injured person } \\
\text { was descending a ladder and stepped on a roll of poly } \\
\text { material and injured his ankle when the roll shifted. } \\
\text { The onsite medical assistance team responded and } \\
\text { removed the vendor from the reactor building and } \\
\text { prepared him for transport to a hospital using a } \\
\text { hospital ambulance. Due to the nature of the injury, } \\
\text { he was unable to stand in the whole body frisker and } \\
\text { was transported to the hospital as potentially } \\
\text { contaminated. A Radiation Protection Technician } \\
\text { accompanied the injured person to the hospital where } \\
\text { it was determined that he was not contaminated. }\end{array}$ \\
\hline
\end{tabular}




\section{Chapter 4 References}

Bangs, 1986. S. Bangs, "When a weld fails...", Welding Design \& Fabrication, March 1986, pages 79-82.

Bertini, 1980. H. W. Bertini, Descriptions of Selected Accidents that Have Occurred at Nuclear Reactor Facilities, ORNL/NSIC-176, Oak Ridge National Laboratory, April 1980.

BLS, 1967. Work Injury Experience in Atomic Energy 1965 and 1966, BLS Report 334, PB 227 527, Bureau of Labor Statistics, Washington DC, 1967.

BLS, 1969. Work Injuries in Atomic Energy, 1967, BLS Report 359, PB 227524 , Bureau of Labor Statistics, Washington DC, December 1969.

BLS, 1970. Work Injuries in Atomic Energy, 1968, BLS Report 378, PB 227 540, Bureau of Labor Statistics, Washington DC, 1970.

BLS, 1971. Work Injuries in Atomic Energy, 1969, BLS Report 385, PB 227 528, Bureau of Labor Statistics, Washington DC, 1971.

BLS, 1972. Work Injuries in Atomic Energy, 1970, BLS Report 411, Bureau of Labor Statistics, Washington DC, 1972.

Burrows, 2002. S. Burrows and D. A. Hagemeyer, Occupational Radiation Exposure at Commercial Nuclear Power Reactors and Other Facilities, 2001, thirty-fourth annual report, NUREG-0713, Volume 23, US Nuclear Regulatory Commission, Washington DC, September 2002.

Cadwallader, 1999. L. C. Cadwallader and D. A. Petti, "Review of availability growth in energy production technologies," Proceedings of the $18^{\text {th }}$ IEEE/NPSS Symposium on Fusion Engineering (SOFE 99), October 25-29, 1999, Albuquerque, New Mexico, IEEE (1999) 585-588.

Cochran, 1999. R. G. Cochran and N. Tsoulfanidis, The Nuclear Fuel Cycle: Analysis and Management, second edition, American Nuclear Society, La Grange Park, IL, 1999 , chapter 11.

ENR, 2002. US NRC Event Notification Report, Offsite Notification at IP2 of Onsite Fatality, power reactor event notification report 39074, July 19, 2002, available at the web site nrc.gov.

INPO, 2003. 2002 Performance Indicators for the U.S. Nuclear Industry, Institute of Nuclear Power Operations, Atlanta, GA, 2003, available at the web site inpo.org. 
LER, 2002. "138 kV Ground Protection Trip Results in Auto Start of Emergency Diesel Generators," Licensee Event Report 2002-003, docket number 05000247, October 11, 2002.

Loomis, 1999. D. Loomis, V. Dufort, R. C. Kleckner, and D. A. Savitz, "Fatal Occupational Injuries Among Electric Power Company Workers," American Journal of Industrial Medicine, 35 (1999) 302-309.

NN, 2001. "Statistics show U.S. Nuclear plants always improving," Nuclear News, 44 (May 2001) 38-40.

NRC, 1982. Failures in Turbine Exhaust Lines, Information Notice 82-22, US Nuclear Regulatory Commission, July 9, 1982.

NRC, 1986. Feedwater Line Break, Information Notice 86-106, US Nuclear Regulatory Commission, December 16, 1986.

NRC, 1988. Electrical Shock Fatalities at Nuclear Power Plants, Information Notice 8896, US Nuclear Regulatory Commission, December 14, 1988.

NRC, 1993. Bursting of High Pressure Coolant Injection Steam Line Rupture Discs Injures Plant Personnel, Information Notice 93-67, US Nuclear Regulatory Commission, August 16, 1993.

O'Donnell, 1981. F. R. O'Donnell and H. C. Hoy, Occupational Safety Data and Casualty Rates for the Uranium Fuel Cycle, ORNL-5797, Oak Ridge National Laboratory, December 1981.

Rippon, 1981. S. Rippon, "Comparison of comparative risks," Nuclear News, 24 (February 1981) 58-60.

Sailor, 1985. V. L. Sailor and J. J. Colbert, $\underline{\text { Summary of Barrier Degradation Events and }}$ Small Accidents in U.S. Commercial Nuclear Power Plants, NUREG/CR-4067, US Nuclear Regulatory Commission, March 1985.

WANO, 2003. 2003 Performance Indicators, World Association of Nuclear Operators, London, UK, June 2004, available at the web site wano.org.

Yager, 2001. J. W. Yager, M. A. Kelsh, Ke Zhao, and R. Mrad, "Development of an Occupational Illness and Injury Surveillance Database for the Electric Energy Sector," Applied Occupational and Environmental Hygiene, 16 (2001) 291-294. 


\section{CONCLUSIONS AND RECOMMENDATIONS}

These data provide several insights. The fusion experiment data have shown that the expectation of any accident events being directly related to the unique aspects of fusion experiments (much use of electrical power, cryogens, vacuum reservoir, radiofrequency heating, compressed gases, etc.) is not strictly true. Many of the occupational accidents described in this report are related to typical industrial safety concerns, such as inadvertent power up of equipment, falling from height, dropped crane loads, trench wall collapse, and other events. Some of the events did involve electrical shock, oxygen deficiency, and radiation overexposure, which are more directly related to fusion but can also occur in some other industrial activities. In an official auditing activity and in subsequent reporting, it was found that fusion worker compliance with occupational safety and health rules, especially lockout-tagout electrical safety and confined space safety, was not consistent. The fusion events do serve to indicate what areas of occupational safety are important and should be examined for ITER.

The first insight for the particle accelerator facilities is that these facilities are not as benign as is generally believed in the nuclear industry. Because these facilities do not handle large thermal energies in the form of superheated steam or high temperature, high pressure liquids, and the radiation created at the accelerator's target is well shielded with concrete and earth, many believe that accelerators pose a very low hazard to workers. While there have not been any occupational fatalities during operation or maintenance of these machines, there have been some severe occupational injuries, particularly electrical injuries. This report has also documented that there have been construction fatalities at accelerators, meaning that we should endeavor to guard against construction fatalities during ITER construction since ITER is more complex than most accelerators. Accelerator radiation safety reports have shown that ionizing and non-ionizing radiation safety has not always been practiced; some inadvertent exposures have occurred. Another insight is the trend for the past $~ 10$ years of hiring small companies to perform tasks in the facilities on an 'as needed' basis. This practice reduces operating costs by reducing the number of permanent staff and having maintenance and construction teams on site only when needed for new projects. Such contracting is also thought to increase machine availability because larger numbers of outside workers can be allocated to tasks during facility outages so that outage time is decreased. If a smaller, permanent work force is used, outages tend to be longer duration. These outside companies send skilled, licensed craftsmen, but the craftsmen are not familiar with the facility and appear to not be well supervised by accelerator personnel. Some of these subcontract workers have suffered severe injuries at the accelerators, and some injuries have also occurred at fusion experiments. Occupational safety specialists refer to such situations as "setting the person up to fail", which means placing a worker into an industrial environment with insufficient knowledge and supervision so that he is highly likely to make an error that will either harm himself or others, or both. From an occupational safety perspective, ITER should not be operated and maintained by subcontracted companies unless the contracts are for long terms (e.g., 10 years or more), which gives the staff of the subcontracted company the time to become familiar with the facility and its operation. The accelerators have continued to operate with these occupational injuries; however, the 
evidence from other industries is that safe operations offer higher efficiency and productivity. With ITER being the most expensive machine of its type ever built and being a showcase for worldwide fusion, safe operations that improve machine performance are highly desirable.

The fusion experiment and accelerator occupational safety data showed that technicians are the most frequently injured workers in these facilities. This is due to the fact that the technicians are most often working 'hands-on' with the components and systems of the experiment and suffer the minor injuries (contusions, lacerations, sprains, etc.). Some of the occupational accidents described in this report were caused by workers not following the existing safety rules. Occupational safety specialists are quick to point out that OSHA rules are minimums that positively affect worker safety; if the workers follow even only these minimum OSHA regulations then they have increased their protection and reduced losses. Other occupational accident events in this report were created by workers not realizing all the issues or hazards of the workplace, such as the incident where an inadvertent movement of an overhead crane allowed the crane hook to strike and dislodge a hand rail, which fell from height and struck a worker. A third category of accident noted in this report is workers exposed to energies or substances released when equipment has failed. Fusion experiments and the accelerators had few accidents of this latter category, but the brief review of power plant data revealed a number of accidents originating with breached or failed equipment. Since ITER lies somewhere between present day fusion experiments and power plants, the human decisions and awareness aspect of accidents, and the equipment failure aspect of accidents, must both be addressed for occupational safety.

The nuclear fission plant occupational safety data did not have very fine resolution. The Bureau of Labor Statistics does not divide the power plant worker data by type of plant or by occupation within the electric power industry. Therefore, it is difficult to determine if any one occupational type (e.g., electrician, welder, mechanic, etc.) has had the highest injuries or if one occupation was at higher risk than another. The power plant data had fatality frequency estimates while there have not been any operational fatalities at the fusion experiments or accelerators. However, the power plant worker accident rates were lower than the comparable lost work case rates for both fusion and accelerators, this may be due to the hands-on nature of experiments that are often shut down for modifications and other reasons while high availability power plants have less work performed during operation. There are probably differences in the consequences of the injuries; the DOE data is typically low consequence, but the power plant worker accident consequence information is not known. The fission power plant data can be used in goal-setting for a robust fusion experiment that is similar to a power plant, but the fission power data are not otherwise very helpful except as case histories. Reasons for the lack of resolution in the data are not clear. It is suspected that since the radiological aspects of nuclear power are often exaggerated by the media, there is reluctance on the part of public utilities to discuss any type of accident event in a nuclear plant that is not mandated by law.

The event descriptions provided here can serve to support occupational safety analysis for the ITER design. A room-by-room analysis of ITER for occupational hazards is planned. 